UC-NRLF

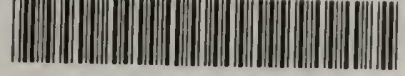

B 4 619 896 


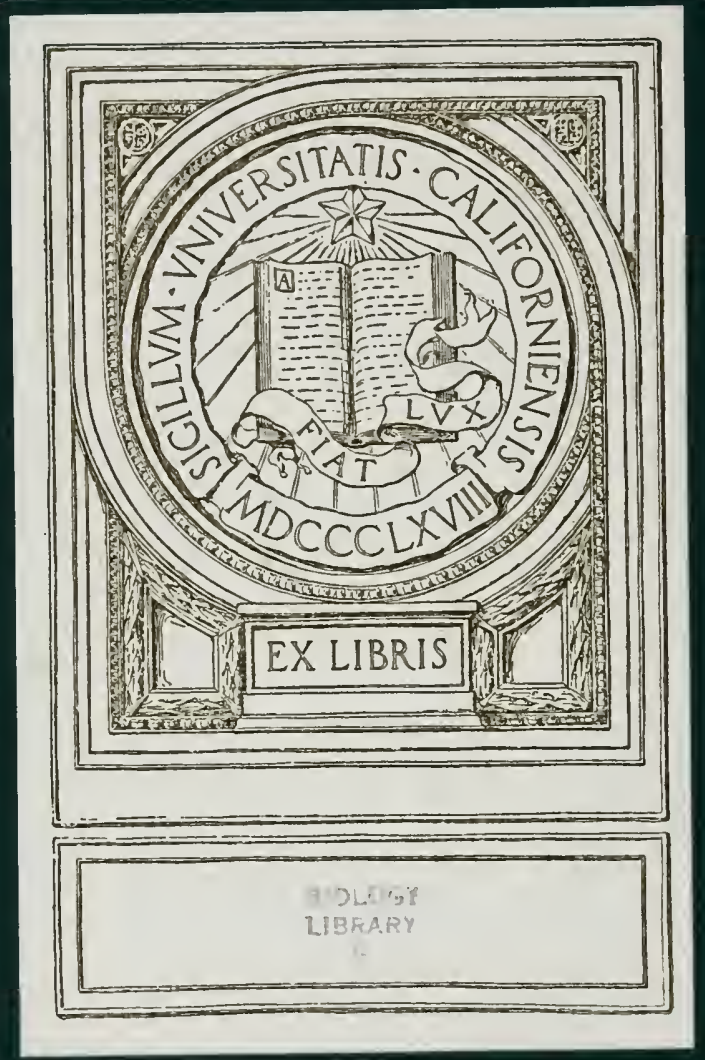





THE

\section{ETIOLOGY AND PATHOLOGY}

OF

GROUSE DISEASE \& FOWL ENTERITIS 

THE

\title{
ETIOLOGY AND PATHOLOGY
}

\author{
OF
}

\section{GROUSE DISEASE FOWL ENTERITIS}

And some other Diseases affecting Birds

E. KLEIN, M.D., F.R.S.

LECTURER OF GENERAL ANATOMY AND PHYSIOLOGY IN THE MEDICAL SCHOOL, OF ST. BARTHOLOMEW'S HOSPITAL, LONDON

IVITH FIFTY-THREE ILLUSTRATIONS

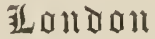

MACMILLAN AND CO.

AND NEIV YORK

I 892 


$$
\begin{aligned}
& \text { SF } 995 \\
& K 6
\end{aligned}
$$


SIR JOSEPH LISTER, BART., M.D., LL.D., F.R.S., THIS BOOK

IS RESPECTFULLY DEDICATED

BY

THE AUTHOR 



\section{P R E F A C E}

HAVING been engaged for the last five years in collecting data as to the etiology and pathology of grouse disease, I find that the time has arrived when I am enabled to give a coherent account of the subject; the number of grouse, dead or dying naturally from the disease, which I have dissected, and which were derived from different parts of England and Scotland, sent to me during several years through the kindness of the Editor of the Field, is very considerable, as will be fully referred to later; the number of cultivations from the grouse, the number of experiments with cultivations on birds and manmals has been sufficiently large to enable me to draw definite conclusions as to the nature of those cultivations. To the Editor of the Field, and through him to many gentlemen, both in England and Scotland, interested in grouse, my best thanks are due for the valuable assistance which I received from them. The second disease treated here is a 
highly infectious and fatal disease of fowls-fowl enteritis, which was at first believed to be fowl cholera, to which it bears a certain resemblance, but from which it materially differs, not so much as to its mortality and high degree of infectiousness, but in respect to its pathology and causation. In order to show these differences I have prefaced the chapters on fowl enteritis with a short chapter on fowl cholera. The first material of fowl enteritis on which I worked was placed at my disposal by Mr. W. Cook of Orpington, the well-known author on, and successful breeder of poultry, through the intervention of Mr. C. Wellington; both these gentlemen, notably Mr. Cook, have rendered me invaluable service, for which I beg to return them my best thanks.

The third disease, about which I intend to make a few remarks, is the infectious disease of young pheasants known to pheasant-breeders as "the cramps." While engaged in Ayrshire for the Field, in the summer of 1887 , in inquiring into the pathology and etiology of the grouse disease, I had the opportunity of studying the cramps at Blairquhan, and to the keeper there, Mr. Douglas, my thanks are due for supplying me then and afterwards with materials both as living and dead specimens. 
In conclusion I wish to thank my friend $\mathrm{Mr}$. Andrew Pringle for having kindly prepared many of the micro-photograms, illustrating the microscopic appearances, both of the grouse disease and of the fowl cholera and fowl enteritis, and to my friend and pupil, Mr. E. C. Bousfield, for some of the micro-photograms and of the photograms of the cultivations.

ST. Bartholomew's Hospital,

January I 892 . 



\title{
CONTENTS
}

\author{
P A R T I
}

THE GROUSE DISEASE

CHAPTER I

Nature of the Disease . . . . . . . . I

PAGE

CHAPTER II

Symptoms and Pathology . . . . . . 13

CHAPTER III

Character of the Bacillus of the Grouse Disease . 24

CHAPTER IV

Experinents with Culture by Subcutaneous Inocula-

TION . . . . . . . 33

CHAPTER V

The Autumal Disease . . . . . . 44

CHAPTER VI

Further Characters of the Bacillus . . . . 5 I

CHAPTER VII

General Considerations . . . . . 58 


\section{CHAPTER VIII}

PAGE

a Pathogenic Bacillus resembling the Bacillus of the Grouse Disease . . . . . . . . 63

\section{P A R T II}

FOWL CHOLERA, FOWL ENTERITIS, AND "CRAMPS"

IN YOUNG PHEASANTS

CHAPTER IX

Fowl Cholera . . . . . . . . 79

CHAPTER X

Fowl Enteritis-Nature of the Disease . . . 86

CHAPTER XI

Cultivation of the Bacillus of Fowl Enteritis . 92

CHAPTER XII

EXPERIMENTS ON FowLS

CHAPTER XIII

Attenuation of the Virus of Fowl Enteritis . . 107

CHAPTER XIV

"Cramps" in young Pheasants . . . . . I 2 I

Figures 1 TO 53 . . . . 


\section{PART I}

\section{THE GROUSE DISEASE}





\section{CHAPTER I}

NATURE OF TIE DISEASE

THE epidemic disease which affects Red Grouse (Lagopus scoticus) in Scotland, the North of England, Wales, and Ireland during the spring and the summer, and which, particularly during April, May, and June of some years, makes such sad havoc amongst them, is the one which goes under the name of the Grouse Disease. Not that grouse and allied species are exempt from other affections, but the disease which for years past, in one or another part of the north of the British Isles, has carried away annually large numbers of these birds, is the one known as the "Grouse Disease." Nor do we mean that this disease occurs amongst the red grouse in spring and summer-time only, for there is good evidence to show that though these are the seasons in which the plague, when occurring, is most conspicuous owing to its extensive mortality, the autumn and winter are not free 
from the presence of the malady, which owing to various circumstances to be mentioned hereafter is then less conspicuous, and may even be altogether overlooked.

The fact that year after year in one or another part of the country the disease is or becomes epidemic, is in itself strong primâ facie evidence that it is an infectious illness; further, the now wellrecognised occurrence of isolated fatal cases of the same illness amongst the grouse in one or another part of a given district during autumn and winter, makes it certain that the disease has become endemic, and is ready, when favouring opportunities offer themselves, to assume the epidemic character. This is the case with some other acute infectious endemic maladies affecting men and animals which, at certain times of the year and under certain other favouring conditions, assume the epidemic character. Such a change is, however, a slow and gradual development. Take, for instance, Asiatic cholera, endemic in Bengal. It is present in isolated cases all the year round in some part or another of Bengal.' During the cold season the number of cases gradually increases; by the end of the cold season, during the spring and beginning of summer, the cases become so numerous, and the spread of the disease so conspicuous, even outside the endemic area, that the disease possesses 
the epidemic character; then, with the commencement of the rainy season, it gradually diminishes again, and reaches its lowest level towards the end of this and the commencement of the cold weather. This same character is observed in countries in which cholera is not endemic, and which remain free from it for years, e.g. Syria, Egypt, and European States. Cholera imported into these areas gradually and steadily works itself up into an epidemic, and equally gradually again declines, the summer and spring being the most favourable seasons in which it thus becomes epidemic. During autumn, and towards the winter, the cases become less numerous and milder, and as such may occur as isolated cases all through the winter. Towards spring they become again more numerous and more severe, and in spring may cause a recrudescence of the epidemic. Scarlet fever, diphtheria, and typhoid fever are other well-known instances in which acute endemic infectious disorders during certain seasons show marked exacerbations. In grouse disease the month of April, and the commencement of May, are the times in which the disease, as a rule, gradually by numbers and intensity (fatal cases) becomes conspicuous. During the end of May and the commencement of June it is at its height, and then again declines during July; but fatal cases occur also during August, September, 
October, and later, but their numbers are, for obvious reasons, smaller during this time. That a moor, or even a whole district, is and remains free from the epidemic during one or more years may be, and as a rule is, due to a variety of conditions which also as regards epidemics of other infectious diseases are known to occur; some of these we do know and call secondary or accessory conditions. Assuming the disease to be an infectious disease, there is nothing extraordinary in the fact, occasionally observed, and used by some as an argument against the infectious nature of the malady, to wit, that in a given country a series of moors are seriously implicated, while here and there one or another moor is only slightly affected, or apparently remains free from the disease. I say there is nothing extraordinary in this because there may be, for all we know, a variety of circumstances at work which favour, or disfavour, the spread of infection from one moor to the neighbouring moor. In human epidemics this fact is observed repeatedly. One locality becomes a hot-bed for the disease; the neighbouring locality remains fairly free from disease, or has only few cases. In a third locality at a greater distance the disease is again rife, and so on. Whether, in the case of the grouse disease, birds are in better condition to resist the disease in one locality than in the neighbouring one; 
whether birds affected with the disease carry it from one locality, not to the one lying next, but to a moor some distance off; whether in one moor there are present conditions as to situation, heather, water, etc., which are favourable or unfavourable to the development and spread of the disease,-all these are points which may, and probably (see below) do, play a part in the dissemination of the plague.

A disease which year after year assumes the character of an epidemic at particular seasons, which commences as isolated cases, and gradually works itself up into a serious epidemic, i.e. spreads from a few to many individuals, and again in the same way gradually declines; further, a disease which has such uniform and typical characters, both as to symptoms and pathology; bears on the face of it the character of an acute infectious disease.

The late Dr. Cobbold, in his well-known brochure on the grouse disease, enunciated that the cause of the grouse disease is a parasite, in fact a nematoid worm belonging to the group of strongyles (Strongylus pergracilis), for in the intestines of grouse dead of the disease this parasite was constantly present. Numerous grouse which he examined, and which succumbed either in the epidemic of 1872 or I873, and others examined in later years, contained these parasites in abundance, though "without doubt" 
(Cobbold, Parasites, p. 439) "the occasional presence of numerous tape-worms (Tania calva) hastened the consequent fatality." This view has always been strenuously opposed by various writers and observers, both lay and veterinary or medical, inasmuch as it was considered quite insufficient to account for the disease; for it was pointed out by Dr. Farquharson and Mr. Wilson (see below), and can be easily verified, that the same parasites occur also in birds not subject to the disease. Now, taking into consideration that the disease is well characterised both in its symptoms and its pathology; that the same parasites undoubtedly occur in grouse not afflicted with the grouse disease; that grouse dead of the disease are found sometimes plump, sometimes emaciated; and, above all, that grouse dead of the disease, in several instances when examined, showed no presence of the strongylus: it must be evident that the parasites sometimes present in the intestine cannot be the real cause, the cansa causans, of the disease. That cause must be of a different nature. It must be of a nature which works rapidly, or fairly rapidly, which affects the system as a whole, and which easily spreads amongst the birds. We shall presently see that one of the most prominent pathological changes in the diseased grouse is an acute congestion of one or both lungs, and that this change, whether very severe or less 
severe, is independent of the presence of strongylus. I dissected numerous birds dying or dead of the disease in 1887 , and showing the characteristic lung change, and must confess that I was extremely surprised to find the strongylus in some cases entirely absent, in others present in very small numbers, though tænia calva was present in all in large numbers, just as large as in birds killed but not affected with the grouse disease.

Amongst various writers on the grouse disease, none deserve a higher place than Dr. H. Colquhoun and Dr. D. G. F. Macdonald. By selecting the opinions of these two writers amongst a host of writers who, by letters to the Times and the Field or other publications, have contributed to this question, I am practically quoting in a condensed form the most noteworthy summary of the various opinions offered. All these opinions as to the cause of grouse disease do not in reality touch the causa causans at all, but the conditions or secondary causes, which favour, and play an important part in, the appearance of the grouse disease in the epidemic form. Amongst these conditions may be mentioned:- $(a)$ bad season; (b) bad or insufficient food; and (c) overstocking. It must be obvious that as in many other acute infectious disorders amongst men or animals these conditions play a very important part, inasmuch as 
they create a less resisting, more susceptible material for the carrying out of infection. The more adverse the season-inclemency of the weather, frost, etc.the more sparse or inferior the natural food, the less hardy and strong are the birds when the time arrives which is most favourable for the development of the disease, and therefore the more easily do they take infection. This is well understood as applicable to other infectious disorders. A strong individual, not weakened by bad and insufficient food or by bad or inclement weather, will resist the infection much more easily than one previously brought down in general health and strength. Suppose there are, on a given moor, a large number of birds in this weakened state, and suppose there exists on this moor the active cause of the disease, it stands to reason that, there being present a good many birds of no great resisting power, one or the other of them will take the infection, from these others will take it, and so on, so that, after a short time, a large number of foci will thus be started acting not only on the remainder of the weaker but also on the stronger birds. But supposing, on the other hand, the birds in early spring are well and strong; though the active cause of the disease be present on this moor, yet it will not easily find, as it were, a susceptible individual, and consequently the disease will show itself either only in 
sporadic cases, or will be barely appreciable, or will be absent altogether.

And so also with overstocking and over-preservation. This is admitted by most to have an injurious effect, inasmuch as it is liable to produce too great a struggle for existence and a consequent weakening of the birds on the one hand, and, on the other, a possibility, owing to the great number of birds left, of cases of disease lingering on through the winter as mild cases, but ready in the following spring to cause an epidemic of the severe fatal disease (see below). Dr. R. Farquharson, in a letter to the Lancet, Sept. I874, was the first to state the opinion that the grouse disease was of the nature of a contagious fever, since "the idea of an epidemic and infectious fever fits the facts already obtained better than anything else" (Grouse Disease, Macdonald, p. I29).

He argued that, as great differences prevail in the degree of loss of flesh observed-some dead or dying birds being found as plump as in their healthy condition, whilst others are found reduced to mere skeletons,- this is in favour of its specific or constitutional nature..." so that whilst those dying from a very acute attack, or whose constitutions are unable to withstand its debilitating influence for more than a brief period, retain the outward appearance of health, 
others present all the conditions of excessive exhaustion " (l.c. pp. I 30 and I 3 I).

I may here at once state that this explanation offered by Dr. Farquharson, as to the difference of condition observed in birds dead of the grouse disease, is one which I think perfectly correct and one for which I shall be able to offer direct experimental evidence. Moreover, it is one which from analogy, i.e. such as is observed in other infectious fevers, would, a priori, suggest itself. The next noteworthy contribution made to our subject is by Mr. Andrew Wilson, Lecturer on Zoology and Comparative Anatomy, Edinburgh, who in the Edinburgh Medical Joumal gave the first accurate description of the pathology of the disease. "Mr. Wilson has had many opportunities (Macdonald, pp. I 45 and I46) afforded him for the examination of grouse dying from the disease, and he states that, in most of the birds he examined, he observed a markedly congested appearance of the mucous surface of the digestive and respiratory tracts. Repeated dissections and careful observations satisfied him beyond doubt of the almost invariable presence of this lesion in birds." Mr. Wilson "unhesitatingly" prefers the theory of Dr. Farquharson, i.e. that the grouse disease is an infectious fever; "and the reasons for enlistment under the banner of the epidemic hypothesis, rather 
than under that of parasitism, are chiefly those derived from the consideration that the former theory fully comprehends all the conditions which the phenomena of the grouse disease may present; and further, that the theory may be applied to the explanation of points and facts, of which the theory of helminthiasis can take no heed.

"We thus find that these birds are almost invariably affected by both kinds of entozoa-most specimens to a moderate extent, some to a very great degree. But it is exceedingly rare to meet with a bird in which not a single parasite of either kind is present. Out of many cases examined not a single bird was found actually free from the characteristic tape-worm, and in one or two doubtful instances only could it be asserted or suggested that none of the round worms were present.

"That the presence of these parasites was unconnected with the death of the great majority of the birds examined is, I think, proved by the want of any causal or obvious relationship between the infestation and the fatal issue; and also by the absence, in most cases, of the signs of fatal parasitism-such as inanition, producing the 'pining' condition, actual perforation, morbid appearance of the muscular tissues, etc. No one can deny that the presence of parasites in large numbers undoubtedly causes death in a 
specific and readily understood manner; but this fact will not explain the nature of the cause or disease which operates so fatally, without any exceptional development of parasites, and certainly without the slightest appearance of inanition or other concomitant symptoms of helminthiasis. Outside the parasitic hypothesis, applicable as that theory is to a certain class of cases, there lies, I am convinced, the great bulk of fatal instances, the exact cause of which fatality must be sought for in some lesion analogous to that involved in the idea of the epidemic theory. We must thus account for the death in numbers of grouse which, with what one may consider a normal and actual degree of parasitism, yet die and succumb to disease, and which on examination present wellnourished bodies, firm, healthy, muscular tissue, and other signs of presumed health" (Macdonald, pp. I47 and I48). 


\section{CHAPTER II}

\section{SYMPTOMS AND PATHOLOGY}

IT must be obvious that in a disease affecting birds that cannot be kept in captivity for any sufficient time, the accurate and systematic observation of the symptoms of the disease is fraught with great difficulties, and it is therefore impossible to give a connected account of the course of the disease. From what I can gather of shrewd and observant keepers, and what I myself observed on the grouse in I887 while staying on the moors in Ayrshire and in Cumberland, thanks to the editor of the Field, and the kind and generous hospitality of Mr. G. Bailey Worthington, then holding Blairquhan, and of the late Sir Frederick Graham of Netherby Hall, on the borders of Cumberland, and also from what I observed of ammers and other birds experimentally infected with the disease in the laboratory, I fully agree with Dr. Farquharson that the grouse disease is an acute infectious fever-being, in fact, an acute 
infectious pneumonia. In the severe forms, such as are observed during epidemics in May and June, the birds, once affected, rapidly, in a day or two, show great loss of muscular power and seek the water. The loss of muscular power is apparent by the impeded flight, this being sluggish, being performed with unwillingness, and not for any normal time, that is to say, if on the wing the birds soon drop again. Wherever there is a trace of water the affected birds will seek this, and in many instances in severe epidemics their bodies are picked up in numbers on the shores of such water. As the postmortem examination shows, the congestion of the lungs forms a conspicuous pathological symptom; and it is therefore clear that, as in acute congestion of the lung in man and animals, also in the grouse, under these conditions, fever must be a prominent symptom, and the above appearances during life easily account for this.

Also the peculiar alteration in the call of the diseased birds, this being somewhat feeble and hoarse, would be a natural result of the lung congestion, since this condition would prevent the birds from uttering their call with normal force, and the congested state of the mucous membrane of the larynx would cause the sound to be less clear and sharp, and more hoarse. The more or less ruffled 
condition of the feathers of the back and throat, or rather the absence of the smooth condition and metallic lustre of the feathers of the back observed in diseased birds, could also be accounted for by their feverish condition.

The colour of the eyelids, bright in the normal state, is not of the normal bright orange colour in birds dead of the disease. In every severe congestion of the lungs, such as obtains in the severer forms of the grouse disease, there is a general venous congestion of all parts consequent on the engorgement of the venous part of the heart supplying the pulmonary artery; and therefore also the skin all over the body, including the eyelids, would participate in this venous congestion. The natural aspect of the bright pigment of the eyelids would therefore become altered by the presence of this venous congestion, and the eyelid would look duller.

Pathological appearances.-The birds that die during a severe epidemic, such as obtains in the spring and early summer, show the following post-mortem appearances: the crop is generally more or less full of undigested heather; as a rule this is the more conspicuous the better nourished and the plumper the bird, and the better preserved the pectoral muscles are. I have dissected birds which were emaciated, the legs bare of feathers, and in which 
the crop contained only little or no food; and in such cases the disease of the lungs is as a rule, but not without exception, not very extensive, only a portion of one or both lungs being congested. Such is the case in some birds in the spring and summer epidemic; such is the case in birds that die in the autumn. In the majority of instances in which the dead birds are found plump - as for instance during the height of the spring epidemic-the lungs show a great deal of congestion, the crop is full, and the legs are not bare. I conclude from this that, in these cases the disease being severe, it rapidly kills the animal, or more correctly, if the animal rapidly succumbs to the disease it will show less loss of flesh, and the crop more full of heather than when the animal lingers on for some time-for then the fever would inevitably cause great loss of flesh, and the animal, as is to be expected, would present appearances of emaciation. This view, enunciated by Dr. Farquharson and Mr. Wilson, seems to me the best to harmonise with the facts.

The chief changes are undoubtedly those found in the lungs. The mucous membrane of the larynx and trachea is of a dark colour, hyperæmic; this is the more pronounced the more marked the lung change. The latter shows deep coloration in the greater part of either one or both organs, 
the hind portions being, in these cases, the ones chiefly affected; or both lungs are uniformly congested, being in some cases of a dark purple-red colour. On microscopic examination it is found that in the congested parts the large and small bloodvessels are uniformly distended and filled with blood, and that the air-spaces of the more deeply affected parts are uniformly filled either with a homogeneous or granular exudation, or with blood; so that in these parts we have a solidification of the lung which compares with the condition known as the red hepatisation in Pneumonia. There is, however, no fibrine in the form of threads noticeable in the air-spaces; the smaller air-spaces contain blood en masse, while the large ones are filled with a homogeneous albuminous exudation. From this we conclude that rupture of small vessels had taken place during life (Fig. I).

The spleen is not enlarged, and appears of a dark colour. The liver is uniformly congested and soft; it is either of dark red colour or appears almost black. On microscopic examination the large blood-vessels as well as the capillaries of the lobules are distended and filled with blood corpuscles. In some cases the liver, on post-mortem examination, is blackish, or rather is of a dark olive-green colour. In these instances the liver cells appear granular and more or 
less disintegrating, and contain dark brown pigment granules; besides, numerous clumps of dark greenish pigment masses are found in the spaces of the intralobular capillary vessels, while the large vascular branches are full of blood. Evidently in these cases the disease is of longer standing than a few days, for the capillary blood-vessels are not found filled with blood corpuscles, as would be the case in recent cases, but contain the remnants of disintegrated blood corpuscles, viz. masses of amorphous pigment. The dark greenish-black condition of the liver is always associated with great emaciation of the animals, and, as is proved by microscopic examination, is associated with a large amount of blood pigment, so that from this we conclude that there must have existed, some time before death, stasis in the blood-vessels, disintegration of the blood corpuscles, and change of the blood pigment. This is in agreement with what we mentioned on a previous page as to the emaciation of birds indicating that the disease must have lasted for some time. In all instances, however, the liver cells themselves contain brown pigment granules. The kidneys are congested, in some instances leading to hæmorrhage into the tissue of the kidney. The intestinal mucous membrane shows patchy congestion, and the same is the case with its serous covering, which in most instances is congested in many places, 
sometimes to a considerable extent. In some cases the peritoneum appears very moist in these localities; that is to say, there is a small amount of exudation. I have seen a few cases in which hæmorrhage has taken place, showing itself in the form of petechia in the peritoneum.

From this we see that the grouse dead of the disease show a definite series of pathological appearances, in which the congestion of the lungs and liver is very conspicuous.

The above considerations led me to conclude that the disease was an acute infectious disease, and owing to the constantly congested and diseased condition of the lungs and liver, I thought it possible that the disease belonged to the group of diseases in which the microbe caused the disorder by its multiplication in the blood. The first birds dead of the disease which I dissected in June $\mathbf{I} 887$, in Ayrshire, were examined with the view of discovering the microbe in the blood. Cover-glass specimens were made of the heart's blood and of the blood of the lung, i.e. a thin film of blood was dried on cover-glasses and then stained in the usual manner with aniline dyes -methyl blue or gentian violet; but on microscopic examination no bacteria could be discovered. Numerous tubes, some containing nutrient gelatine, others nutrient Agar, were inoculated in the usual 
manner with the heart's blood; they all remained sterile on incubation and free of any growth. The heart's blood of at least half-a-dozen grouse dead of the disease, or killed while diseased, was thus examined, but no bacteria could be discovered in it. Lungs, liver, and kidneys were taken out from the animals after death, and were cut into small bits and preserved in spirit or Müller's fluid. Sections were then cut and stained with aniline dyes and mounted in the usual way, but here also no bacteria could then be found. This was the position of things which I described in my first report in the Field in July I887. I have in I888, I889, and I890 examined many birds that died of the disease in April, May, June, or July; and also in them I could not discover in the blood of the general circulation, either in cover-glass specimens or by cultivation, any bacteria. From this I am enabled to say that in a large number of the grouse dead of the grouse disease in the spring and summer,- - that is, dead with the characteristic pathological appearances of the lungs and liver,-the blood of the general circulation does not contain any microbes discoverable by the cover-glass or culture test. In I 888 therefore I directed my attention to examining in the same way, in the fresh condition, the tissue of the lung and liver; and, with the exception of one or two doubtful cases, in 
all others, whose number is considerable, I obtained, by the culture test in nutrient gelatine, evidence of the presence in the lungs and the liver of numbers of bacteria belonging to one and the same species; for when a particle of the lung or liver tissue is rubbed over the slanting surface of nutrient gelatine, or when it is shaken up in a test-tube with melted sterile nutrient gelatine, and the latter is then poured into a sterile glass dish, allowed to set and then incubated-that is if a plate cultivation in gelatine is made-colonies of one single bacterial species are obtained. By making sections through the inflamed lung or liver, after hardening, and staining these sections for several hours in methyl blue, or better still in rubin and methyl blue, it is found that in some parts some of the capillary blood-vessels, both in the lung and in the liver, contain continuous masses, plugs or emboli, of the same bacterial species (Figs. 2, 3, 4, I 5 and I6). ${ }^{1}$ But there are seen under the microscope extensive parts in which the blood-vessels do not contain them. It is from this easy to see why I failed in I 887 to obtain the microbe from the lung or liver in coverglass specimens or in culture, viz. I used in both cases a droplet of the blood of the lung or liver,

1 In sections through the hardened liver stained with rubin, then with blue, the clumps of microbes are brought out with great distinctness as blue masses on a red ground. 
and from what has been stated just now it is clear that it would amount to an extremely distant chance to get some of those capillary plugs in the droplet of blood. In ISSS I made a good incision with sterile scissors or scalpel into the substance of the lung or the liver-the lung or liver as a whole having previously been well dipped in saturated solution of mercuric bi-chloride to disinfect the surface - and from the cut surface a particle of the tissue was cut out with sterile scissors and used for cultivation; here, of course, the chances of hitting one of the capillaries containing the bacterial masses are very much greater. And working on this plan I have obtained sufficient evidence to enable me to state confidently that the lung and liver of the grouse that are affected with the disease in the spring and early summer contain a definite species of bacteria forming continuous masses or plugs in some of the capillary blood-vessels, but that in the blood of the general circulation they cannot be demonstrated. From this then it follows that the bacteria find their way into the lung and liver, in whose capillaries they settle and by their multiplication produce those embolic plugs. This is nothing exceptional, since we know of infectious diseases, both acute and chronic, in which the specific microbe does not thrive in the circulating blood, i.e. does not find in this a suitable nidus, and is there- 
fore absent from this, though it uses the blood circulation for getting to its proper nidus-in our case the lung and liver. In human croupous pneumonia it is the lung; in tuberculosis the lymphatic tissues, lung, liver, and spleen. 


\section{CHAPTER III}

CIIARACTER OF THE BACILLUS OF THE GROUSE DISEASE

In the grouse disease the cultivations obtained, as I said above, in almost all cases are of exactly the same character, and it is these which I now proceed to describe.

\section{(I.) Cultural characters :-}

The characters of the microbe in the gelatine plate, in gelatine stab- and streak-cultivation are quite distinct. After $24-36$ hours' incubation at $20^{\circ} \mathrm{C}$. of a gelatine plate, the first indications of the superficial colonies are noticed as grey, translucent, flat, angular dots, visible under a magnifying-glass. They spread rapidly in breadth, so that after 3-4 days they reach a diameter of 3,5 , and more millimetres; they remain flat and thin and are slightly thicker and folded at the margin, which is much crenated. In reflected light their aspect is whitish; they look dry, and when viewed obliquely have a fatty satiny lustre. In transmitted light they look pale brown and trans- 
lucent. About the end of the week their maximum growth is reached (Fig. 5). As with other species, so also here, when the colonies of the surface are placed at great intervals they grow much larger than when they are near together. Those that develop in the depth of the gelatine plate are very small as compared with those growing on the surface, and remain minute round dots, whitish in reflected, brownish in transmitted light. The gelatine is never liquefied by the growth.

Sub-cultures made of a colony on the surface of gelatine as streak-cultures,-i.e. when with the end of a platinum wire a colony is touched and is then drawn in a line over the surface of the gelatine,--show after one or two days along the line of inoculation a narrow, grey, translucent band. This band rapidly broadens, and remains flat, and possesses a crenated margin. After a week its breadth is 5-ro millimetres and more, it is flat, thin, translucent, dry, smooth, and possessed of a satiny or fatty lustre; the margin is slightly thicker than the middle, translucent, and plicated. In stab-culture the line of inoculationthe stab-becomes visible after a day or two as a grey line. Under a glass it is composed of minute spherical dots. On the upper surface of the stab a thin, translucent, whitish-grey film graçually spreads over the gelatine with irregular margin, which in 
about a week has covered nearly the whole free surface of the gelatine in the test-tube. But even at the maximum growth the clots constituting the stab remain small (Figs. 6, 7), and are brownish in transmitted light.

After a few days' incubation of the stab culture there are generally in the deeper parts one or two large flat gas-bubbles to be noticed, fixed on to the growth with one end, and placed, therefore, eccentrically to the line of the growth. The gas-bubbles slant generally in an upward direction. The development of gas-bubbles in the deeper parts of the growth is well and easily shown by making a "shake culture" in gelatine-that is to say, by distributing (by shaking) a limited number of the microbes in melted nutrient gelatine contained in a test-tube and then allowing this gelatine to set, by keeping it in cold water for a short time before incubation. After two days numerous colonies are visible as minute dots distributed through all the layers of the gelatine, and attached to each dot or colony in the deeper parts is a small flat gas-bubble. Such a culture looks extremely characteristic (Fig. 8). The gasbubbles increase in size, and, if the colonies are numerous, i.e. if a considerable number of the microbes had been originally introduced into the gelatine, the lower half or two-thirds of the culture 
are crowded with gas-bubbles, the upper layers of the gelatine remaining free from them. Gradually, however, the gas-bubbles disappear, i.e. escape on to the surface, and by the end of eight or ten days all but the deeper situated bubbles have clisappeared. The escape of the gas-bubbles between the gelatine and the glass wall of the test-tube can be easily ascertained.

On nutrient Agar at $36-37^{\circ}$ C. the microbes grow with great rapidity; in two or three days the surface of the Agar becomes covered with a greyishwhite thin film.

In alkaline beef broth (with I per cent peptone), incubated at $36-37^{\circ} \mathrm{C}$, there is, after 24 hours' growth, strong uniform turbidity, which reaches its maximum in about 3 days; at the same time there is a copious greyish floccular or granular precipitate. After about 5-7 days there is noticed, just at the point where the surface of the fluid is in contact with the glass of the test-tube, a ring of whitish growth; but there is at no time a distinct pellicle formed on the surface. On potato, previously sterilised in the steam steriliser, the microbe grows well at $36-37^{\circ} \mathrm{C}$., forming in a few days a light yellowbrown, limited film, which looks like paint, and does not spread much over the surface of the potato. 
(2.) Character of the microbe in the fresh state:-

The microscopic examination of the microbe in the living or fresh condition, i.e. in a drop of saline solution, or serum, or broth, shows it to be a spherical, or more generally an oval corpuscle, occasionally more distinctly rod-shaped or cylindrical, with rounded ends. The microbes occur either single or, more commonly, double, i.e. as dumb-bells (Fig. 9). The great majority are without any movement of their own, showing only the oscillating movement that all granules suspended in a fluid show, namely, the Brownian molecular movement. But occasionally one or the other shows active locomotion, either spinning round and round, or darting, under oscillation of its body, through the field of the microscope. Such actively motile individuals are to be seen fairly frequently in a fresh preparation made of a recent colony from a gelatine plate, or from a recent gelatine tube-culture, or from Agar culture, from the fresh lung-tissue or from the liver. By means of the platinum wire a particle of the culture or of the tissue is suspended or distributed in sterile salt-solution-or better, in broth—and examined either in the "suspended drop," i.e. in the hollow glass slide, or simply mounted on an ordinary glass slide. The number of non-motile bacteria is always, under the most favourable conditions, greatly in 
excess of those that are possessed of motility; the number of these latter being limited. In preparations made of recent culture, except potato or broth cultures, the number of motile forms is appreciably greater than from a culture of some days' standing; in older cultures they are missed. If of any gelatine cultivation, say a streak culture or a plate culture, fresh preparations are examined from day to day, the same tube or the same colony being used, it is noticed that while in the first few days the number of motile bacteria is sufficiently great to see in every field several or even many examples, about the end of the week, and later, few motile forms are to be found in the whole slide. And if now fresh sub-cultures are started, it is again noticed that, while recent, they show the motile forms fairly abundant; if of some standing, only non-motile ones are present. I conclude from this that there is produced in the cultures, by the growth, a chemical substance which inhibits and is adverse to the motility. It cannot, I think, be explained by saying that the later offsprings are devoid of the organs of motility, i.e. the flagella, while the earlier offsprings possess them, because when they are planted on fresh nutritive medium they again show motility, and on potato or broth, even after 24 hours' growth no motile forms are found. It can hardly be said 
that in one case they produce offsprings with flagella and in the other without. I have not examined them with regard to demonstrating the presence or absence of flagella, and therefore cannot say positively that it is not so, but it seems to me more probable that the loss of motility is due to some chemical poison produced by the bacteria. In the fresh blood of grouse (dead in the late summer and autumn) the bacteria are always present, as I shall point out below, and motile forms, though not very abundant, can nevertheless be easily detected. So also in the fresh blood of animals and birds infected by inoculation with the cultures of the microbe (mice, guineapigs, yellow-ammers, and buntings), motile forms can be found. In the grouse and in the sub-cultures from the grouse the oval forms abound. Some are so short that they appear like cocci; but it must be added that, though at first sight many appear like spherical cocci, on more careful focussing with high powers it can be shown that in reality most of them are oval, but appear spherical when viewed foreshortened or in optical transverse section. In sections through the liver or lung of the grouse dead of the disease, or of other animals infected with the cultures, the blood capillaries, as mentioned above, are found plugged with the microbes. Now it is precisely in such masses that the individuals, 
even under a moderately high power, e.g. under a power of 300 to 400 , appear as spherical cocci; but on examining carefully with oil immersions the marginal parts of the masses, where the individuals are loosely arranged, it is clear that most of them are distinctly oval or even rod-shaped or cylindrical.

When the microbe is examined in mice or guineapigs that have died after inoculation with the culture, it is found that the number of rods and cylindrical forms is very much greater than in the grouse or in the sub-cultures of the grouse. This is well shown in Figs. IO and II.

In the ammer, bunting, greenback, and finch, which are highly susceptible to the disease, many individuals are rod-like or cylindrical; and also, in the blood of the grouse dead during late summer and autumn, rod-shaped individuals abound.

Whatever the source of the organism when grown in broth,-or better still, when grown in or on nutritive gelatine to which 5 per cent. solid sea salt (Stedman's sea salt) has been added,- the bacteria grow out into long cylindrical threads, most of them smooth and uniform, but some made up of individual cylindrical bacilli (see Fig. I2). There can then be no doubt that we have to deal with a form which corresponds to those groups of bacterial species known as bacilli. 
Measurements made of the organisms in coverglass preparations, dried and stained, give the following figures :-

The spherical forms are $0.4 \mu^{1}$ in diameter ;

The oval forms are $0.6 \mu$ long, $0.4 \mu$ thick ;

The rods and cylindrical forms o.8-1.6 $\mu$ long, $0.4 \mu$ thick.

II $\mu=0.00$ I millimetre, or about $\frac{1}{25000}$ part of an inch. 


\section{CHAPTER IV}

\section{EXPERIMENTS WITH CULTURE BY SUBCUTANEOUS INOCULATION}

Experiments were made on tame mice with cultivations,-either with a particle of a gelatine- or potatoor Agar culture distributed in, or mixed with, sterile salt solution, the mixture being slightly turbid; or with a broth culture $24-48$ hours to 3 days old. Of the salt mixture or of the broth culture a few drops (3-5 minims) are injected under the skin of the back. The following day the animals are found quiet, and they do not feed well; the eyes are almost closed, the back is curved, the coat rough, they look "lumpy"; the breathing is rapid, and, while motility becomes weaker and weaker, they die either before 36 hours are over or during the following night, i.e. before 48 hours. If the inoculation is made with a broth culture 24-48 hours old, most of the mice inoculated are dead before the end of the second day; some live till the end of the third day; a few remain quiet and ill and gradually recover. In the latter cases a 
more or less extensive necrosis of the skin and subcutaneous tissue at and about the seat of inoculation is noticed.

As stated just now, the majority of the mice inoculated with a recent broth culture (3-5 minims) die. Gelatine cultures less than ro days old, and potato- or Agar cultures less than 4 or 5 days old, used as a slightly milky salt mixture, prove virulent: old cultures of gelatine and old cultures of Agar are apt to show less virulence, this may become almost lost with age. In the former case a similar proportion of the mice succumb, but later than after inoculation with recent cultures; in the latter no death follows on inoculation of even large doses. In order to ensure virulence I use recent broth cultures. These do not fail, no matter whether they are sub-cultures of old or recent cultures on gelatine, Agar, or broth. I have tested in this respect cultures that have been carried on in sub-cultures for many generations-in gelatine for more than three years and a half; and I find that when a broth sub-culture is made of an old gelatine culture, say one or several months to half a year old, and is inoculated into mice, it proves virulent. The highest degree of virulence is obtained by making a sub-culture in alkaline broth to which a piece of boiled white of egg is added (the whole being sterilised before use). 
The vitality of the microbe is a very great one, since gelatine cultures, kept at the temperature of the room for more than a year and a half, proved still alive, i.e. good and virulent sub-cultures could be easily obtained from them.

On making a post-mortem examination of the mice that die, the following appearances are constantly found:-Right heart contains fluid blood, left heart contracted. The lungs show œdema and congestion; this latter extends generally over both organs, and is marked by their being redder than normal-sometimes deep red or dark purplered. The liver is dark red, and congested; the spleen is of a dark colour, and slightly enlarged; both kidneys are congested. At the seat of inoculation the subcutaneous tissue shows sometimes slight, sometimes distinct, congestion, or even sanguineous infiltration. In the heart's blood the microbes can be easily detected by cover-glass specimens, and particularly by cultivation; a droplet of blood yielding, in plate cultivations, or in tube cultivations (rubbed over the slanting surface of solid nutrient gelatine by means of a platinum wire), sometimes many, sometimes innumerable colonies. A section having been made with sterile scissors into the tissue of the congested lung, a cover-glass impression is taken of the cut surface; this is then dried 
and stained in methyl blue or gentian violet, washed in water, dried, and mounted. Examined under the microscope, it shows the outlines of the air-cells marked by innumerable microbes: round or oval forms, singly and particularly in dumb-bells, rods singly and in dumb-bells, and a few cylindrical forms (Figs. I 3 and I4). In some cases their number is not so great, but in acute fatal cases they are always easily discovered in cover-glass specimens made of the lung juice. Cultivations made of the lung tissue, the liver, and the spleen, always yield a great abundance of colonies. As the result of inoculations made with the culture of the grouse into the subcutaneous tissues of guinea-pigs, about 50 per cent of the animals die during the third or fourth day; the others, though they survive, develop a firm tumour at the seat of inoculation. But all the guinea-pigs are distinctly ill 24 hours after inoculation; they show a slight soft swelling at the point of inoculation, and are quiet and off their food; some grow weaker during the second and third day, cannot move, are shaky when they attempt to move, and gradually sink and die. At the post-mortem examination the subcutaneous tissue at and about the seat of inoculation, on the abdomen, and even on the chest, is œdematous and shows hæmorrhage. The blood of the right heart is fluid, both lungs are much congested; 
the liver is also congested; the spleen is not enlarged, but dark; the peritoneum, the kidneys, and the suprarenals are much congested. The peritoneum is moist, and there is sometimes a small amount of exudation; petechix are often noticed in the omentum. The heart's blood, and particularly the lungs, contain the microbes in large numbers (Fig. I7). Cover-glass specimens and cultivations of the blood, and particularly of the lung juice, show the microbes in very large numbers. So also the peritoneum, especially the serous covering of the liver. In the heart's blood the microbes occur as single or dumb-bell oval bodies, and as rods; they are either in the plasma or they are contained within the white blood-cells-in the latter case the cells are very much swollen up, and some are disintegrating. In Fig. I 7 this condition is well shown.

[The intracellular occurrence of the bacilli in the guinea-pig, and, as will be mentioned presently, also in the ammer, does not indicate that there is going on a process of combat on the part of the cells as against bacilli, as is only too readily assumed by those who with Metschnikoff assume this to be the case wherever cells are met with that enclose bacilli; for in our cases we are dealing with a disease which is rapidly fatal, and in which the bacilli abound in the blood.] 
As regards the shape and motility of the microbe in the guinea-pig, the same holds good that has been said of the mouse.

The inoculated guinea-pigs which survive show at the seat of inoculation a firm flat tumour which extends on to the chest. About the end of ten days or so, necrosis and sloughing of the indurated part of the groin sets in, which gradually leads to complete healing.

In several instances guinea-pigs which had been inoculated with virulent culture, but had survived, were, at the end of twelve to fourteen days, re-inoculated with a virulent culture, but no result followed this second inoculation.

Rabbits inoculated subcutaneously with culture that proved virulent on mice and on guinea-pigs show only a local result. A thickening about the seat of inoculation, due to caseous purulent infiltration, sets in, but remains circumscribed, and in some cases opens as an abscess, which rapidly heals; or the tumour becomes firmer and smaller and gradually disappears. Only once have I seen a rabbit die during the second day, but neither cover-glass specimens nor cultivations revealed any microbes either in the blood or in the lung.

In pigeons and fowls the subcutaneous inoculation is not followed by any, not even a local, positive result, the animals remaining lively and well. 
The most striking results were obtained on the common bunting and yellow-ammer (greenback, cockbit, and finch), for the injection of a small drop of a broth culture into the thigh is followed by fatal results. The injected leg is swollen before the end of twelve hours, the birds do not move that leg, and sit perched on the other leg. Towards the end of the first day they are quiet, "lumpy," their feathers are ruffled, their breathing is very rapid, their eyes are closed; the animals are drowsy and have diarrhœa. Most of them die towards the end of the first day, about the 20th-22nd hour; some die at about the 24th hour;

a few survive longer-even to the fourth day. On post-mortem examination hæmorrhagic infiltration is found in the muscles of the thigh at the seat of the inoculation, the lungs are deeply congested, the liver is congested, and so is the mucous membrane and the serous covering of the intestine. The spleen is dark and small. There is then a complete analogy in these respects with the appearances found in the grouse. The blood of the heart and the sanguineous juice of the congested lungs teem with the microbes (Figs. 18 and 19), either as single or dumbbell spherical and oval forms, or as single or dumb-bell rods with rounded ends. Many of the white blood cells in the lung juice contain in their substance the bacilli: in some the bacilli are few in numbers, in 
others the cell substance is crowded with them (Fig. 20). Examined in the fresh state, some of the bacilli show mobility. In dried and stained cover-glass specimens and in cultivations from the heart's blood or lung juice; in plates, gelatine streak and stab, in Agar, on potato, and in broth, the microbe shows the same characters as that of the mouse (Fig. 2I).

The buntings and ammers possess even greater susceptibility to the disease than the mice; this is shown by the fact that gelatine and Agar cultures, which through age have become attenuated for mice and guinea-pigs, still produce fatal results in these birds.

Sections made through the lung show many capillaries and small vascular branches almost completely blocked by continuous masses of the microbe. Staining the sections in rubin ( 2 per cent) for two or three hours, then washing in water, then staining in methyl blue aniline water for seven or eight minutes, washing, dehydrating, passing through xylol, and mounting in Canada balsam solution, shows the cell nuclei and the bacilli blue, the rest of the tissue red.

Sparrows were also inoculated subcutaneously. The great majority (7o per cent) die under the same symptoms and with the same post-mortem appearances as the ammers; the microbes are present in the lungs 
and the heart's blood; in the latter, however, they are not numerous; in some cases they could not be found in the blood, though they were present in the inflamed lungs.

I have further tested, as regards virulence, the sub-cultures derived from the blood and lung of the mouse, and from the blood and lung of the ammer, on mice, guinea-pigs, and ammers, and have found that there is no difference between them and the subcultures obtained from the lung of the grouse. While, then, by these experiments it follows that the cultures of the microbe act virulently when subcutaneously injected into mice, guinea-pigs, buntings, ammers, and sparrows, they do not produce fatal infection in rabbits, and do not produce any result in pigeons or fowls; and for these reasons alone the microbe as well as the disease it produces is well defined from chicken cholera, which, as we shall show later on, is very characteristic as regards the virulence of its specific microbe on rabbits, fowls, and pigeons. As we shall also show, there exists a certain similarity in shape, in certain cultural characters, and in the non-liquefaction of the nutrient gelatine, between the microbe of grouse disease that we have been hitherto considering, and the microbe of chicken cholera; but the differences in the growth of the two microbes in the various culture media are sufficiently striking 
to enable us to say that they are two well-defined species.

Experiments of feeding and air infection :-

Numerous experiments were made on mice, guineapigs, and ammers to see whether, by introducing the bacilli into the digestive organs, infection could be produced. For this purpose broth cultures, the virulence of which was proved by subcutaneous inoculation, were mixed with the food given to mice and guinea-pigs, but in no single instance was any result produced. In the case of the ammers, several drops of the broth culture were directly dropped into the mouth, the beak being held open, and readily swallowed. No result followed.

From this it follows, then, that by ingestion the microbe does not act, and therefore must be said to be destroyed by the gastric fluid, and does not reach in a living state the small intestine.

It is otherwise with the respiratory organs, for infection by these has been ascertained in the case of the ammers. Several experiments were made in this respect; in one set a bird was inoculated with virulent culture, and then placed in a cage with two normal birds. The inoculated ammer was found very ill the next day, with the one leg drawn up, feathers ruffled, breathing very rapid, eyes closed. The bird died during the second day; the other two 
were found ill the day following the death of bird No. I, and died in the course of the third day with the symptoms and appearances of the disease; in their blood and lung the microbe was present in great numbers. In a second set two wire cages were placed close side by side; one contained an ammer that was affected with the disease after inoculation; this bird had been inoculated with attenuated culture (see below), and remained in the diseased condition for several days; in the adjoining cage were six normal ammers. The two cages were covered with one cloth. All the six neighbours became ill with the disease after two days, and died with the typical symptoms and appearances during the following day. 


\section{CHAPTER V}

THE AUTUMNAL DISEASE

IN I888, I889, and I890, I received, thanks to the editor of the Field, during August, September, October, November, and even December, many grouse from various parts of Scotland and the north of England which had died of the grouse disease, that is to say in which, on post-mortem examination, the congestion of one or both lungs, the congestion of the liver, the small dark spleen, and the patchy redness of the intestine and the peritoneum, were present. In many of these birds the heart's blood was examined for the presence of the specific microbe, both in coverglass preparations and by culture, and, unlike the birds dead during the spring and early summer in which the microbes are absent from the blood of the general circulation, the heart's blood of those birds always contained them in large numbers. In fresh specimens of the heart's blood they can be identified as oval or rod-shaped single or dumb-bell bacteria, some actively motile. As a matter of fact it was 
this observation which led me to re-investigate the microbes obtained from the spring grouse, for of them I had in my earlier communications on the subject stated that they are non-motile. On reinvestigation, I ascertained that this is not so, that in every specimen made of the cultures there are some individuals which show mobility, but the number of which, as stated above, decreases in older cultures, and which are absent in recent potato and broth cultures.

The bacteria in the blood are, as a rule, more rod-shaped than those taken from the lung, though also in the blood some are spherical or slightly oval; the rods are also here commonly arranged as dumbbells (Fig. 22).

Cultivations made with a drop of the blood in gelatine plates, or by rubbing a droplet of blood over the slanting surface of solidified nutrient gelatine, show, after 24-48 hours, innumerable colonies; the slanting surface of the gelatine is so covered with them that, having become in many places confluent by proximity, they form an almost continuous film. As regards the character of the microbes in coverglass specimens, fresh or dried and stained, made of the cultures, these microbes are identical with those derived from the lung, both of the spring grouse and of the autumn birds, and there is nothing to specially add to what has been stated in a former 
chapter. The microbe from the heart's blood cultivated in gelatine plates, in streak and stab in gelatine, in Agar, in broth, and on potato, is in every essential the same as already described; so that there can be no doubt that the microbe of the lung and liver of the spring grouse, and of the heart's blood of the autumn grouse, is the same in morphological and cultural respects; and since the pathological appearances of the grouse dead in the autumn are identical with those dead during the spring and early summer, we are justified in saying that the autumnal disease is also the real grouse disease. Now, on inquiry, it is found that the disease in the late summer and autumn is of less frequent occurrence; this is, of course, to be expected, seeing that the total number of the grouse rapidly diminishes during the shooting season. But when saying that the disease is less frequent during the summer and autumn, I am not referring to such a cause of diminution. I am referring to moors in which no epidemic disease has been prevalent during the spring, or in which no great amount of shooting has been going on in August. I have received birds during August, September, October, and the beginning of November, from moors in which very little or no disease had been present in the spring and early summer, and yet birds died now and then during the autumn. We may from this conclude that 
in these cases the disease, though present, was of the sporadic character. If this be the correct view, it would point to the danger existing on such moors, as far as they themselves as well as their neighbours are concerned, and it would explain at the same time how, by lingering on during the winter, an epidemic outburst of the disease may occur in the following spring. Though I do not think that this is the only way in which the spring epidemic may arise, it is at any rate one very probable way. It has been pointed out already, and will be again referred to, that the vitality of the microbe, as proved by experiments with cultures, is of a very high degree, and it is quite possible that the microbes left in a moor from a previous epidemic, and remaining sheltered in the soil under the heather, grass, or otherwise, may on a future occasion give rise to infection, and thus start a new epidemic. But the theory that cases of a previous spring and summer epidemic linger on through the autumn and winter as sporadic cases in any one part of a district, would furnish a more direct cause for an epidemic in the ensuing spring than the other condition, which supposes that the microbes derived from birds that died in the spring or summer may retain their vitality, and remain alive on the moors through the autumn and winter till the next spring, when they might give rise to fresh outbreaks. 
In this disease, as in others, certain accessory or secondary conditions are required in order to enable the disease to assume the epidemic character. This has been already mentioned; and now we wish to add that the microbe of the autumn disease seems distinctly less virulent than that of the spring disease. Inoculations of mice were made with recent broth cultures derived from the heart's blood microbe of autumnal cases, but in the majority of cases no fatal result was produced, although all the animals became ill. In a series of such experiments comprising ten mice, all were quiet and off their food the day after inoculation; one died after three, one after seven days, the other eight survived; while of six mice inoculated for control with recent broth culture of the microbe of the lung of the spring disease, four died in 48 hours, one during the third day, and only one survived.

Buntings and ammers so highly susceptible to the spring microbe are also found less affected by the autumn microbe; most of them die, it is true, but conspicuously at a later period than after inoculation with the spring microbe; they are ill for several days, some die after the fourth, others after the fifth day, their heart's blood containing the microbe in large numbers. To prove that the autumn microbe is of an attenuated virulence, I re-inoculated, after 
two to three weeks, those mice (80 per cent) which had survived the first inoculation with the autumnal microbe, with virulent culture of the spring microbe - tested on control mice-and found that they proved refractory, neither disease nor death ensuing.

These experiments seem to me at any rate indicative of a means of discovering a protective vaccine for the grouse, i.e. a material which would only produce a transitory illness without fatal result. For if cultures of the autumnal microbe became still more attenuated, say by certain age of the sub-culture, it might be found just of the sufficient degree of activity to cause slight disease, transitory in character, but protective against infection with the fatal disease. To ascertain this a long series of experiments would be required, which I have not had leisure to make. I have myself made some experiments with the gelatine sub-cultures of the autumnal microbe; these sub-cultures were one week old, and were inoculated into mice, but though the animals showed slight illness, no fatal result followed; and I can quite imagine that if such or similar cultures were tried by inoculation on young grouse on the moors, - a not at all impossible proceeding considering that most keepers know of most of the grouse nests, -it might be possible to produce a slight transitory illness, protective of the fatal disease. But this is a 
suggestion, the carrying out of which, from what I know of moor-owners, would meet with great diffculties; difficulties not on the part of scientific men, who, I am sure, would be willing to undertake such work, but on the part of the grouse-owners themselves, who, with some notable exceptions, would probably be unwilling to consent to such experiments unless immediate success could be guaranteed. 


\section{CHAPTER VI}

FURTHER CHARACTERS OF THE BACILLUS

OnE of the important points in the life-history of a bacterium in general, and of pathogenic microbes in particular, is the question of spore formation. A bacterium that is possessed of the power of forming permanent seeds or spores possesses at the same time the power of preserving vitality and of insuring new generations to a far greater extent than one that is not possessed of this power, because spores are, as a rule, capable of retaining their vitality and the power of germinating under conditions and circumstances such as easily affect the vitality of non-spore-bearing forms, e.g. heat, cold, drying, absence of sufficient nutriment, chemically obnoxious substances. While it is well known that continued cold, even if above freezing-point, or heat far below the point of boiling water (even $55^{\circ} \mathrm{C}$.), acting for many hours, or deficiency of nutriment, drying, acids and alkalies, affect considerably the vitality of non-sporebearing forms - many in fact perishing under those 
conditions - those that form spores, and as spores, resist all the above influences. In the case of pathogenic bacteria those that form spores, e.g. the bacillus anthracis, the bacillus of the classical malignant œedema, the tetamus bacillus,--having formed spores, as spores are, for the above reasons, of the utmost importance in etiological respects; for the spores, even when finding themselves under very unfavourable conditions, retain their vitality, and, having access to a suitable animal body, are capable of setting up the disease. In the case of non-sporebearing forms this can only happen if they are fortunate enough to be shielded in one way or another from these obnoxious influences.

Now the bacillus of grouse disease belongs to the group of pathogenic organisms which do not form spores. On microscopical examination of fresh and of stained specimens there is no indication of the presence of anything that will compare with the spores in those species of bacilli in which spores do occur, and the experiments on the influence of heat and of drying fully bear this out. A culture of the grouse bacillus exposed to a temperature of $60^{\circ} \mathrm{C}$. for five minutes becomes hereby completely sterilised; no new sub-culture can be produced from it. Or, if a culture medium in a test-tube or flask is inoculated with the living grouse bacilli, then exposed to $60^{\circ} \mathrm{C}$. for 
five minutes, and then incubated, it is found to remain free of any growth - that is, to remain sterile; while a culture tube not exposed to this temperature, on incubation produces good and normal growth.

Whatever the age of a culture, and whatever the medium in which the bacilli have been growing, a temperature of $60^{\circ} \mathrm{C}$., acting for five minutes, kills all life of the bacilli of the grouse disease. This fact is, so far as our present knowledge goes, sufficient to justify us in concluding that in those cultures no spores have been formed.

I have made experiments with regard to the possibility of attenuating the virulence of the bacilli by temperatures below the killing point, viz. below $60^{\circ} \mathrm{C}$., in order to obtain easily procurable definite materials by which, on inoculation, owing to the decreased virulence, a slight transitory ailment could be produced which would protect against a second fatal infection; for I had already ascertained, as mentioned on a former page, that mice, after inoculation with attenuated virus (the cultures derived from the blood bacilli of the grouse dead from the autumn disease), and after recovery from the transitory illness, remain refractory to a second inoculation with virulent cultures.

I exposed, therefore, virulent broth cultures to a temperature of $50^{\circ}$ Fahr., five, ten, fifteen, and twenty 
minutes respectively, and then tested their virulence by inoculation on mice and buntings, but no difference in the virulence of the so-heated cultures could be discovered, for the animals inoculated with them died in the same way and time as the control animals inoculated with the culture previous to the heating. I then tried heating the virulent broth cultures to $55^{\circ} \mathrm{C}$. for ten minutes and for twenty minutes respectively. The result was unsatisfactory, since the cultures so heated proved still virulent. I need hardly add that cultures heated to $55^{\circ} \mathrm{C}$. for twenty minutes contain the bacilli in a living condition. Sub-cultures made from such heated cultures all yield normal good growths on inoculation.

In connection with the action of heat on broth cultures, I wish to mention here the following :-

Broth cultures incubated from two to three days at $35-37^{\circ} \mathrm{C}$. contain chemical substances which themselves are capable of producing the death of mice on inoculation in sufficient quantities, for when such a broth culture is sterilised, i.e. is exposed for five minutes to $60^{\circ} \mathrm{C}$., when hereby all the bacilli are killed, and when from such a sterilised broth culture five minims are injected into mice under the skin of the back, the animals die under the same symptoms and in the same time as the control mice inoculated with the same amounts of the same broth 
culture previous to sterilisation. Of course the mice that die after being inoculated with the sterilised broth culture do not contain any bacilli either in the heart's blood, or lung, or spleen. In a later chapter, after having described and discussed a species of pathogenic bacilli (ærobic bacillus of œdema) occurring in the soil, which is in many respects similar to the bacillus of grouse disease, I shall have occasion to return to an interesting physiological action of the grouse bacilli when inoculated simultaneously with the ærobic odema bacillus. At present I wish to conclude this chapter by the observations I made with regard to the influence of drying on the bacilli of grouse disease.

The experiments of drying a particle of the growth prove that hereby the vitality of the bacilli is completely annihilated. Of a living broth culture a thin film is spread over a sterile cover-glass (sterilised previously by heat), then kept - covered up, while the film is dried either over a dish of sulphuric acid or in the air-pump: if with such a dried film a tube containing nutrient broth is inoculated and incubated, the broth remains sterile, proving that the drying has killed the bacilli. But the experimental result is different-if, for instance, a particle of the growth is removed from a culture on gelatine, Agar, or potato, and dried on a cover-glass. In a percentage of cases 
it will be found that the particle, though apparently dry, nevertheless still includes living bacilli; for on inoculation of broth with it, and after incubation, the broth becomes turbid with the grouse bacilli, a new growth having taken place. This is evidently due to the fact that the particle becomes dry on the surface only; the central portion not becoming quite dry, being protected by the outer dry part, still harbours living bacilli. The larger the particle, the greater the chance of the bacilli surviving in the centre. Now this may be applied also to the conditions obtaining on a moor. A bird dies of the disease on the moors. Its body becomes lacerated or opened in some way, by animals of prey or otherwise, portions of the lungs and liver dry up, and may retain living bacilli which may remain sheltered for some time, and, when finding access to a suitable bird, say next spring, may set up the disease, and thus produce a focus for the development of the epidemic. I do not mean to say that such an occurrence can be or has been proved, but it must be admitted that such an occurrence is possible, and that it is justified by the experimental facts with regard to the influence of incomplete drying.

From all that has preceded it will be evident that the only measures of dealing with the disease in order to check its spread and epidemic appearance, 
lie in the direction of what is popularly known as the stamping-out process. Any grouse on the moors at all suspicious at any time should be destroyed, and every grouse thus destroyed or that dies of the disease should be carefully removed and burned. I am not sure that those who burn the heather on moors on which grouse-disease prevails are not following a right plan, for the reason that hereby they remove that which most likely harbours the adhering infective material; nor am I sure that, on moors on which disease has been present, an extensive battue of grouse would not also remove many of the birds that have the disease in a mild form, and that might carry it on, as it were, through the autumn and winter. It is therefore especially important that during autumn, and possibly the winter, a careful look-out should be kept for suspicious grouse, for it is these which require removal particularly. 


\section{CHAPTER VII}

GENERAL CONSIDERATIONS

WE have shown in the preceding account that in the grouse dead of the disease there occurs constantly in the diseased lungs and liver a species of bacilli in a definite distribution; that this species grown on and in various media shows definite characters; and that with the cultures of the bacilli a definite acute fatal disease is produced in mice and guinea-pigs, in buntings, yellow-ammers, and sparrows; that the disease thus produced in the buntings and other birds, in its pathological appearances, compares in a remarkable degree with the disease found in the grouse; and that, further, the disease in the bunting can be set up, not only by subcutaneous inoculation, but also can be communicated directly from a bird ill with the disease to a healthy bird; and that, lastly, in the animals infected with cultures of the bacilli, these latter are found again in definite distribution. All these facts justify us in saying that this species of microbe occurring in the diseased 
lung and liver of grouse dead of the grouse disease is the causa causans of the disease. It will no doubt be argued that the chain of evidence as to this particular microbe being the cause of the grouse disease is in so far incomplete as no infection of normal grouse has been carried out with it. The force of this argument I fully admit, and I have endeavoured to meet it; but owing to the difficulty which I encountered in getting permission to inoculate with the microbe young grouse on the moors on which no grouse disease prevails, all my endeavours have failed. It is clear that such experiments must be carried out on moors on which no grouse disease exists, for if the experiments were made in a locality where grouse disease occurs, the objection could with justice be raised that accidental or natural infection of the experimented grouse was not excluded. I do not think that it would be very difficult to separate a few young grouse - although grouse do not easily bear seclusion in a cage-before they go for cover, and to inoculate them. But this would require my going to and staying at the moors, and getting permission to do it, an expensive experiment which I am not in a position to carry out. But I tried to meet the difficulty in a different way, viz. by getting eggs of grouse and having them hatched by hens. With 
very great difficulty I succeeded, with the kind help of the Editor of the Field, in getting together about two dozen eggs that could be tried. I received more than that from many gentlemen who responded to my appeal; but owing to the insufficient care with which the eggs had been packed, and the great injury they received on the long journey south, they were quite unfit for experiment.

Out of the two dozen eggs thus obtained and subjected to hatching ${ }^{1}$ only one yielded a bird, but unfortunately, also, this expired a few hours after appearing. I was therefore reluctantly forced to abandon the attempt of carrying my experiments further. But, notwithstanding the absence of this last link in the chain of evidence, I think I may say that there can be little doubt that the bacillus which I described is the real cause of the disease,-its constant and definite presence and distribution in the diseased lung and liver of the grouse dead of the disease, its virulence on buntings and other birds, the character of the disease produced in the birds and the transmission by the air from bunting to bunting, with the same distribution of the microbe in the latter, prove to my mind conclusively that we are dealing with a bacillus

1 Messrs. Woodin and Aldous deserve my grateful acknowledgment for having placed at my disposal their farm, near East Grinstead, Surrey, for purposes of this experiment. 
which is not only accidentally present in the grouse disease, and which most certainly is not of the nature of a harmless saprophyte, but which possesses a definite causative relation to it.

This bacillus belongs to a group of pathogenic bacilli which have certain important characters in common, but which, owing to certain other important distinguishing characters, both in culture and in physiological action, represent several definite species. This group of bacilli comprises :

The bacillus of fowl cholera, the bacillus of fowl enteritis, the bacillus of duck cholera, the bacillus of the Middlesbrough pneumonia, the ærobic bacillus of malignant œdema described by me, the bacillus of Frettchenseuche, the bacillus of Wildseuche, the bacillus of swine-plague of the Germans, the bacillus of English swine-fever and American hog cholera. All these bacilli are morphologically similar; they are oval in shape, single, but chiefly in dumb-bells; amongst them are rods and even cylinders, single or in dumb-bells, some motile, others non-motile; they are ærobic, they do not liquefy the gelatine, they grow in a similar but not identical way in gelatine, in plate, streak, and stab culture, but they show considerable and definite differences as regards rapidity of growth; they produce in rodents an acute fatal disease which, generally speaking, partakes of the 
character of a hæmorrhagic septicæmia, but there are well-pronounced differences as regards the duration of the disease and as regards the species of rodents and birds that are susceptible or insusceptible to the different species of these bacilli. 


\section{CHAPTER VIII}

\section{A PATHOGENIC BACILLUS RESEMBLING THE BACILLUS}

OF THE GROUSE DISEASE

IN the course of the spring and summer of I89I I found in garden earth, in very large numbers, a species of bacilli which have a powerful virulent action on rodents, and which, when introduced into them subcutaneously, produce fatal infection; their action on the mouse, and to a certain lesser extent on the guineapig, is not dissimilar to that of the grouse bacilli. In cultivations in various media the bacillus presents a great many points of resemblance with the bacillus of grouse disease, for which it might be easily mistaken; but the two microbes are two distinct species, and therefore the occurrence in numbers of the pathogenic bacillus in earth-whether it also occurs in earth on the moors I am unable to say, I got it from garden earth that had been manured within recent timescannot be brought in any relationship to the bacillus of grouse disease. In order to obviate such a relationship between the two species of bacilli being 
even suspected, I will fully describe this bacillus and its action.

It is well known that recently manured garden earth contains the anærobic motile spore-bearing bacillus of Koch, which, inoculated into guinea-pigs subcutaneously, produces acute malignant œdema, malodorous, rapidly spreading, and leading to gangrene of the muscular and cutaneous tissues, and to the death of the animal in from 20 to 30 hours. This anærobic bacillus of malignant œdema grows in a characteristic and typical way in the depth of grapesugar gelatine and Agar, and produces, as it grows, numerous gas bubbles; it liquefies the gelatine, and forms chains or threads of various lengths.

Desirous of obtaining this classical bacillus of Koch's malignant œdema, I introduced in the customary manner subcutaneously into the groin of the guineapig a small quantity of recently manured garden earth. Next day the animal showed extensive œdema in the groin, on the abdomen, and thorax, was very shaky on its legs, and died in less than thirty-six hours. The sanguineous œdematous fluid was crowded with motile short bacilli, a few longish chains and threads amongst them, also a few that contained the oval spores characteristic of the anærobic malignant-œdema bacillus (Fig. 24). Cultivations were made in ordinary nutrient gelatine and in grape-sugar gelatine, and it was found 
that the very great majority of the bacilli present in the œdema fluid belonged to a species of short motile bacilli which grow best ærobically, i.e. on the surface of the gelatine, which do not at any time liquefy the gelatine, and which do not form spores. By putting a drop of the above œdema fluid into about Io cc. of sterile salt solution, and, after shaking, making from this mixture, with one platinum loop, a gelatine-plate cultivation and cultivations in the depth and on the slanting surface of ordinary gelatine or of sugar gelatine, innumerable colonies of the same species of ærobic, non-liquefying, non-spore-bearing bacilli were obtained. Out of six tubes containing grape-sugar gelatine, inoculated from the salt mixture, only in one tube in the depth did a colony of the classical liquefying œdema bacillus come up, so that the number of these, as compared with those of the ærobic nonliquefying bacilli, was insignificant.

A drop of the œdema fluid inoculated into guineapigs, rabbits, and mice produced, in the first two species of animals, the same malignant œdema, the œdema fluid again malodorous and crowded with the ærobic non-liquefying short bacilli (Figs. 23 and 24). In mice no œdema occurred at the seat of the inoculation, just as in the classical malignant œdema. The heart's blood of the guinea-pigs, rabbits, or mice, dead from the classical malignant œdema or from our 
œdema, contains only a few bacilli; the spleen in the guinea-pig and rabbit is not enlarged, in the mouse only slightly so, and contains few of the bacilli. In all these respects there is, therefore, a complete analogy between the classical malignant œdema and the one under consideration.

Cultivations injected in sufficient quantities subcutaneously in guinea-pigs and mice and rabbits produce the same fatal disease as the garden earth, and there exists also in this respect an analogy between the classical malignant odema and our disease, since it is well known through Gaffky and others that in order to produce a fatal result with cultivations of the classical œdema bacillus, large doses must be injected. There is, however, a slight difference between the two diseases consisting in this, that in the classical malignant œdema the odema is generally more extensive, more distinctly malodorous, and more clearly associated with gangrene of the adjacent muscle than in our disease, though also in this latter the changes are sometimes very severe and not inferior in degree to those in the classical malignant œdema.

Owing to the bacillus in our malignant odema growing well on the surface of ordinary nutrient gelatine, cultures of the bacillus can very easily be obtained from the œdema fluid. These cultures 
in gelatine plate cultivations, in gelatine streak, stab, and shaken cultures, on and in Agar, on potato and in broth, so much resemble the cultures of the grouse bacillus that unless the two cultures are simultaneously inspected and compared one with the other it is extremely difficult to distinguish them. The character of the cultures of the grouse bacillus having been already described in previous chapters, it is unnecessary to state them again.

Making cultivations of the two species at the same time and keeping them under exactly the same conditions, the differences noticed may be stated thus: (I) In plate cultivations of both these bacilli, it is found that the superficially situated colonies of the grouse bacillus are more rapid in appearing and more rapid in spreading, they are flatter, their edges thinner than in those made of our ærobic œdema bacillus; (2) in gelatine streak culture the band formed in the line of inoculation is broader, flatter, and thinner, and grows quicker in the case of the grouse bacillus; (3) in stab culture the plate-like growth on the upper free end of the stab is broader, thinner, and spreads out more; (4) in broth the turbidity of the fluid and the granular precipitate is greater and more copious in the grouse bacillus.

In shaken gelatine cultures in both cases numerous 
flat gas bubbles appear all through the gelatine, one in connection with each individual colony, except in the superficial layers; the gas bubbles situated in the depth remaining longest; in respect of these gas bubbles no difference is noticeable between the two species. On potato the difference noticeable between the two microbes is this, that the œdema bacillus grows faster and spreads out quicker over the surface of the potato than the grouse bacillus, the former producing a more brownish growth than the latter.

As regards the morphological appearances, the gelatine and Agar cultures of our malignant œdema bacillus, examined fresh, show more motile forms than the cultures of the grouse bacillus, and the individual bacilli are distinctly longer and more cylindrical-both in the animal and in the culturein the case of our malignant œdema than in the case of the grouse bacillus; this is a definite and constant difference easily detected.

Measurements of the bacilli of our malignant œdema in dried and stained cover-glass specimens, show :-

In the œdematous fluid of the guinea-pig:

Thickness $0.7 \mu$

The majority in length $0.8-1.2 \mu$

$$
\begin{aligned}
& \text { Some " }, \quad \text { ז.6-2.4 } \mu \\
& \text { Few up to . . } 2.4 \mu \text { long. }
\end{aligned}
$$


In specimens made of broth and Agar cultures :

The majority in length $1.6-2.4 \mu$.

In specimens from gelatine cultures :

Length of the majority $0.8 \mu$.

By comparing these figures with those given of the bacilli in grouse disease it will be seen that our malignant œdema bacilli are distinctly longer than those of the grouse disease.

Another difference is the distribution of the bacilli in the animals (mice and guinea-pigs) infected: the grouse bacillus is present in the blood and spleen in greater abundance than our malignant œdema bacillus; the odematous swelling in the guinea-pig after inoculation with œdematous fluid or cultures of our œdema bacillus is always more pronounced than after inoculation with the cultures of the grouse bacillus. Rabbits are susceptible to our malignant œdema bacillus, but insusceptible to the grouse bacillus.

If guinea-pigs or rabbits are inoculated with a drop of the cedema fluid, or with a good dose of our malignant odema bacillus, the animals are found ill and quiet as early as I 2 hours after inoculation; they are found dying or dead in from 20 to 36 hours with considerable œdema and hæmorrhage in the groin, on the abdomen, and chest. Sections made through the skin, the subcutaneous and muscular 
tissues, show the tissue of the cutis and 'the subcutaneous tissue crowded with the bacilli-the bacilli either uniformly distributed or aggregated in clumps ; owing to the odematous condition, the interfascicular spaces are distended and contain numerous leucocytes; the adjacent muscular fibres are œdematous, disintegrating, and in the lymph-spaces of the connective tissue between the muscular fibres are crowds of the bacilli. Now after inoculation with the grouse bacillus such a state is not noticed. The leucocytes of the adematous subcutaneous tissue just mentioned, in very acute cases, include the bacilli, some only one or two, others are crowded with them (see Fig. 27).

If for inoculation of a guinea-pig a small dose of a cultivation of the malignant odema bacillus is used, say one or two minims of a recent broth culture, the animal does not die; it is ill after 24 hours, and shows an œdematous swelling, but this is not by any means so large as after a large dose. The animal, as just stated, does not die, and the soft œdematous swelling in a few days decreases, becomes firm, and forms a solid cord or band in the subcutaneous tissue; this firm swelling in some cases leads to necrosis and sloughing of the skin with complete healing, or it gradually, during a week or fortnight, becomes smaller and smaller till it 
entirely disappears; rarely, the animal dies between the end of the first and the end of the second week. If the tumour of such a "chronic" animal is hardened and examined in sections, stained first with rubin and then with methyl blue, one finds numerous bacilli in the tissue of the tumour. That they are living can be shown by the successful cultures made of the fresh tumour tissue. What is, however, remarkable is that while the substance of the leucocytes in the subcutaneous tissue at and about the seat of inoculation in the acute fatal cases encloses the bacilli abundantly, there is nothing of the sort to be seen in the leucocytes of the "chronic" tumour. This is rather contrary to what we ought to expect if the theory of phagocytosis (Metschnikoff), i.e. the swallowing and destruction of the bacilli by the leucocytes, were applicable here; for it would be serving no object if the leucocytes of the œdematous tissue in the acute cases were destroying them, seeing that the tissue in general is flooded with the bacilli, and the animals die rapidly; whereas in the "chronic" cases, if the resistance of the animal were due to a salutary part played by the leucocytes, to wit, acting as phagocytes : taking up and destroying the bacilli and thus preserving the life of the animal, we should expect to find at least some of the leucocytes enclosing the bacilli; but evidence 
of this kind is altogether absent in the chronic cases.

Just as in the case of the grouse bacillus, so also with our malignant œdema bacillus; animals that have passed through a mild attack and recover withstand a second inoculation with a fatal dose.

As has been mentioned in a previous chapter, the bacillus of grouse disease does not form spores; the same holds good for the bacillus of our malignant œdema. Complete drying kills the latter; heating cultures to $60^{\circ} \mathrm{C}$. for five minutes kills all life; no further sub-cultures can be made. It is on these grounds easily possible to separate in garden earth the classical (Koch) anærobic spore-bearing bacillus from our ærobic non-spore-bearing bacillus. By exposing garden earth mixed with a little sterile salt solution in a test-tube to a temperature of $70-75^{\circ} \mathrm{C}$. for five minutes, the spores of the classical anærobic bacillus are preserved, while our bacillus is killed; with such garden earth I inoculated guinea-pigs subcutaneously, and now produced the classical malignant œdema, the œedema fluid crowded with the classical anærobic spore-bearing bacilli; by cultivation this œedema fluid can be shown to be free of our ærobic bacillus.

In order to ascertain further differences between the bacillus of grouse disease and our ærobic bacillus of malignant œdema, I inoculated yellow-ammers 
with recent broth cultures (one to two minims) of the latter. The birds were found dead or dying before 24 hours were over, the heart's blood and the blood juice of the hyperæmic lungs containing but comparatively few of the bacilli, in strong contrast to the condition described of the behaviour of the bacillus of grouse disease.

An important difference, however, exists between the action on pigeons of our ærobic œdema bacillus and that of the grouse bacillus. As has been stated in a previous chapter, pigeons are refractory to even large doses of virulent broth culture of the grouse bacillus, but on injection with broth culture of our malignant œdema bacillus they are anything but refractory. On the day following the subcutaneous inoculation with 5 minims of a recent broth culture of our malignant œdema bacillus, the pigeons were found distinctly ill, quiet, off their food, when made to move they were seen to be very shaky on their legs, and they were very unwilling to move; this condition lasted for several days, but eventually they recovered.

A very remarkable antagonistic action exists between the two bacilli if they are injected at the same time into the same mice. Recent (24-4S hours old) broth cultures of either of them, injected separately under the skin of the back of white mice, in doses varying between 2 and 5 minims, produce illness and 
death in one to three days in the great majority of the animals; they die with the same symptoms and with very similar post-mortem appearances: no œdema at the seat of the inoculation, congestion of both lungs, the spleen slightly enlarged; the heart's blood and the lungs and the spleen contain the bacilli abundantly. Now I inoculated mice with broth culture ( 24 hours old) of our odema bacillus, each animal receiving $2-5$ minims; other mice were inoculated at the same time and in the same doses with broth culture ( 24 hours old) of the grouse bacillus, and a third lot were inoculated at the same time with a mixture in equal parts of the very same broth cultures of our bacillus of malignant œdema and of the grouse disease. The result was very remarkable. Three such series were made: in each series two mice being used for each experiment. In series one, both mice inoculated with the malignant odema bacillus were ill and weakly, one died before the second day was half over, the other survived; the two mice done with the grouse culture both died before the second day was over; and of the two mice done with the mixture, one died on the sixth day, the other survived. In series two, both the mice done with the malignant œdema bacillus died; one of the two mice done with the grouse culture died on the second day, the other, though very ill, recovered; of the two mice done with 
the mixture, one died on the fifth day, the other survived. In series three, both mice done with the culture of malignant œedema were found dead on the second day with abundant bacilli of malignant œdema in the blood; both mice done with the culture of the grouse bacillus were found dead on the second day with abundant bacilli of the grouse disease in the blood; and both mice done with the mixture were alive and well on the seventh day. Now in this last series the amount of the mixture injected in the last two mice was double the amount of the culture of malignant œdema and of grouse separately, and although the two mice that were inoculated with the same amount of malignant œdema alone, as also the two mice inoculated with the same amount of grouse culture alone, succumbed, yet the two mice inoculated with the mixture, that is, with double the amount, survived. Clearly there is then a decided antagonism within the body of the mouse between the two species, by which the action of the one is neutralised by that of the other. If the two species were the same, then the two mice done with the mixture, having received a double dose, ought to have succumbed so much more readily, whereas on the contrary they remained alive; it follows from this that the two microbes are two totally different species. This conclusion must, therefore, remove all doubt as to any close relation 
existing between our bacillus of malignant œedema occurring in earth and the bacillus of grouse disease; and it is obvious that notwithstanding certain striking cultural similarities between the two species, no valid reason exists to accuse our bacillus of earth, i.e. that of our malignant œdema, as causally connected with the grouse disease. 


\section{PART II}

FOWL CHOLERA, FOWL ENTERITIS, AND "CRAMPS" IN YOUNG PHEASANTS 



\title{
CHAPTER IX
}

\author{
FOIVL CHOLERA
}

DURING the early part of I 889 I became informed of the existence of an acute and fatal epidemic disease amongst fowls at a poultry-farm in Kent, which was represented to me as being chicken cholera, and as I had not till then come across or heard of the existence of chicken cholera in England, I was anxious to obtain material from that farm. The fowls which I received were carefully examined and used for microscopic examination, for cultivation, and for animal experiment, and it very soon became evident that the disease with which these fowls had been affected was clearly distinct from chicken cholera; it was a disease which, though similar to, was in many important points different from chicken cholera; I have called it fowl enteritis. In order that the reader may be in a position to correctly understand these points of similarity and difference, I will first describe the nature of chicken cholera, known so well and so com- 
pletely by the researches of Perroncito, Toussaint, Pasteur, Kitt, and others.

The disease fowl cholera or chicken cholera, rife in France and Germany, is an acute infectious disease, rapidly fatal, and producing great devastations amongst fowls. After an incubation of from 16 or I 8 hours to a day or a day and a half, the fowls show the first symptoms of the disease-diarrhœa of thin, greenish, mucoid evacuations, unwillingness to move, and quietness; they refuse food; they soon become drowsy and somnolent, and this rapidly increasing, leads to death in from 24 to 36 or 48 hours. On post-mortem examination the heart is distended and filled with blood that rapidly clots, the lungs, liver, spleen, intestines, and peritoneum are found much congested; in the lungs, liver, and intestine, particularly the duodenum and in the peritoneum, hæmorrhages occur in the form of petechiæ and patch-like blood effusions. The blood of the heart and the general circulation is crowded with minute oval or short rod-like bacteria, known as the bacilli of fowl cholera; their number is generally as great, often greater, than that of the blood-corpuscles (Figs. 32, 33, and 34). In stained preparations the rods show a characteristic deeply stained granule at each end, the middle part of the rod being clear; hence at first sight it appears like a dumb-bell. Some 
of the rods form dumb-bells or even short chains. The size of the single rods in stained preparations is $0.2 \mu$ thickness, the spherical ones $0.2 \mu$ in diameter, the oval and rod-shaped $0.4-0.5 \mu$ in length, some are longer, $0.8 \mu$; there are some which measure I-I.5 $\mu$, but on careful examination it will be found that these are really dumb-bells of rods. ${ }^{1}$

The bacilli are present in large numbers in the blood of all organs, and almost in pure cultivation in the mucoid contents of the intestine.

Inoculation into fowls, pigeons, and rabbits, of a drop of blood or of a drop of a broth culture of the microbe, produces invariably the disease and death in from 20-36 hours under the same symptoms, and the same distribution of the bacilli. In the rabbit, death as a rule occurs within 24 hours, sometimes in as short a time as i 8-20 hours, the blood teeming with the bacilli; hæmorrhages in the liver, the intestine, and peritoneum being generally found. In the rabbit dead of the inoculated disease, the bacilli are oval or rod-like and of the same character as those in the fowl and pigeon, but there are a great many that are almost spherical, far more than in the

1 The size of the bacilli generally given in text - books (Flügge, Baumgarten) is too large. The above figures given by me were ascertained with exact measures. In the illustrations given both by Fliigge and by Baumgarten, the microbe is also represented of too large a size, as is proved by photographs. 
fowl and pigeon. Specimens made of cultivations in broth, on gelatine, or on Agar show, besides the characteristic oval rods, also numerous dumb-bells or chains of four.

If a drop of blood of an animal dead of the disease, or of a recent culture, be examined in the fresh condition, the bacilli are not possessed of active motility, all appearing non-motile or only showing Brownian molecular movement. A trace of a droplet of the heart's blood of a fowl, pigeon, or rabbit dead of the disease, used for a gelatine plate cultivation or rubbed over the slanting surface of nutrient gelatine and kept at $20^{\circ} \mathrm{C}$., yields innumerable colonies. The first indications of the colonies visible to the unaided eye do not appear before 48 hours are over, though with a magnifying-glass they can be just discerned after $3^{6}$ hours. When easily visible to the unaided eye, that is, during the third and fourth days, and even later, they appear as minute dots, greyishyellow in reflected light. Under a lens they are round, sharply outlined, and somewhat prominent, brownish and granular in transmitted light. The colonies growing on the surface are a little larger and have a slightly uneven outline, recognisable as such under a glass, those in the depth remain very small spherical dots (Fig. 35). In gelatine streak culture, after an incubation at $20^{\circ} \mathrm{C}$., there is visible, 
but not before 48 hours, a thin greyish-yellow line of growth which gradually broadens during the next days, its outline rounded and knobbed, but even after a week to a fortnight does not reach in breadth more than a few millimetres. In stab culture the stab becomes marked as a line composed of minute dots, brownish in transmitted light, whitish-yellow in reflected light; at the upper free end of the gelatine is a small rounded plate of growth slightly raised, with irregular outline, and, after a week or ten days, measuring a few millimetres. The gelatine is never liquefied. Alkaline beef-broth peptone incubated at $35-37^{\circ} \mathrm{C}$. becomes slightly turbid by the growth of the microbe in 24 hours; the turbidity increases during the next days, but, even when the maximum development has been reached, is never very pronounced. There is then present a small amount of a greyishwhite powdery precipitate. On the surface of Agar the growth forms a thin, translucent, grey film; on potato sterilised in the steamer, and then kept at $35-37^{\circ} \mathrm{C}$., the growth is slow and remains fairly limited to the region of inoculation, forming a whitish or light brown film spreading slowly in breadth.

As has been mentioned above, a drop of a broth culture $24-48$ hours old, or a drop or two of a salt mixture of the growth taken from a fairly recent gelatine or Agar culture, invariably produces fatal 
infection in fowls, pigeons, and rabbits. And the virulence of the cultures, be they derived from fowl, pigeon, or rabbit, is maintained unimpaired for an unlimited number of sub-cultures in broth, gelatine, or Agar, provided these be used when recent. I have had sub-cultures which had been carried on on gelatine for from several months to over two years; fresh broth sub-cultures started from them proved always virulent. Fowls can be infected not only by subcutaneous injection but also by feeding, but the result is not by any means so certain; since some animals escape infection. As has been mentioned above, the intestinal contents teem with the bacilli, and it is therefore justifiable to assume that under natural conditions the food picked up from the ground by the fowls becomes contaminated with the microbes of the dejecta. Before concluding this description, which is introduced here merely for the sake of comparing this disease with the disease to be described presently, viz. with the fowl enteritis, I wish to mention two further points :-

(I.) The enormous rapidity with which the microbe multiplies in the rabbit. A rabbit of known weight was inoculated with a drop of broth culture containing about 20,000 bacilli; the animal was dead with the typical disease in 20 hours. The number of the bacilli present in the heart's blood was then ascertained, and 
assuming the bacilli to be distributed throughout the blood in a uniform manner, it was calculated that, provided the relation of blood to body weight be about I to I4 or I5, there were present in the total blood over I 200 millions of bacilli, that is to say, that the 20,000 bacilli introduced into the animal had multiplied in 20 hours so as to yield 200 millions, or in other words, that each one of the introduced bacilli had produced a crop of 60,000 bacilli.

(2.) On examining sections of the hardened liver of animals-fowl, pigeon, or rabbit,--dead of the disease, there are always found in each section patches of the liver acini in which the capillary vessels are distended and filled with the bacilli ; the liver cells around being dark, granular, and disintegrating; in the fowl they are filled with fat droplets, but in all parts of the section all the blood-vessels contain crowds of the bacilli, and many of them are present outside the vessels between and around the liver cells (Figs. 38 and 39).

The illustrations which are given in Figs. 32-40 give an accurate representation of the nature of the gelatine cultivations, of the shape and size of the microbe, and of their distribution in the blood and the organs. 


\section{CHAPTER X}

\section{FOWL ENTERITIS-NATURE OF THE DISEASE}

In I $888 \mathrm{Mr}$. W. Cook, well known through his works and writings on fowls and fowl-breeding, had on his poultry-farm, then at Orpington, in Kent, in about two acres of land, a fatal epidemic of fowls by which he lost, between March I 888 and March I889, over 400 birds. The greatest number of birds, nearly 200, died during March and April 1888, occasionally 6 per diem; during the beginning of 1889 the number of fatal cases had decreased considerably-to about 7 per week. Mr. C. Wellington, then at Mr. Cook's farm, was kind enough to supply me, with Mr. Cook's permission, with birds affected with the disease and either dying or recently dead. From these birds I obtained materials with which I commenced experiments on normal fowls and other animals. On making post-mortem examinations the difference between this fowl disease and fowl cholera-for such was the disease considered-was distinct. All the fowls that I received from Mr. Cook's farm, and 
those experimental fowls which I afterwards had occasion to examine, presented the same kind of post-mortem appearances: the heart was full of coagulated blood; this condition of the blood was noticed very soon ( $\frac{1}{2}$-I hour) after death. The lungs in all animals were normal, no congestion, no hæmorrhage; the intestines showed redness in the mucous membrane and in the serous covering, but there was no hæmorrhage anywhere; in the cæcal appendages there was a good deal of mucus, either in the form of flocculi or as continuous yellowish, greyish, or brownish masses; the rectum contained greenishyellow fluid fæcal matter. The spleen was enlarged to twice or thrice its normal size, but its substance was not excessively bloody, since from a cut surface there was not that oozing out of sanguineous fluid commonly observed of a hyperæmic spleen. The liver was somewhat enlarged, soft, and flabby, there were no hæmorrhages; the surface of the liver and of the peritoneum was moist, occasionally there was a small amount of exudation, but no hæmorrhages. Examining the fresh blood, it was at once noticed that though bacteria could be found here and there in each field of the microscope, they were very few and far between, one or two small rods only being met with in one field. Making cover-glass specimens, and, after drying and staining, examining them under 
the microscope, this condition, viz. the relative scarcity of the bacteria in the blood, was confirmed (Fig. 4I). It was at the same time noticed that the bacteria were distinctly thicker and longer than in fowl cholera, they occurred also as single rods or as dumb-bells with rounded ends. This condition, as regards the bacilli in the blood, was uniform in all the fowls that I got from Orpington, as well as in those which were infected in the laboratory from the Orpington fowls.

Thus far it was quite clear from these observations that the Orpington disease differed from fowl cholera: (I) in the absence of hæmorrhagic changes so common in fowls dead of fowl cholera; (2) in the scarcity of the bacteria in the blood; (3) in the difference in size between the bacteria present in the blood of the Orpington fowls and those of the typical fowl cholera. Further differences established were these: the spleen of the Orpington fowls, examined in cover-glass specimens, contained the bacteria somewhat more numerously than the blood (Fig. 42), but very far less numerously than in fowl cholera, and also the bacteria of the spleen of the Orpington fowls were distinctly thicker and longer than those of the fowls dead of fowl cholera.

Measurements of the bacilli of the blood or spleen tissue, or of cultures in dried and stained cover-glass specimens, showed :- 
The thickness of the bacilli $\quad 0.3-0.4 \mu$.

The length of the single bacilli . 0.8-1.6 $\mu$. Some as long as $2 \mu$.

The mucus of the intestine of the Orpington fowls contained the bacteria, just as the mucus of the fowl-cholera fowls, in almost pure cultivation, either as spherical or oval coccus-like organisms, singly or as dumb-bells, or as distinctly cylindrical bacteria (Figs. 43 and 44). Examining in salt solution or broth the fresh bacteria of the blood or spleen of a fowl dead of fowl cholera, or of gelatine cultures of the same, there are no motile bacilli to be met with. The bacteria of the Orpington fowls are also devoid of motility, for in no preparation, either of the blood or spleen, or of gelatine or other cultivations, did the bacilli reveal anything like motility. As has been already mentioned, inoculating subcutaneously with the blood or spleen-juice of a fowl dead of fowl cholera, fowls, pigeons, or rabbits, invariably a fatal result is produced in one or two days, or even less, with the characteristic symptoms and post-mortem appearances of fowl cholera. Experiments of the same character were therefore instituted with the blood and spleen tissue of the Orpington fowls, but the result was totally distinct from that obtained in fowl cholera, for the pigeons proved quite insusceptible; 
so were also the rabbits that had been inoculated with the blood or spleen of the Orpington fowls. All pigeons and rabbits remained alive and well. The fowls that were so inoculated remained well for the first four or five days, then they left off feeding, became quiet, and showed diarrhœa of greenish-yellow evacuations; a day or two later they were found dead, but in no case did they show the somnolence characteristic of fowl cholera. I experimented on a large number of fowls with blood and spleen tissue of the Orpington fowls, or of fowls that succumbed after inoculation from the Orpington fowls, also on a very large number of fowls that I inoculated with cultures of the blood or spleen tissue, dead from the natural disease or after inoculation, and in no single instance have I found any difference in the results, in no single instance have I seen any such rapid course as characterises fowl cholera. The earliest date at which the animals showed illness was the fourth day, and this, amongst a series of experiments comprising several dozens of fowls, occurred only once or twice. Most animals remained well for the first four days at any rate. Death in no single instance occurred before the sixth day, in the majority of instances during the seventh day, occasionally the eighth or ninth day. On account of the enteric disturbance being a prominent symptom, and to distinguish the 
disease from fowl cholera, I have called it fowl enteritis. I have described the nature and character of the disease in the Centralblatt fiir Bacteriologie und Parasitenkunde, I889, v. 2 I and following; and although the different size and scarcity of the bacteria in the blood in the Orpington fowls was clearly pointed out, as also the difference in the appearances at the post-mortem examination in fowl enteritis and fowl cholera; the remarkable and striking fact that pigeons and rabbits were found insusceptible to the disease, and other differences, were clearly stated, yet some critics ventured to offer the opinion that the Orpington disease, i.e. the fowl enteritis, is not sufficiently well differentiated from fowl cholera to be declared not to be such. To this I can only say,-and in this I am sure every pathologist and bacteriologist who has any personal knowledge of fowl cholera will agree with me,- that those critics either are not sufficiently, certainly not directly, acquainted with fowl cholera, or they have not read my statements as regards the Orpington disease, or both.

Other equally striking differences are noticed between the two diseases if the cultures obtained from the Orpington fowls are studied. 


\section{CHAPTER XI}

CULTIVATION OF THE BACILLUS OF FOWL ENTERITIS

A DROPLET of blood taken from the heart and used for a gelatine plate cultivation, or rubbed over the slanting surface of gelatine, yields always a pure cultivation of colonies of the bacilli of fowl enteritis. The number of colonies of a droplet of blood of a fowl dead of fowl enteritis compared with the number of colonies of a droplet of blood of a fowl dead of fowl cholera, as may be expected from what has been said above, is strikingly different. This difference can be made very conspicuous by diluting the blood first and then making cultivations. A measured drop of blood-one minim or one division of a Pravaz syringe-of a fowl dead of fowl cholera is added to Io cc. of sterilised (boiled) salt solution, and well mixed; from this a droplet is taken by means of the platinum loop, and with it a gelatine tube is inoculated, and from it a plate cultivation is made; or it is rubbed over the surface of the slanting gelatine contained in a tube. The number of colonies making their appearance in 
such cultivations on incubation is still very considerable. But if on exactly the same lines plate cultivations or slanting gelatine tube cultivations be made of the blood of a fowl dead of fowl enteritis, the number of colonies is extremely limited,- - some tubes remain free of colonies, others contain only a very limited number.

The number of colonies that make their appearance when using a small particle of the spleen tissue in fowl enteritis is, as might be expected from what has been said in the preceding chapter, considerably greater than when using the heart's blood. The character of the colonies and the rate of growth are different in the two diseases. In the case of the fowl enteritis, the first signs of the colonies are noticed with a glass very distinctly after 24 hours' growth as greyish translucent dots. During the second and third days the colonies situated on the surface rapidly enlarge into greyish-white translucent discs with thinner angular margin; they remain small spherical dots when situated in the depth. In transmitted light the superficial as well as the deep colonies are brownish, the latter of course more so than the former. The superficial colonies grow steadily in breadth, become more angular, are large and thin as compared with those of fowl cholera (Fig. 45), and do not at any time look yellowish-white like the latter. The chief 
difference, however, is shown in the rate of growth: the colonies of the fowl-cholera bacillus grow conspicuously slower and remain smaller than those of the fowl-enteritis bacillus. I have repeatedly had occasion to make comparative experiments; in this I was ably assisted by Dr. Wood, of Melbourne, while working in my laboratory: cultivations of the fowlcholera bacillus and of the fowl-enteritis bacillus were made at the same time under the same conditions in the same stock of nutrient gelatine, kept then under precisely the same conditions, and always, without exception, the differences described above in the aspect, and particularly the rate of growth, of the two microbes were conspicuous and constant. The time at which the colonies of the fowl-enteritis bacillus appeared, and the rate with which they grew afterwards, the first much shorter, the latter very much greater, were appreciably different from those of the colonies of the fowl-cholera bacillus.

In sub-cultures in gelatine, in streak and stab, the growth is distinct even during the second day: in the streak culture a greyish-white flat band appears, which gradually broadens during the following days; its margin is irregularly knobbed and sinuous. After about two weeks the band is 3-5 millimetres broad, but it remains flat; in reflected light it looks whitish, in transmitted light, light brown. In stab culture, during 
the second and third days, the line of inoculation becomes visible as a greyish-white line, which under a glass is seen to be made up of spherical dots; on further incubation these enlarge, but remain always separated; on the upper free end of the stab the growth is a thin translucent greyish-white film with crenate margin.

The differences in the rapidity of the growth in streak, stab, and shake culture shown by the bacillus of fowl cholera and of fowl enteritis are very marked, that of the latter being considerably more rapid than that of the former.

The gelatine is never liquefied by the growth.

In faintly alkaline broth kept at $35-37^{\circ} \mathrm{C}$. the bacillus of fowl enteritis grows rapidly, forming uniform turbidity in twenty-four hours; the turbidity gradually increases, and, compared with that produced by the fowl-cholera bacillus, is always at the same distance of time markedly greater; there is also a greater amount of whitish precipitate in the broth cultures of the bacillus of fowl enteritis than in those of the bacillus of fowl cholera; there is never any formation of anything like a pellicle. Broth cultures of the bacillus of fowl enteritis kept at $35-37^{\circ} \mathrm{C}$. begin to get clearer in the superficial layers by the end of the week. On nutrient Agar incubated at $35-37^{\circ}$ C. the bacillus of fowl enteritis forms a whitish-grey 
translucent film with sinuous margin; after about a weck it has spread almost over the-whole surface of the Agar, but it remains a thin film.

Equally marked differences between the bacillus of fowl cholera and fowl enteritis are shown by their behaviour on potato; while the bacillus of fowl cholera shows distinct growth on boiled or steamed potato, as has been described in a former chapter, the bacillus of fowl enteritis practically does not grow on potatoeven after a week at $35-37^{\circ} \mathrm{C}$. incubation the point of inoculation is only just perceptible as a whitish patch ; of anything like an appreciable amount of growth nothing is to be made out.

As regards aspect and size of the bacilli of fowl enteritis there is not much to be added to what has been said already; in all media the bacilli are either oval or rod- or cylindrical-shaped organisms, either single or in dumb-bells, their ends rounded, and when examined fresh in salt solution or in broth, never showing motility. Their length, however, varies somewhat in gelatine and Agar cultures. Cover-glass impressionpreparations of gelatine plate cultivations, dried and stained, show that in some colonies the bacilli are conspicuously more cylindrical than in others. Fig. 48 is a photograph taken from an impression specimen of a colony on the surface of a gelatine plate, and here the bacilli are all conspicuously cylindrical, so much so 
that on account of their uniformity I was at first inclined to doubt their genuineness, but I have, by repeated examination and by sub-cultures, convinced myself of their being genuine fowl enteritis bacilli. Some colonies, however, show many of the bacilli shorter and not of such uniformity in length (Fig. 49). A comparison of photographs made of impression specimens of the colonies of the bacillus of fowl cholera and of the bacillus of fowl enteritis shows the difference in thickness and length of the two species very conspicuously (Figs. 36 and 48). In broth cultures of the bacillus of fowl enteritis there are many bacilli which are cylindrical in shape, some in chains, and some even forming filaments.

It has been mentioned in Chapter IX. that the mucus in the intestine in fowl enteritis is crowded with the bacilli ; cover-glass specimens show this suffciently well (see Figs. 43 and 44). This fact is confirmed by cultivation. The intestine-best the cæcal appendages-having been opened with sterile scissors, a particle of the mucus is removed with a platinum loop and placed in a test-tube containing a few cubic centimetres of sterilised salt solution or beef broth; it is then well shaken. From the mixture, by means of the platinum loop, inoculations are made of gelatine, used either for plate cultivations or the droplet is rubbed over the surface of the slanting gelatine and 
then incubated. Large numbers of colonies of the bacillus of fowl enteritis make their appearance with scarcely any other contaminating bacteria: I have in this way obtained, directly from the intestinal mucus, pure cultivations with numerous colonies on the slanting surface of gelatine.

Dr. F. H. Andrewes has in my laboratory ascertained that various species of microbes, which, when grown on the ordinary nutritive media, occur chiefly as oval or short rod-like organisms, - of swine fever, Middlesbrough pneumonia, grouse disease, - when grown on similar media, but to which previously had been added Stedman's sea salt, to the amount of five per cent, always form filamentous and thread-like bacilli, either straight or wavy, singly or apparently branched ; and when cultivated back on the ordinary media again produce growths of the oval or short rod-like forms. Dr. Wood repeated for me these experiments with regard to the fowl cholera and fowl enteritis bacilli, and he ascertained as a uniform result that while the bacillus of fowl cholera does not grow in the salted media (gelatine, Agar, beef broth), the bacillus of fowl enteritis grows well therein, forming peculiar curved filaments of various lengths, sometimes very considerable, the threads being single or apparently branched: but this latter may be only apparently so, because a matting together 
and a consequent appearance of branching cannot be easily excluded.

I have also compared the two bacilli in their behaviour in gelatine to which previously a trace of litmus had been added-sufficient to render the gelatine of a slight bluish tint. While the bacillus of fowl enteritis decolorises the litmus gelatine markedly at and around the growth, the bacillus of fowl cholera does this only very slightly indeed. (As is the case with other organisms, tested by various observers, this method of growing microbes in litmus-gelatine, since its introduction by Buchner, is a common method of cultivation for the sake of differentiation.) The decoloration produced also by the bacillus of fowl enteritis is more rapid and more pronounced near the surface than in the depth.

From all these observations, then, we are in a position to affirm that also in respect of the character of the cultivations in various media the bacillus of fowl enteritis is a distinct species, well differentiated from the bacillus of fowl cholera. 


\section{CHAPTER XII}

EXPERIMENTS ON FOWLS

I. I HAVE already mentioned in Chapter IX. that several experiments of subcutaneous inoculation of fowls were carried out with blood or spleen tissue of the Orpington fowls, dead naturally from fowl enteritis. Altogether eight fowls were thus inoculated; four of these were ill on the 5 th-6th, four on the 6th-7th day, being quiet and off their food and having diarrhœa; five died on the 7 th, two on the 8th, and one on the 9th day. The post-mortem appearances were in all these animals the same, and such as have been already stated. The bacilli were demonstrated in every one of these in cover-glass specimens, and in cultivations of the heart's blood, the spleen tissue, and the intestinal mucus.

2. Two fowls were inoculated with the blood or spleen tissue of one of the above experimental fowls that had died of the disease after inoculation; the result was exactly the same as above.

3. Inoculations of fowls were also made with 
cultivations derived from the blood of the Orpingiton fowls. From a broth culture (first sub-culture), after twenty-four hours' incubation, about Io minims were inoculated subcutaneously into each of the two fowls. On the 6th day both animals were found quiet and dull, off their food, and suffering from diarrhoea. On the 7 th day both were found dead with the characteristic post-mortem appearances; cover-glass specimens and cultivations of the heart's blood, spleen tissue, and intestinal mucus yielded abundant evidence of the presence of the bacilli.

4. From a second broth sub-culture twenty-four hours old, which had been established from a previous gelatine sub-culture, two fowls were inoculated. Both showed the first signs of illness on the 6th day, being quiet, off their food, and affected with diarrhœa. One of these fowls was found dead on the 7 th day, with the typical appearances, and the bacilli in the blood, spleen, and intestinal mucus. The second one was still quiet and had diarrhœa on the 8th day. On the $9^{\text {th }}$ day it still had diarrhœa, but appeared a little more lively and was seen feeding. On the ioth day the diarrhœa was less, and the fowl was distinctly better. The diarrhœa gradually subsided, and at about the I Sth-2oth day the fowl seemed to have perfectly recovered.

Two other fowls were inoculated subcutaneously 
with several minims of a broth sub-culture, twenty-four hours old. Both birds showed the first signs of illness on the 5th day; they were quiet, off their food, and had diarrhœa. The condition was the same on the 6 th and 7 th days; on the 8 th day the diarrhœa was less, the birds were livelier, moved about, and fed also a little; they gradually recovered from the diarrhœa, and by the end of the third week had seemingly quite recovered.

[I shall have occasion to refer further below to a large number of inoculation experiments on fowls made with broth cultures, but as they were made with broth cultures previously exposed to certain higher degrees of temperature, with the object of attenuation, they will be described in connection with the protective inoculations.]

In order to see whether the infection of fowls can be produced by feeding, the following experiments were instituted :

5. Some of the intestinal contents of a fowl that had died of the disease after inoculation with blood and spleen tissue of an Orpington fowl, were poured down the throat of two fowls. One of these birds showed the first signs of illness-viz. slight diarrhoea on the 6th day, on the $7^{\text {th }}$ day the diarrhœa was profuse, the bird was quiet, dull, and off its food. This animal was found dead on the 8th day. On post- 
mortem examination the typical appearances were found. The blood, spleen tissue, and intestinal mucus contained the bacilli ; cover-glass specimens and cultivations proved that the intestinal mucus was almost a pure culture of the bacilli of fowl enteritis. The second fowl remained lively and showed no diarrhœa. After the lapse of eighteen days since the feeding, this fowl was inoculated subcutaneously with a particle of a first sub-culture on gelatine. On the $5^{\text {th }}$ day the bird was quiet and had diarrhœa, on the 6th the diarrhœa was profuse, and on the 7 th the fowl was found dead with the characteristic appearances and the typical distribution in its body of the bacilli of fowl enteritis.

6. Two fowls were fed with a mixture, prepared by mixing broth, Agar, and gelatine sub-cultures, the two former about one month old, the last about six weeks old. By the 7 th day no sign of illness was noticed in these fowls. They were fed again with a gelatine sub-culture, eleven days old, but no illness was noticed by the 8 th day.

From this series it follows that by feeding with the intestinal contents the disease is producible, but with cultures the experiment has not yielded positive results; it ought to be noted, however, that the cultures used in Experiment 6 were of considerable age, and it must be added that the second feeding 
of the same fowls with gelatine sub-culture, eleven days old, proved without success, although the same gelatine sub-culture when inoculated subcutaneously (Sub-experiment 5) proved virulent. We conclude, therefore, that although some bacilli-e.g. of the intestinal mucus-when introduced by ingestion, escape the action of the stomach and prove capable of infection (Experiment 5 ), this is the case only to a limited degree.

\section{Experiments on other Animals}

7. Pigeons, notoriously susceptible to fowl cholera, are quite refractory to fowl enteritis; in no single instance have I been able to produce illness after inoculation with large doses of virulent material; that is, material which proved virulent for fowls, even in small doses.

8. As is well known, rabbits are extremely susceptible to fowl cholera; but to fowl enteritis their behaviour is altogether different. A drop of blood of a fowl dead of fowl cholera, or a drop of a recent broth sub-culture of the bacillus of fowl cholera, injected subcutaneously into a rabbit, produces infection and death in from 20 to 30 hours without fail. Rabbits were injected subcutaneously with 5-IO minims of blood and spleen tissue of the Orpington fowls, as also of 
fowls dead after inoculation from these fowls, but no result followed-the rabbits remained alive.

Rabbits were then injected subcutaneously with broth sub-culture of the bacillus of fowl enteritis. Altogether six rabbits were inoculated, each receiving $\frac{1}{2}$ to I Pravaz syringeful of a culture twenty-four hours old; the same culture was tested on fowls and proved virulent. Of the six rabbits five remained well, the sixth was found dead on the fifth day. On post-mortem examination both lungs were found much congested, the spleen was dark and very slightly (?) enlarged, the liver was slightly congested, no apparent change in the intestine. Cultivations on gelatine were made of the heart's blood and incubated. A very limited number of colonies made their appearance. Three drops of blood yielded altogether thirty-four colonies, all of them typical colonies of the bacillus of fowl enteritis.

From this it is quite clear that fowl enteritis and fowl cholera are two different diseases; further proof will be furnished later on by showing the behaviour towards fowl cholera of rabbits that survived a first inoculation with fowl enteritis.

9. Guinea-pigs were also inoculated by injecting them subcutaneously, some with blood and spleen tissue of a fowl dead of fowl enteritis, others with active broth culture of the bacillus of fowl enteritis, 
but no result was obtained; the animals remained alive and well.

IO. Mice (two in number) were inoculated under the skin of the back with a large dose of a gelatine culture of the heart's blood of an Orpington fowl. Both animals were found ill after twenty-four hours; coat rough, "lumpy," breathing rapid. After fortyeight hours the animals were worse, very quiet, eyes closed. After three days they were so bad that they looked dying. One died during the day. On postmortem examination the liver was found congested, the spleen dark and distinctly enlarged; no other change. Cultivations were made of the heart's blood and the spleen juice, but on incubation only a few colonies of a liquefying coccus were found, no colonies of the bacillus of fowl enteritis. The second mouse was still ill, but alive on the fifth day: it was killed, and cultivations were made of the heart's blood and the spleen tissue, but no colonies of any bacteria made their appearance. I take it, then, that the two mice were made ill on account of having injected into them a large dose of the chemical products of the bacilli, the bacilli themselves not surviving or multiplying in the body of the mouse, and therefore the mouse must be also considered as refractory to the bacillus of fowl enteritis, altogether different from fowl cholera, to which illness mice are very susceptible. 


\section{CHAPTER XIII}

ATTENUATION OF THE VIRUS OF FOWL ENTERITIS

THE next question that I wished to answer was, whether it is possible to find a means of attenuating the virulence of cultures of the bacillus of fowl enteritis, in order to discover, if possible, a means of protective inoculation? For this purpose two preliminary questions had to be answered: (I) Do the bacilli form spores? (2) Does a mild attack of the disease protect the animal against a second fatal attack?

I. As regards the formation of spores. In all experiments of attenuating the virulence of a microbe, the presence or absence of spores is of first importance. The spores being possessed of a greater power of resistance would, therefore, evade the attenuating influence of adverse conditions, such as certain degrees of higher temperatures, chemical agencies, etc. The bacilli of a culture not having the power of forming spores might become attenuated in virulence, though of course not killed, by degrees of temperature such as $55^{\circ} \mathrm{C}$. and more, or by the addition of small doses of 
certain antiseptic media or other substances; whereas if spores are formed by the bacilli, the application of such conditions would be altogether productive of a different result.

Now the experiments of feeding which have been recorded in the preceding chapter point strongly to the absence of spores, because if such were present in the intestinal contents, or in the cultures used for the feeding, the positive results would have been more numerous than they have actually been. But these results do not absolutely demonstrate the incapability of the bacilli of forming spores, and for these reasons : under natural conditions the fowls, just as in fowl cholera, contract infection most probably by picking up food from a soil tainted with the evacuations of birds affected with the disease; and it might be possible that the bacilli on and in the soil produce spores, but they do not do so within the living body of the animal, or in the artificial cultures that had been used.

A careful microscopic examination of old cultures shows that there are present numerous bacilli which, from their swollen-up condition and their abnormal shape (spindle-shaped and abnormally thick), are no doubt comparable to involution forms. Besides there are present numerous bacilli which, from their not taking the stain, compare to dead bacilli, of which 
only the sheath is left. (Fig. 50 shows well such forms.) In old broth cultures, three and more weeks old, filamentous and cylindrical bacilli are met with numerously, which clearly show the granular degeneration and gradual disappearance of the protoplasm, so that then only the empty sheath is left. Such forms contain spherical or oval or irregular granules, which still take the dye; but the older the culture, and the more numerous these forms, the less likely is it found that the culture has retained vitality; that is to say, the less likely is it possible to start a new living sub-culture from them. I have made a large number of experiments, and have had broth cultures from several weeks to several months old, in which such forms (i.e. cylindrical or filamentous bacilli, containing from place to place, in an almost empty sheath, only here and there a larger or smaller stained particle) abounded, yet no living sub-culture could be started from them. These stained particles do not present any uniformity either in shape, size, or position, and there would be, therefore, no justification for regarding them as spores; besides, the death of old broth cultures containing those forms would clearly negative their being of the nature of spores. But also, by direct experiment, it is possible to show that neither in the bacilli in the fowl, nor in the cultures, is there anything present that is capable 
of resisting those tests generally considered indicative of the presence of spores. Simple but thorough drying in a thin film kills all bacilli; exposure to a temperature of $60^{\circ} \mathrm{C}$. for ten minutes sterilises the bacilli completely. Numerous experiments were made in this direction; the result was in all cases uniform. While heating to $60^{\circ} \mathrm{C}$. for five minutes was not reliable, heating to $60^{\circ} \mathrm{C}$. for ten minutes in all instances killed the bacilli.

From recent and old broth, gelatine, and Agar cultures numerous inoculations were made into gelatine and into alkaline broth in tubes; these were then exposed to $60^{\circ} \mathrm{C}$. for ten minutes, placed in the incubator, the first at $20^{\circ} \mathrm{C}$., the latter at $35-37^{\circ} \mathrm{C}$, but they all remained sterile. Broth cultures from twenty-four hours to three weeks old, and gelatine cultures from one to six weeks old, were tested for the vitality of the bacilli by establishing sub-cultures from them, then exposing the original cultures to $60^{\circ} \mathrm{C}$. for ten minutes. After this sub-cultures were again made. While the sub-cultures made from the original culture before heating proved successful, the sub-cultures made from the heated original culture remained sterile. It follows then from these experiments that there is no evidence that the bacillus of fowl enteritis is capable of forming spores.

2. Does one mild attack of fowl enteritis protect a 
fowl against a second attack? It must be evident that unless this is the case, that is, unless an animal becomes refractory to a second attack by having passed through a first mild attack, a primary inoculation with attenuated virus would be useless; it is for this reason imperative to ascertain whether, in a particular infectious disease, an animal having had the disease in a mild form becomes thereby possessed of immunity against a second attack. We have mentioned in a previous chapter experiments (Experiment 4 ) in which fowls had been the subject of inoculation with cultivations of the bacillus of fowl enteritis, and had become ill but had recovered. In addition to these fowls (three in number) five more fowls had recovered from the disease after inoculation with culture-these five animals will be mentioned below in connection with other series of experiments-so that there were eight fowls that had passed through an attack of the disease but had recovered. When they had seemingly quite recovered - they were lively, fed well, and appeared in all other respects well-they were subjected to a second inoculation, some with considerable doses of a recent first broth sub-culture, that is, a broth sub-culture made from a recent gelatine culture of the heart's blood of a fowl dead of the disease; others with blood or spleen tissue of a fowl dead of the disease. The same broth culture was used at the same time for in- 
oculation of two control fowls; both these latter became ill on the fifth day with the typical diarrhœa, they were quiet and off their food. No control experiments were made for testing the virulence of the blood and spleen tissue, because I considered this an unnecessary repetition; the experiments described in the previous chapter were so clear and decisive as to the virulent character of the blood and spleen tissue of a fowl that had died of fowl enteritis, that it did not require any further confirmation. The above eight fowls that were thus subjected to a second inoculation with virulent material showed no signs of any illness, they remained lively, fed well, and at no time showed any diarrhœa, and they remained well for weeks after, till they were discarded. It follows from these experiments that fowls that have passed through a first mild attack of the disease are possessed of immunity against a second attack. It was now most important to discover the material that could be relied upon as producing a distinct but transitory attack of fowl enteritis.

The first experiments were made with broth subcultures from a gelatine culture of the heart's blood of the rabbit mentioned in the previous chapter (sub. 8), as having been the only rabbit out of six that had died after inoculation with broth culture. Seeing that rabbits are so little susceptible to this disease, I thought it not improbable that the bacilli taken from the body 
of the rabbit might have lost some of their virulence, since the body of this animal is evidently not a favourable medium for their growth and multiplication. On testing by experiment this supposition, it did not prove correct. Twelve fowls were inoculated with broth sub-cultures of the bacillus derived from the rabbit. The result was this - that all twelve birds had diarrhœa on the $5^{\text {th }}$ or 6 th day, and that nine died (between the 7 th and 9 th day) and only three ${ }^{1}$ survived. A broth sub-culture derived from the rabbit proved, therefore, of a considerable degree of virulence, worse than useless for protective inoculation, since 75 per cent of the animals inoculated died.

Another set of experiments was this: a fowl had died of fowl enteritis, after inoculation with culture, on the roth day, that is later than usual, and I surmised that possibly the bacilli in this fowl might be of an attenuated virulence. From gelatine cultures of the heart's blood of this fowl broth sub-cultures were then made and after 24 hours' incubation at $37^{\circ} \mathrm{C}$. were used for the inoculation of twelve fowls. Seven of these died of typical fowl enteritis, the other five, ${ }^{2}$ though ill between the $5^{\text {th }}$ and 9 th day, survived.

1 These three fowls were mentioned above as having been afterwards used for a second inoculation with virulent material and proved refractory.

2 These five fowls were mentioned above as having been used for a second inoculation with virulent material and proved refractory. 
This broth culture proved, therefore, also useless for protective inoculation.

The next attempts were directed towards attenuating virulent broth cultures by heat: ( $\mathrm{I}$ ) by exposing broth cultures incubated at $37^{\circ} \mathrm{C}$. from 24 to 48 hours to a temperature of $50^{\circ} \mathrm{C}$. for 10 minutes. Of such a culture about $\frac{1}{4}$ of a cubic centimetre was injected subcutaneously into each of ten fowls. All these fowls became ill with fowl enteritis, eight died, two survived. These two were then re-inoculated with virulent broth culture, and proved refractory.

(2) Of broth culture exposed to $50^{\circ}$ C. for 20 minutes, about $\frac{1}{4}$ of a cc. was injected into each of eleven fowls. All became ill, four died, seven survived. These seven were also re-inoculated and proved refractory.

(3) Of broth culture exposed to $55^{\circ}$ C. for I 5 minutes about $\frac{1}{4}$ of a cc. was injected into each of eight fowls. All these fowls were quiet on the 6 th, 7 th, and 8 th days, were off their food, but had no diarrhœa ; seven survived and became well again, ${ }^{1}$ but the eighth was found dead on the 8th day with enlarged spleen; cultivations made of the heart's blood proved that the animal had died of fowl enteritis. This series seemed, therefore, a considerable step in advance of the former.

1 Also these seven fowls were re-inoculated with virulent broth culture, but proved refractory. 
(4) In this series the broth culture was heated to $55^{\circ} \mathrm{C}$. for 20 minutes and then inoculated in the same quantity as before into eight fowls. They were all quiet and off their food on the 6th and 7 th days, but there was no diarrhœa; on the Ioth day they seemed to all appearances right again. This was then the result aimed at, and it now only remained to be proved that these same fowls were capable of withstanding a second inoculation with virulent material. For this purpose a broth culture, 24-48 hours incubated at $37^{\circ} \mathrm{C}$., was used for inoculation, two control fowls being at the same time inoculated with the same broth; each of these ten fowls (that is the eight that had been subjects of the first inoculation and the two control fowls) received one whole Pravaz syringeful, i.e. a considerable dose. The two control fowls became ill and were dead on the 7 th day from the typical fowl enteritis, the eight other fowls remained perfectly well. From this, I think it follows that a recent broth culture heated to $55^{\circ} \mathrm{C}$. for 20 minutes can be taken to represent a fluid suited for protective inoculation. Such a broth culture when tested by sub-culture yields good and virulent growths, so that while the heating to $55^{\circ} \mathrm{C}$. for 20 minutes impairs the virulence, it does not impair the vitality of the bacilli.

(5) A series of experiments which deserves mention was instituted to see, whether uncler natural conditions 
infection is carried from animal to animal by air, or whether, as we mentioned in a former chapter as very probable, infection is produced by ingestion-the animals picking up food from soil tainted with the evacuations of a diseased fowl. Mr. W. Cook, of Orpington, kindly placed at my disposal a plot of ground, not situated at or near his poultry-farm. This plot was divided by wire netting into two adjacent sections; the wire netting separating these was made double, and a space or passage of about is inches was left free between them. Into each section were placed ten healthy fowls, which we will call lot $\mathrm{A}$ and lot $\mathrm{B}$ respectively. All fowls of lot $\mathrm{A}$ were then inoculated with broth sub-culture of the bacillus of fowl enteritis, the fowls of lot $B$ remaining untouched. All of lot A were distinctly ill on the fifth day; they were quiet and had diarrhœea between the fifth and eighth day ; seven died, three survived. ${ }^{1}$ While the disease was rife amongst lot $A$, one fowl escaped from lot $B$ and found its entrance amongst lot $A .{ }^{2}$ After a few days sojourning amongst them it became also ill and died of the fowl enteritis; also a strange fowl not belonging to either of the two lots got access to lot $\mathrm{A}$ :

I After a fortnight, when they seemed quite well again, they were re-inoculated, but proved refractory.

2 The two plots of ground had a covering of wire netting, but this was not perfect. 
this too became ill with the fowl enteritis and died. But the nine fowls of lot $B$ remained well many days after the disease had come to an end in lot A. So that though a wire netting separation with only i 8 inches interval divided the fowls of lot A from those of lot $\mathrm{B}$, the disease from lot $\mathrm{A}$ was not communicated to the latter fowls; but on the other hand, the two accessory fowls that had lived with the diseased fowls of lot $\mathrm{A}$ in the same enclosure became infected.

The nine control fowls of lot B were afterwards inoculated with broth cultures, they all became ill on the fifth day, and suffered from diarrhœa; eight died, one survived.

From what has been stated in the preceding two chapters, it is firmly established, I think, that both in the nature and course of the disease, and in the character and distribution of the bacilli, and in the susceptibility of some and non-susceptibility of certain other animals, fowl enteritis and fowl cholera are two well-differentiated diseases. As has been shown, particularly in the last chapter, the uniformity in the symptoms and the duration of the incubation period of the disease observed on a very large number of fowls-nearly five dozen fowls were experimented upon in the last five series of experiments-prove conclusively the difference between the two diseases. But I have some further experiments to record that 
confirm this in a striking manner, though they were undertaken with a different object. While investigating the action on animals of different pathogenic microbes, when introduced simultaneously or separately into the same animal, I tested also in this direction the action of the bacillus of fowl cholera and fowl enteritis. Pigeons, as we mentioned on a former page, are very susceptible to fowl cholera, but quite refractory to fowl enteritis. I inoculated $(a)$ two pigeons with fowl enteritis culture (broth culture 48 hours old), (b) two pigeons with fowl cholera cultivation (broth culture 48 hours old), and (c) two pigeons with a mixture in about equal parts of the same two broth cultures.

The pigeons a remained well and alive, the two pigeons $b$ and the two pigeons $c$ were found dead after 48 hours, from typical fowl cholera. The two pigeons $a$ were then after about ten days inoculated with fowl cholera cultivation (broth culture $4 S$ hours old); both were found dead before 30 hours were over from typical fowl cholera.

One fowl and one pigeon were inoculated with a broth culture of the bacillus of fowl enteritis. The fowl became ill with the typical fowl enteritis, but the pigeon remained, as a matter of course, unaffected. The fowl, though at first very ill, nevertheless clid not die, but recovered gradually. After the lapse of I4 
days the fowl seemed perfectly well again; it and the pigeon were then inoculated with broth culture of the bacillus of fowl cholera. At the same time one control fowl and one control pigeon were inoculated with the same culture. Both the pigeons were dead in I 8 hours of typical fowl cholera; both fowls were very ill during the second, third, and fourth days, very drowsy, had copious diarrhœea, but both gradually recovered.

From these experiments it follows, (I) that while both the pigeon and fowl are susceptible to fowl cholera, the pigeon in a higher degree than the fowl, this is totally different as regards fowl enteritis, since the pigeon is refractory and the fowl very susceptible; (2) that a first attack from fowl enteritis does not protect the fowl from an attack of fowl cholera.

Although the two cliseases are different, the mode of their spread and the general behaviour of the two species of bacilli are similar, and therefore the rules that should guide us in the prevention of the spread of either should be the same. These may be summarised in saying-(I) every fowl that shows any suspicion of the disease should be at once removed, killed, and burned; (2) the remaining fowls should be at once transferred to new ground, and, if practicable, 
should be subdivided in separate small lots; (3) the ground from which the affected fowls have been removed should be turned, disinfected with quicklime, and not used for fowls for a considerable time. These seem to me the best and easiest ways to prevent the healthy fowls contracting the infection by picking up food tainted with the evacuations (full of the specific bacilli) of the diseased fowls. 


\section{CHAPTER XIV \\ "CRAMPS" IN YOUNG PHEASANTS}

During my stay (June $\mathbf{1} 887$ ) at Blairquhan, Mr. Douglas, the head gamekeeper, then in the employ of G. Bailey Worthington, Esq., called my attention to a great mortality from "Cramps" existing amongst the young pheasants then reared by him. $\mathrm{He}$ was in great distress on account of the helplessness in which he found himself in the matter. The utmost care, the most scrupulous attention to all details necessary in the rearing of pheasants exercised by him and those serving under him, availed nothing to neutralise or stem the progress of the disease; daily new birds showed signs of the disease, and daily, when he in the morning went round the grounds and inspected the coops, several new deaths had occurred amongst the young birds. From what I have observed myself, the first signs of illness manifest themselves in lameness of one leg and in the unwillingness of the birds to move; next day both legs are lame, the birds are sitting quietly, and when made to move are seen to 
drag, more or less, their feet along the ground ; generally next day, or the day following, this condition becomes more intensified, the birds do not move at all, their eyes are closed, they do not of course feed any more, and soon after the animals are found dead. This disease is known to keepers and pheasantbreeders as the "cramps," but I think it would be more appropriate to call it paralysis, which would be more in harmony with the inability to move, though in reality it is neither the one nor the other. During the two days that I stayed at Blairquhan I saw a considerable number of animals thus affected or dead from the disease, and I had the opportunity of making a post-mortem examination on a good number. Afterwards Mr. Douglas forwarded to me in London a considerable number of young pheasants that had died of the disease, and these also were carefully examined. All the birds were a few days to a few weeks old, the disease occurring most commonly during the second to third week; and all without exception showed, as the only constant and conspicuous feature, a disease of the bones of the extremities - always of the legs, in some few instances also of the bones at the anterior extremities. The disease may be compared to an acute periostitis and osteomyelitis. The femur of both legs, and the tibia very often, showed either at the upper or the lower end of the shaft, or at 
both, a localised congested state of the periosteum, often hæmorrhage into this and into the surrounding muscle; the bone itself is thinned at these places or completely dissociated either in a circular line comprising the whole circumference, or obliquely, so much so that between the actual condition and what one would consider a fracture-simple fracture in the former, oblique or compound fracture in the latterthere seemed a complete analogy. The first few birds dead of the disease that I dissected at Blairquhan showed the fracture at the upper or lower end of the shaft of the femur and tibia, particularly the former, so pronounced that Mr. Douglas himself in the next few birds could easily discover this condition. Hæmorrhage into the periosteum and the surrounding muscle occurs oftener in the upper and lower ends of the shaft of the femur than in the tibia, and chiefly in connection with oblique fracture. I thought at first that the condition of the bones might have been produced accidentally, either by the foster-mother or by other animals attacking the little pheasants. But Mr. Douglas assured me that this is quite out of the question, and, in fact, impossible that such a thing should happen unknown to him and his assistants, the rearing being carefully watched night and day. The large number of birds that died from the same disease during June of that year, day after day, and that had 
died in previous years, notwithstanding the utmost care and attention, made it, therefore, clear that we had to deal with an epidemic disease. The principal symptom of the disease, viz. the impaired movement observed by the keepers in many parts of the country, south and north, and the cause of its appellation, viz. "cramps," made it also clear that the disease is connected with disease of the limbs, and it is hardly credible that this should everywhere be the same, and the result of an accident that had everywhere escaped the watchfulness of the attendants. But what did surprise me is the fact that both in the epidemic in Scotland, which I then observed, as well as in young pheasants sent to me through the Field from the south of England that had also died of the "cramps," the remarkable condition of the bones-femur and tibia - should have entirely escaped notice. And, moreover, a correspondent of the Field thought it "absurd" my saying that the young pheasants dying of the "cramps" have this condition of the bones, viz. partial and complete dissociation of the ends of the shaft. I can only repeat that in no single instance of young pheasants dying of the "cramps" have I missed this condition, but I must add that I know of an epidemic disease among young pheasants leading to death in which this condition is not found, and which animals do not die of the "cramps," but are 
affected with a localised necrotic disease of the mouth, throat, eyelids, and skin of the lips, contracted from their foster-mothers, the hens, affected with a wellknown chronic localised necrotic thickening of the mucous membrane of the tongue and throat. The two diseases, "cramps" and necrotic stomatitis and dermatitis in young pheasants, are in symptoms and anatomical changes utterly distinct diseases.

As mentioned above, the principal anatomical change in "cramps" of young pheasants is found in the periosteum and the bone near the ends of the shaft of the femur and tibia, occasionally but rarely also of the humerus.

On making sections through the affected parts of the bone and its periosteum, the changes are those which constitute periostitis and osteomyelitis. The vessels of the periosteum at the ends of the shaft are engorged with blood, extravasated blood being noticed in the tissue of the deep layer of the periosteum; this, at the same time, is thickened and crowded with round-cells or inflammatory corpuscles. The inflammation or infiltration with round-cells or leucocytes extends into the bone, all Haversian spaces and Haversian canals being densely infiltrated with them, the bone matrix either totally or partially destroyed, absorbed; the infiltration with round-cells of the bone extends into, and 
forms a continuity with, the inflamed central marrow, this showing its blood-vessels much distended by blood and its tissue a continuous mass of round-cells. From the places of greatest inflammation, i.e. at the ends of the shaft, the engorgement of the vessels and the infiltration with leucocytes of the bone and the central marrow extend a little way beyond. This condition of periostitis and osteomyelitis is present in all the cases which I examined, and the rarefaction and absorption of the bone at the end of the shaft and the subsequent dissociation is hereby easily explained.

In cover-glass specimens made of the inflamed tissues, and in sections through the inflamed parts, a few minute oval or cylindrical bacilli are constantly met with (they require for demonstration prolonged staining of the sections - a couple of hours and more in methyl blue aniline water; or staining for half an hour to an hour in gentian violet aniline water shows the bacilli well). Of course it is necessary after the staining to well decolorise the section in methylated spirit in order to remove as much as possible the dye from the tissue cells, the bacilli retaining the dye more persistently. From several cases cultivations were made; with sterile scissors the bone is laid bare, with another pair of sterile scissors an incision is made into the inflamed part, then with a sterilised platinum loop a small particle of the marrow is removed and trans- 
ferred to test-tubes containing nutrient gelatine and nutrient Agar set with slanting surface, and is well rubbed over the slanting surface. The gelatine tubes are incubated at $20^{\circ} \mathrm{C}$, the Agar tubes at $36-37^{\circ} \mathrm{C}$. All the tubes thus inoculated developed several colonies of one and the same species of bacilli. In the gelatine tube I noticed after 2-3 days the first indications of the colonies in the shape of minute translucent greyish droplets; in Agar the colonies are noticeable after one day's incubation. The colonies, even when well developed, are from the size of a pin's point to that of a millet seed. Streak subcultures on gelatine are very characteristic, this being due to two facts- $(a)$ the slowness of the growth, the line of inoculation becoming marked as a line of small greyish transparent droplets, not before two to three days; $(b)$ as the droplets enlarge they have a tendency to become more or less confluent, but they always remain recognisable as individuals-there is not a continuous band-like growth produced, as in most streak cultures of bacilli. The same applies to Agar streak cultures, only of course the growth is faster, but also here the individuality of the colonies remains more or less preserved even where the colonies are situated closely and uninterruptedly side by side. In old gelatine- and in old Agar cultures the colonies become whitish, raised, knob-like, their outline slightly 
crenate. In stab cultures the line of inoculation becomes marked as a grey line made up of separate droplets. The gelatine is never liquefied by the growth.

In fresh specimens made of the colonies, i.e. examining a particle of the growth in sterilised salt solution or in broth, the bacilli are without motility; in such specimens, as also in cover-glass specimens dried and stained, the microbes are short bacilli rounded at their ends, many are short ovals, some cylindrical; there are always some cylindrical and rod-like bacilli present which are thicker in the middle portion than at the ends, they look, therefore, spindleshaped; they occur singly or often as dumb-bells. In specimens made of gelatine cultures the bacilli differ somewhat from those grown on Agar, inasmuch as in the former most of the microbes are short ovals, and in stained specimens show at each end in the transparent sheath a stained granule. These look very much like the typical fowl cholera bacilli; but also in specimens of gelatine culture there are some which are rod-shaped or even distinctly cylindrical; but not so numerous by any means as in Agar cultures. In these latter the majority are rod-shaped or cylindrical, and amongst them occur such as are characteristically spindle-shaped; but also in Agar cultures there occur a good many that are short ovals. Figs. 52 and 53 
show well the differences between the bacilli in gelatine and Agar cultures respectively.

Measurements made in dried and stained coverglass specimens give the following numbers: thickness about 0.3 micro-millimetres; there is no difference in this respect between the bacilli grown on gelatine and those on Agar; the cylindrical spindleshaped bacilli are thickest and measure as much as 0.4 to $0.5 \mu$ in the thickest portion; the length of the majority of the bacilli from gelatine cultures is $0.4 \mu$ to 0.6, a few $0.8 \mu$, very few more than this- $1.6 \mu$. In Agar cultures the majority are 0.6 to 0.8 to i.o $\mu$ long, some up to $1.6 \mu$. The cultures and sub-cultures which I prepared from the young pheasants in June and July I 887 , and which I described in the Field, July 30 , I 887 , I put away ${ }^{1}$ during the following vacation, August and September, in order to resume the inquiry on my return towards the latter half of September, but unfortunately, to my great discomfiture, they were all found dead; every tube put aside, though it remained free of all accidental growth and looked in all respects preserved, was tested by sub-cultures, but with no result; none could be started from them, and therefore all further experimentation with them came to an end. I have not been able hitherto to procure

1 A few experiments on guinea-pigs and mice by subcutaneous inoculation of culture did not yield any definite result. 
new material, i.c. young pheasants affected with the "cramps," and am, therefore, unable to say anything further on this subject. But of this I am clear, that the disease is in pathological respects a well-defined, acute periostitis and osteomyelitis, and that it is associated with a definite and well-characterised species of bacilli, present in the inflamed bone, and obtainable in all cases by cultivation. 


\section{EXPLANATION OF ILLUSTRATIONS}

All Figures are reproductions from Photograms, except Figures I 5 and 16 , which are from drawings by Mr. M. H. Lapidge.

Figures 10, 11, 14, 1 7, 18, 19, 22, 32, 33, 34, 36, 41, 42, 43, 44, 48, and 50, are by Mr. Andrew Pringle, the remainder by Mr. E. C. Bousfield. 
Section through the Lung of Grouse dead of the Grouse D) isease. Magnifying power about 10.

The air-cells appear solid, being filled with blood and exudation. Two large air-spaces are shown, filled with homogencous exudation.

FIG. 2.

Section through the Liver of Grouse dead of the Grouse Disease. Magnifying power 1000.

a. Part of a large blood-vessel filled with blood-corpuscles.

b. Capillary blood-vessel plugged with the bacilli.

c. Liver cells.

FIG. 3.

A similar preparation as in Fig. 2. Magnifying power 1000 .

Several capillary vessels are shown, plugged with the bacilli.

FIG. 4.

Section through the Lung, showing a mass of capillary bloodvessels plugged with the Bacilli. Magnifying power $65^{\circ}$.

FIG. 5.

Plate cultivation of the Bacillus of Grouse Disease. Natural size.

Each of the small dots is a colony growing in the depth. Each of the larger white patches is a colony on the surface of the gelatine.

FIG. 6.

Streak-culture on gelatine of the Bacillus of Grouse Disease. Natural size. 


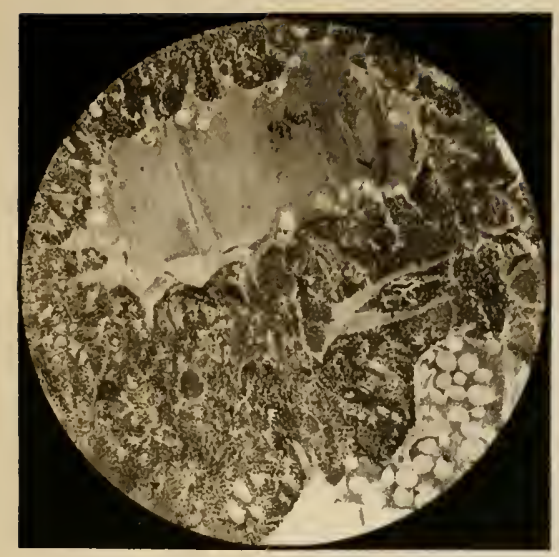

Fig. 1

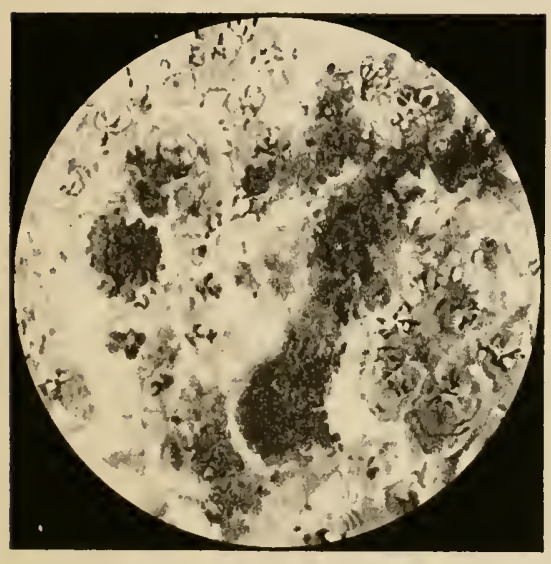

Fig. 3

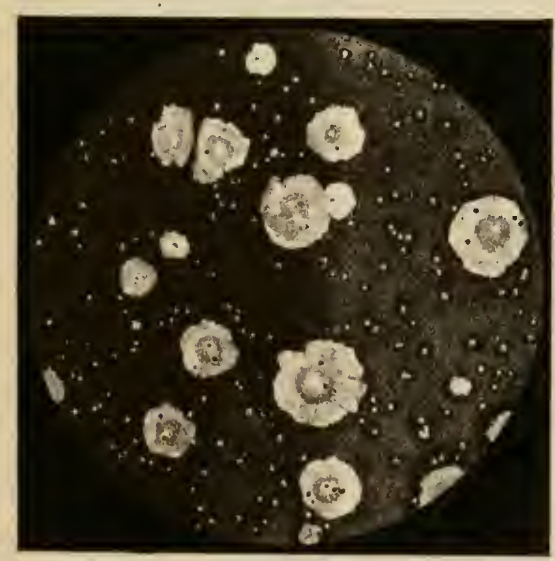

Fig. 5

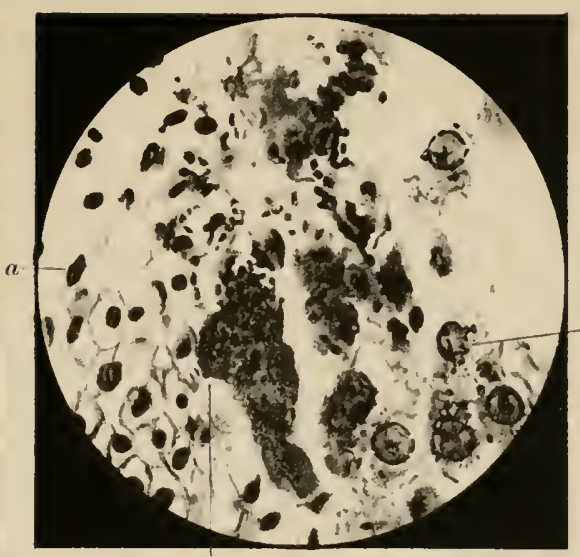

Fig. 2

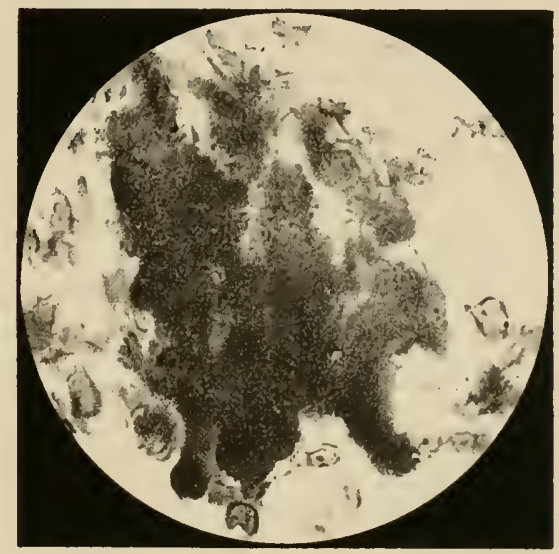

Fig. 4

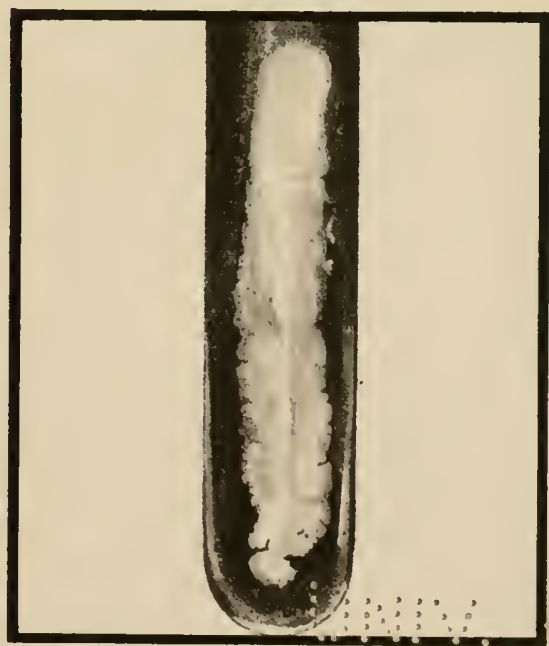

Fig. 6 , 




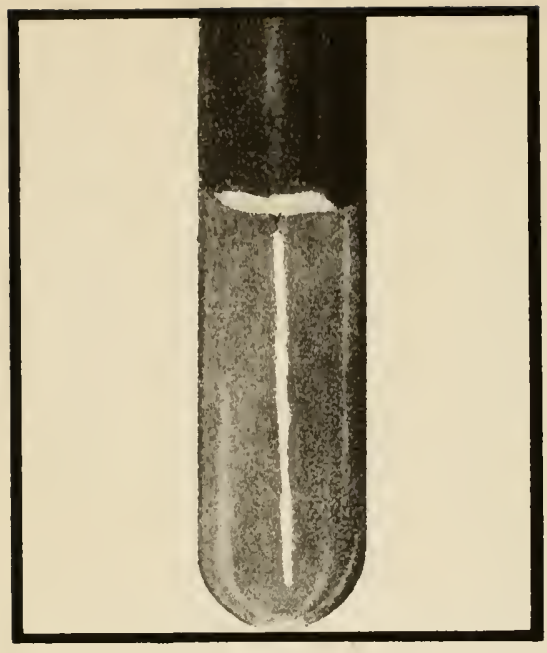

Fig. 7

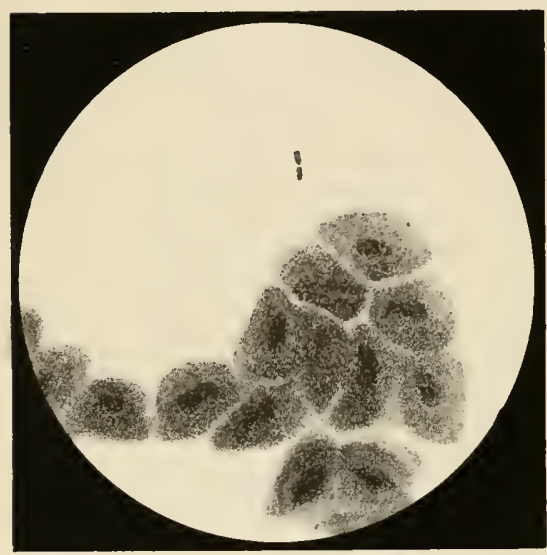

Fig. 9

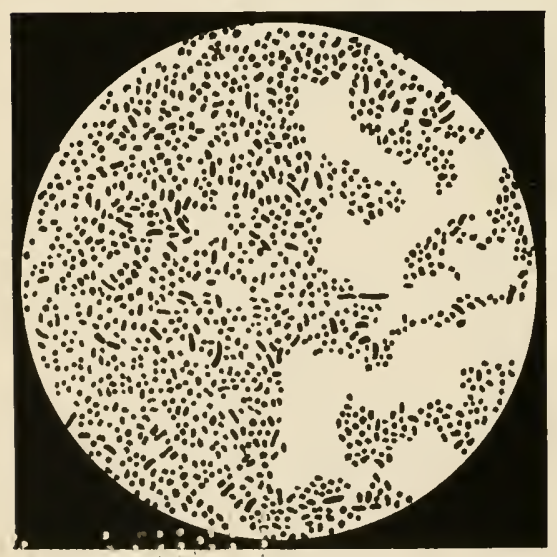

Fig, 11

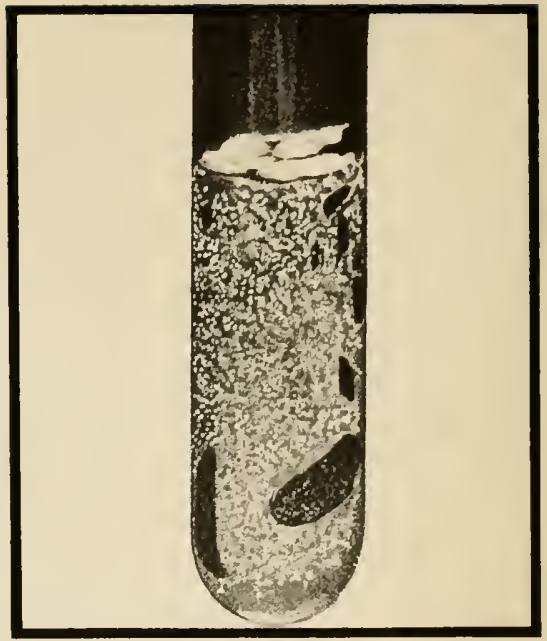

Fig. 8

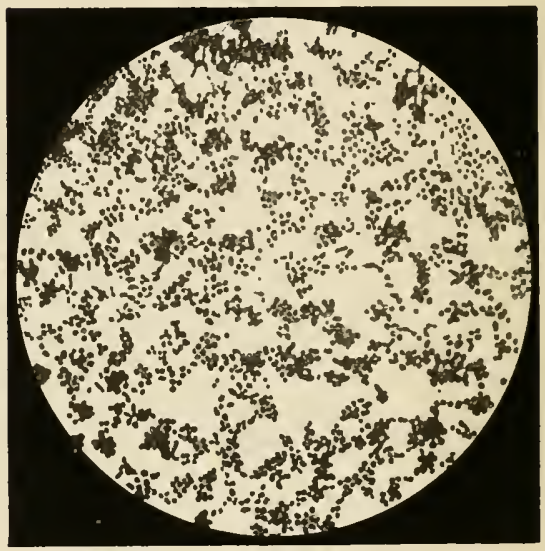

Fig. 10

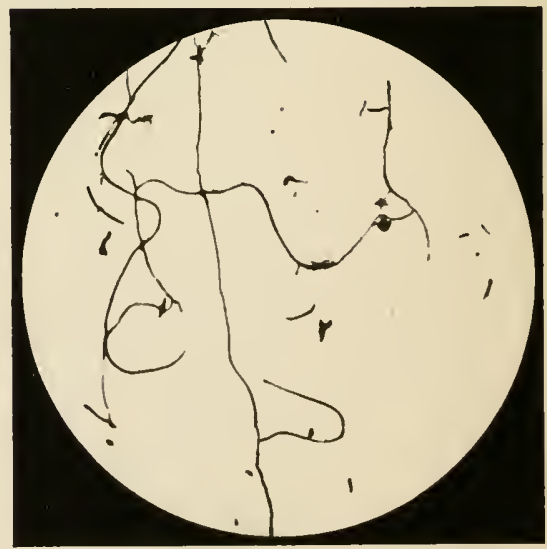

Fig. 12 


\section{FIG. 7 .}

Stab-culture in gelatine of the Bacillus of Grouse Disease. Natural size.

FIG. 8.

Shake-culture in gelatine, showing gas bubbles in the depth of the gelatine. Natural size.

\section{FiG. 9.}

Cover-glass specimen of the Lung Juice of Grouse dead of the Grouse Disease. Magnifying power 1000.

One dumb-bell of the bacilli is shown and several blood discs.

FIG. IO.

Cover-glass specimen from a Culture in gelatine of the Bacillus of the Grouse Disease. Magnifying power 1000.

FIG. II.

Cover-glass specimen from a Culture in gelatine of the Bacillus derived from the blood of an inoculated Mouse. Magnifying power 1000 .

FIG. 12.

Cover-glass specimen from a "Salted" Culture of the Bacillus of Grouse Disease (see p. 31). Magnifying power 1000. 


$$
\text { FiG. } 13 .
$$

Cover-glass specimen of the Lung Juice of an infected Mouse. Magnifying power 1000.

The bacilli are present in large numbers, singly and in masses.

FIG. I 4 .

A similar preparation as in Fig. I 3. Magnifying power 1000.

A swollen leucocyte filled with the bacilli.

FiG. 15.

From a section through the Liver of Grouse dead of the Grouse Disease, showing capillary blood-vessels filled with the Bacilli. Low power.

$$
\text { FIG. I6. }
$$

From a similar preparation as Fig. I5, but more highly magnified.

FIG. I 7 .

Cover-glass specimen of the blood of an infected Guinea-pig. Magnifying power rooo.

Numerous bacilli are seen, free and enclosed in leucocytes, the latter much swollen and disintegrating.

Fig. I 8 .

Cover-glass specimen of the blood of an infected Ammer. Magnifying power 1000.

Amongst the blood-corpuscles are very numerously the bacilli. 
Fig. 13
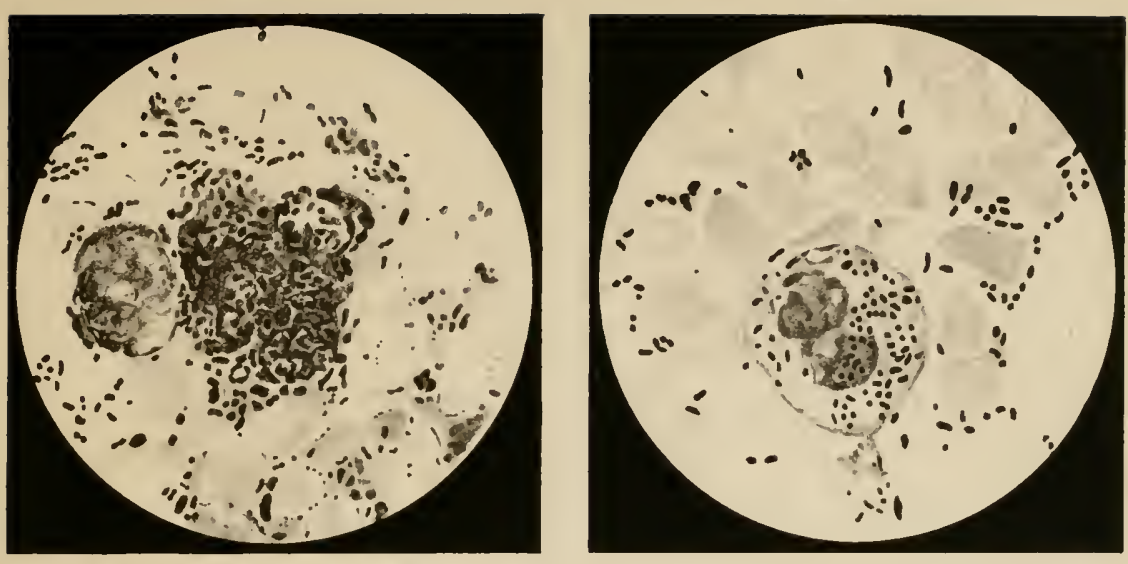

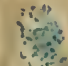

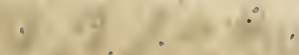

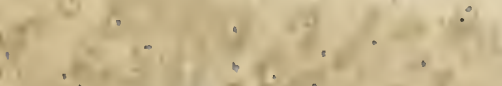
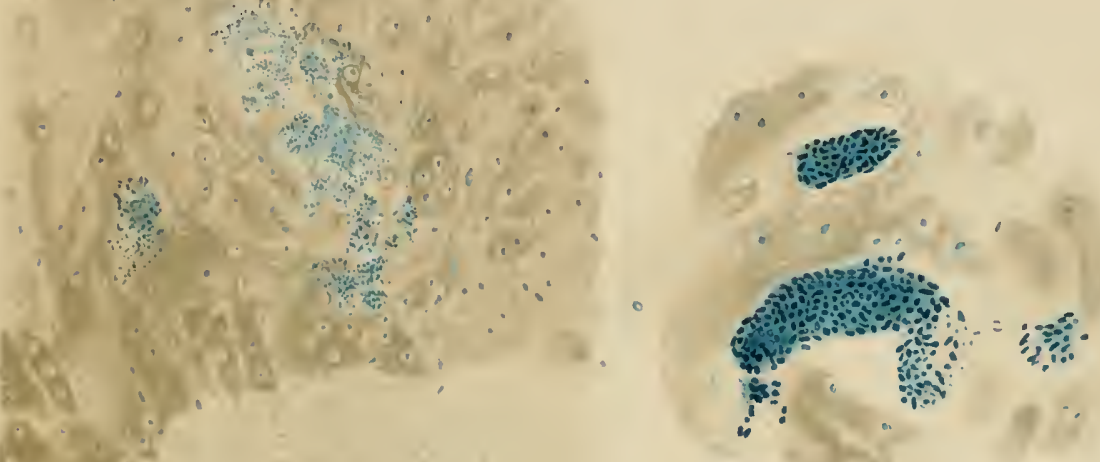

Fig. 16
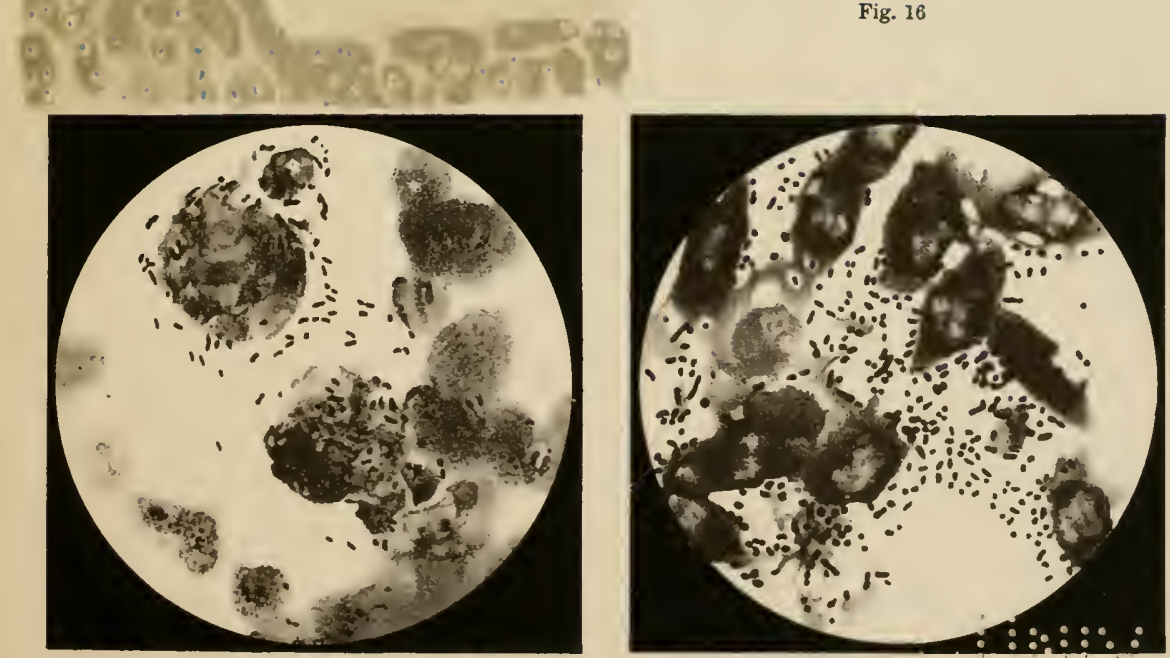

Fig. 17

Fig. 18 




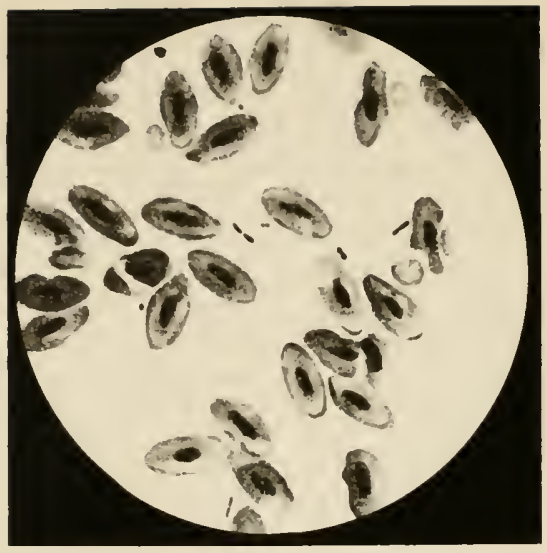

Fig. 19

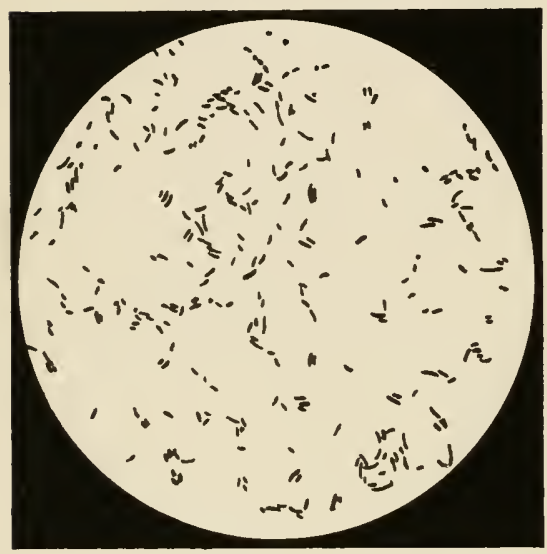

Fig. 21

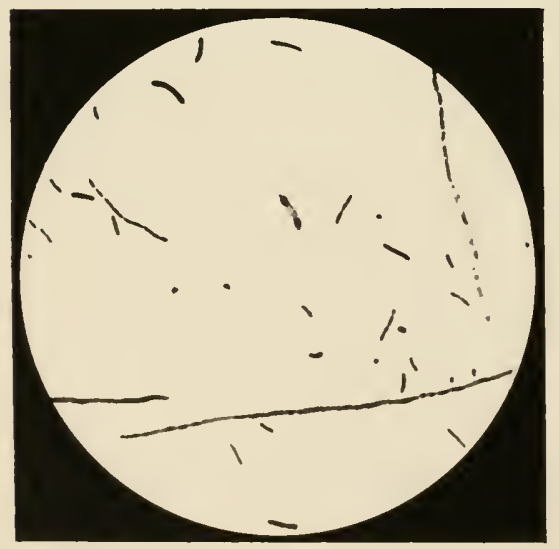

Fig. 23

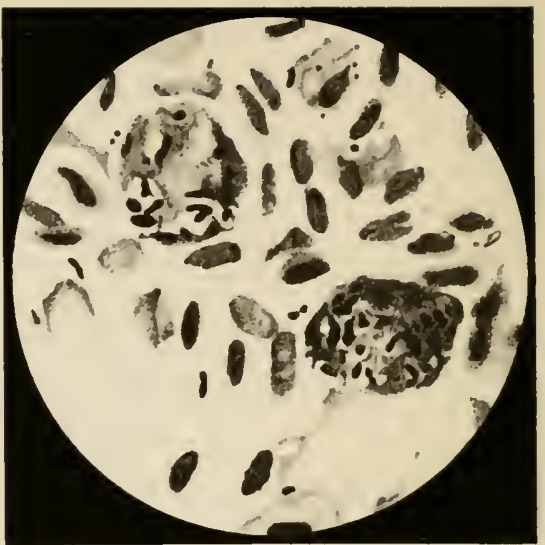

Fig. 20

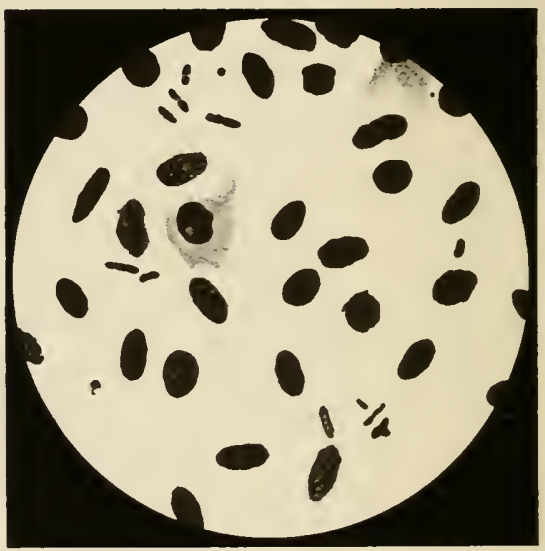

Fig. 22

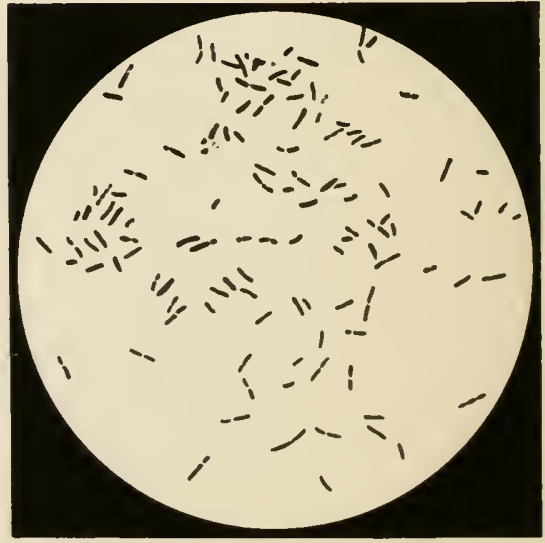

Fig. 24 
FIG. 19.

Cover-glass specimen of the Lung Juice of an infected Ammer. Magnifying power 1000.

Numerous bacilli are seen amongst the blood-corpuscles.

FIG. 20.

Cover-glass specimen of the Lung Juice of an infected Ammer. Magnifying power 1000.

Amongst the blood discs (of which only the nucleus is shown) there are isolated bacilli. Two leucocytes include in their substance the bacilli.

F19, 2 I.

Cover-glass specimen from a cultivation of the Bacilli derived from the blood of an infected Ammer. Magnifying power 1000.

FIG. 22.

Cover-glass specimen of the Heart's Blood of a Grouse dead of the Autumnal Grouse Disease. Magnifying power 1000.

Amongst the blood discs (of which only the nucleus is shown) are seen the bacilli.

Fig. 23 .

Cover-glass specimen of the Subcutaneous Edema Fluid of a Guinea-pig infected with the Erobic CEdema Bacilli. Magnifying power 1000 .

F1G. 24.

Similar preparation as in Fig. 23. Magnifying power 1000. 
FIG. 25 .

Cover-glass specimen from an Agar culture of the Arobic Edema Bacillus. Magnifying power $6_{50}$.

FIG. 26.

Similar specimen as in Fig. 25, but from a gelatine culture. Magnifying power 1000.

\section{FIG. 27.}

Leucocytes of the Subcutaneous Edema Fluid of an infected Guinea-pig; the cells contain the Bacilli in their interior. Magnifying power 1000 .

\section{FIG. 28.}

Plate culture of the Erobic Edema Bacillus. The small points are colonies in the depth; the large white patches are colonies on the surface of the gelatine. Natural size.

\section{Fig. 29.}

Streak culture on gelatine of the Ærobic Edema Bacilli. Natural size.

FIG. 30.

Stab-culture of same. Natural size. 


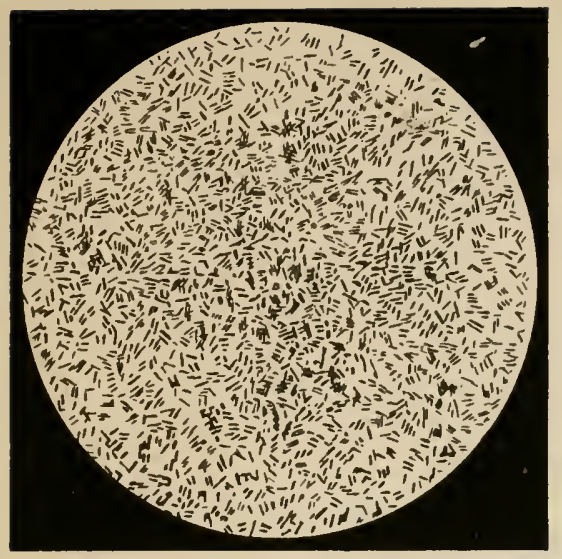

Fig. 25

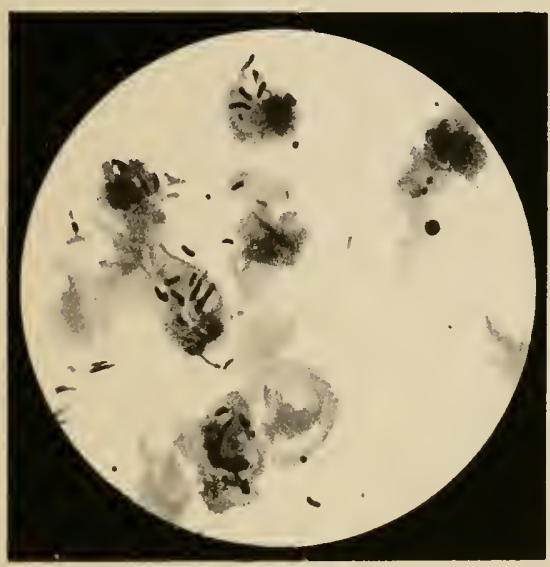

Fig. 27

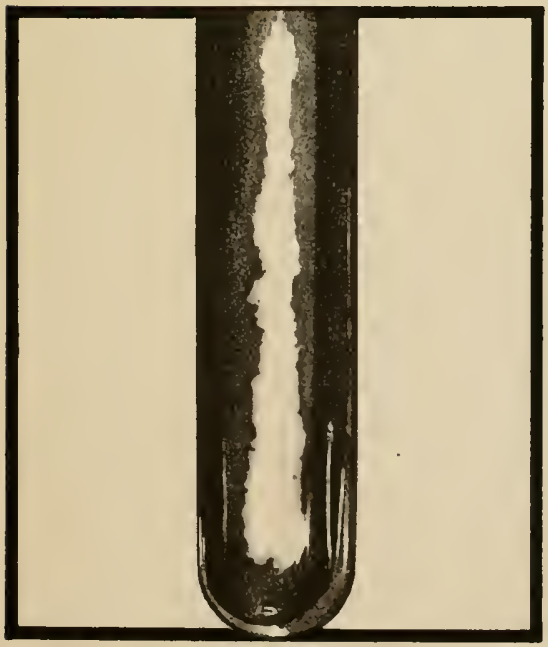

Fig. 29

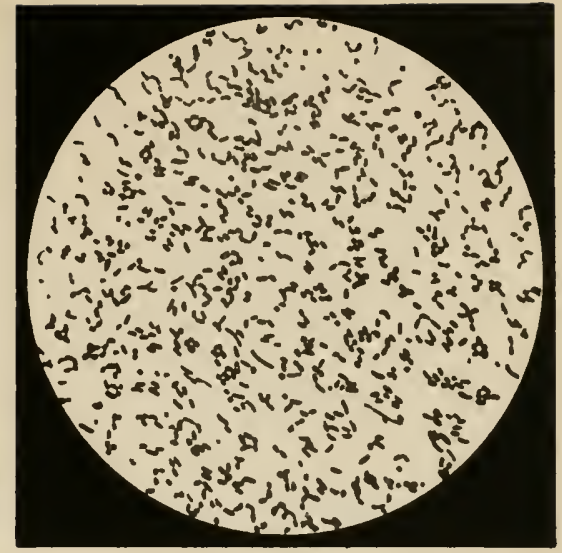

Fig. 26

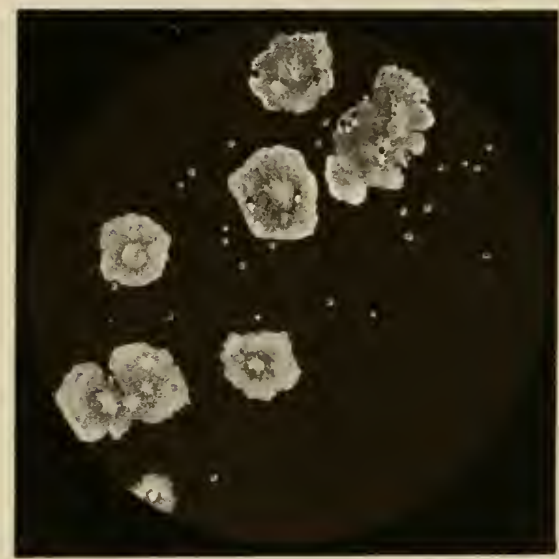

Fig. 28

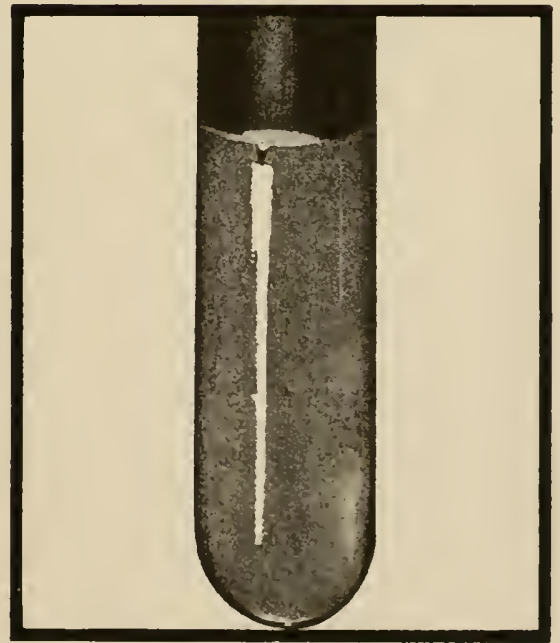

Fig. $3 C$ 



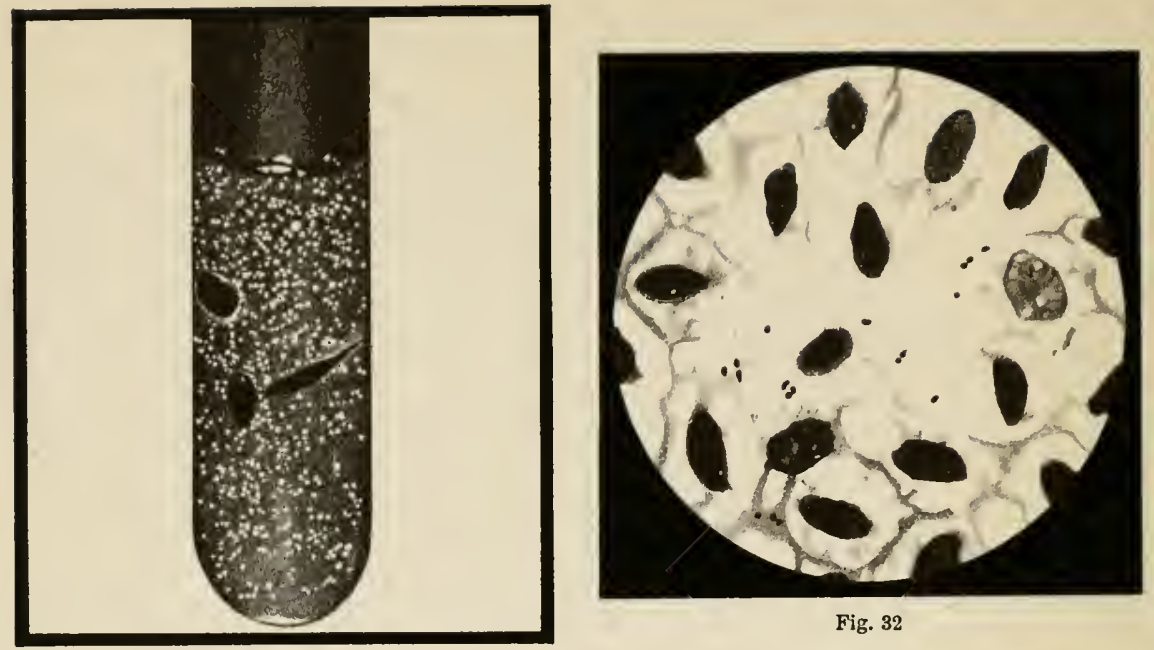

Fig. 31
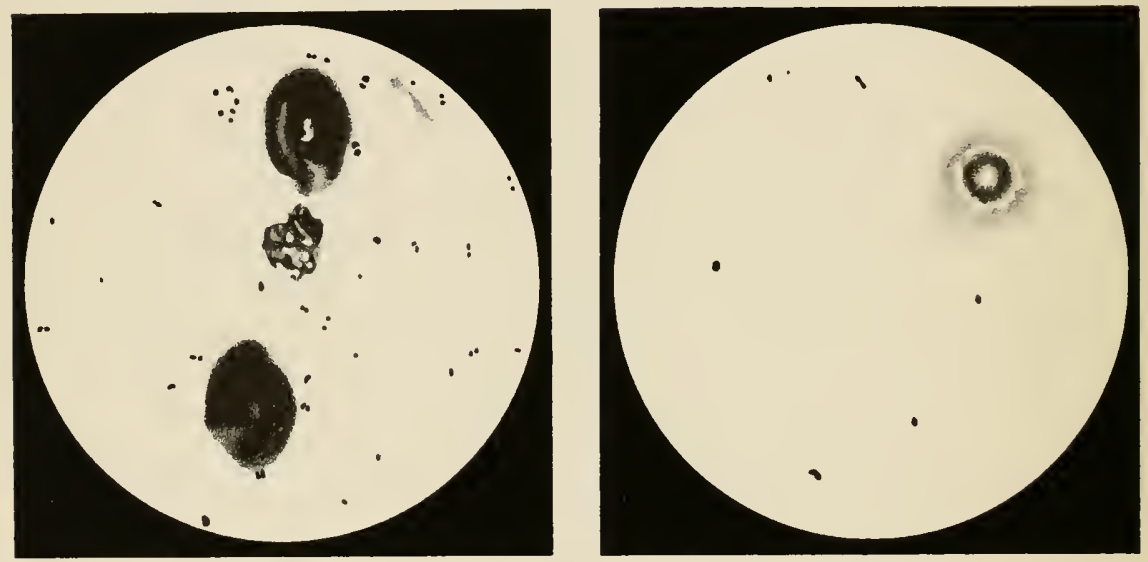

Fig. 33

Fig. 34
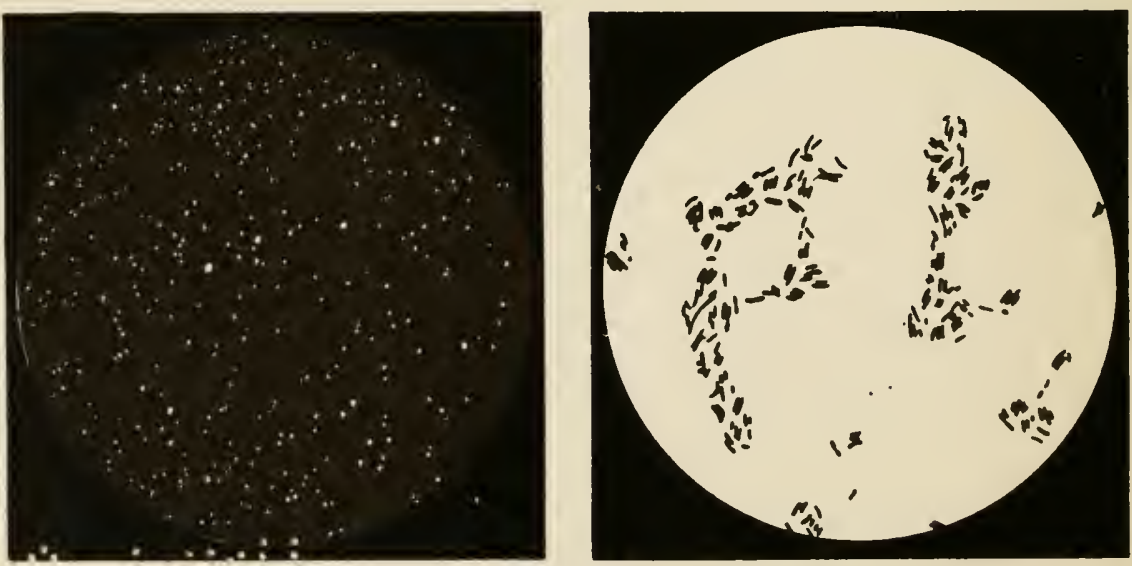


\section{FIG. 31 .}

Shake-culture of same, showing a few gas bubbles in the deeper parts of the gelatine. Natural size.

FiG. 32.

Blood of Fowl dead of Fowl Cholera. Numerous Bacilli of Fowl Cholera amongst the blood-corpuscles. Magnifying power I 000 .

FIG. 33.

Blood of Pigeon dead of Fowl Cholera. Magnifying power 1000.

FIG. 34.

Blood of Rabbit dead of Fowl Cholera. Magnifying power 1000.

FIG. 35 .

Plate culture in gelatine. Natural size.

The colonies are all extremely minute dots.

FiG. 36.

Cover-glass specimen (impression specimen) of a colony of the Fowl Cholera Bacillus. Magnifying power 1000. 
Fig. 37 .

Section through the Liver of a Fowl dead of Fowl Cholera. Magnifying power 1000 .

Many blood-vessels contain the bacilli very numerously.

FIG. 38 .

Section through the Liver of a Rabbit dead of Fowl Cholera. Magnifying power 1000.

FIG. 39 .

Streak culture on gelatine of the Fowl Cholera Bacilli. Natural size.

FIG. 40.

Stab-culture of same. Natural size.

FIG. $4 \mathrm{r}$.

Blood of Fowl dead of Fowl Enteritis. Magnifying power 1000. Very few bacilli amongst the blood-corpuscles.

FIG. 42 .

Cover-glass specimen of Spleen Juice of a Fowl dead of Fowl Enteritis. Magnifying power rooo.

Several bacilli amongst the tissue cells. 


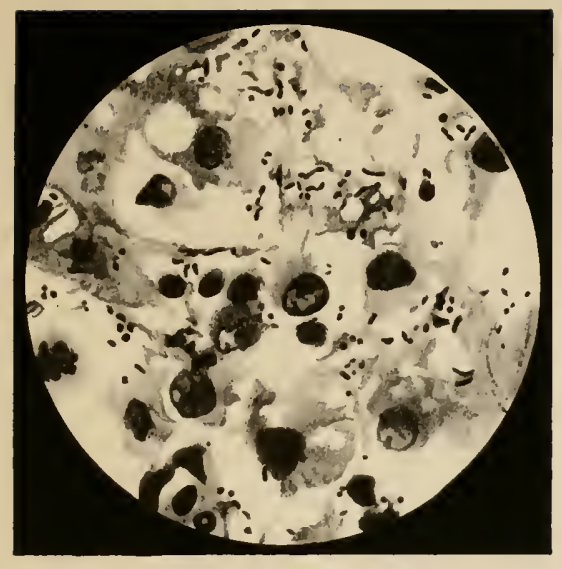

Fig. 37

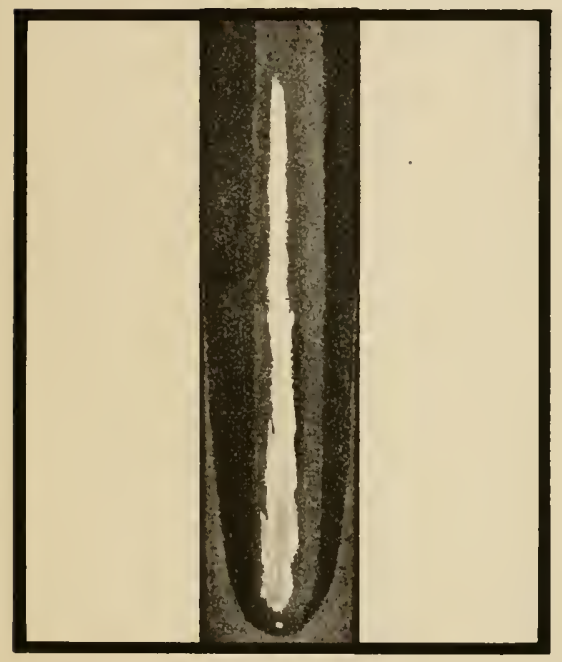

Fig. 39

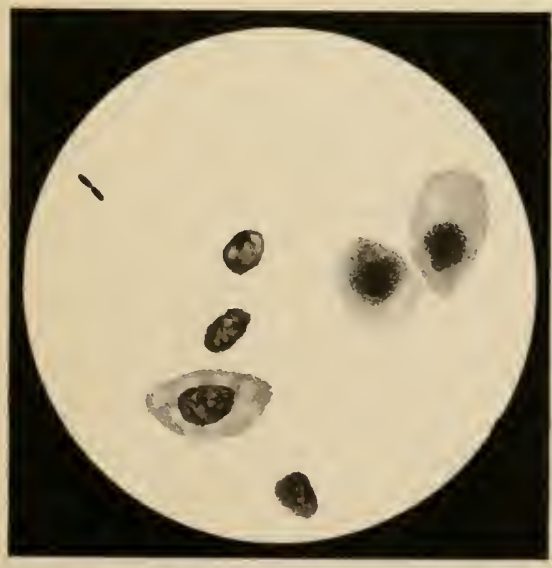

Fig. 41

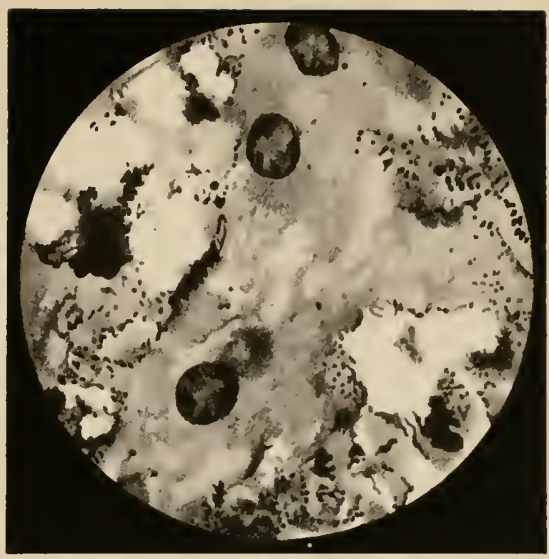

Fig. 38

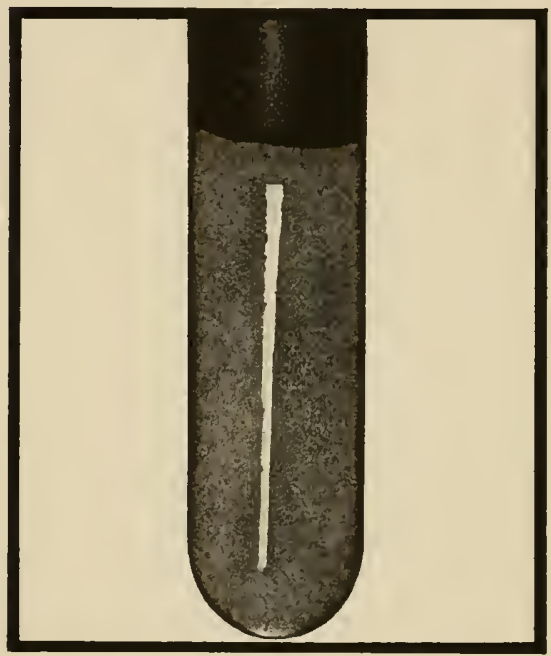

Fig. 40

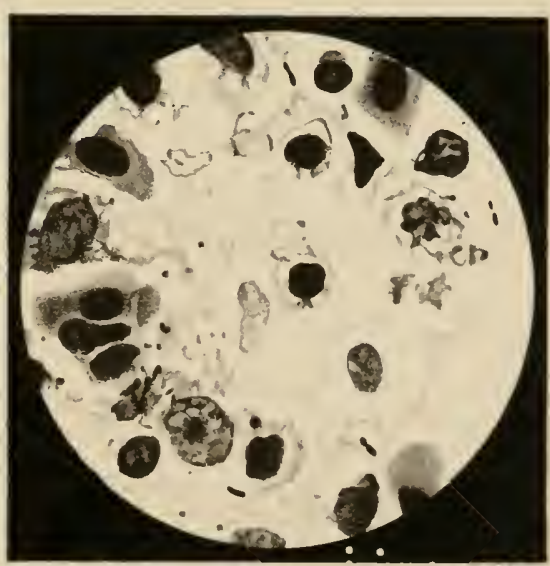

Fig. 42 




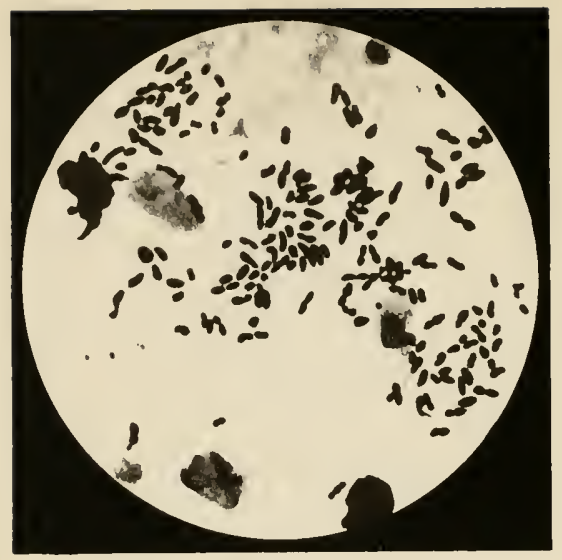

Fig. 43

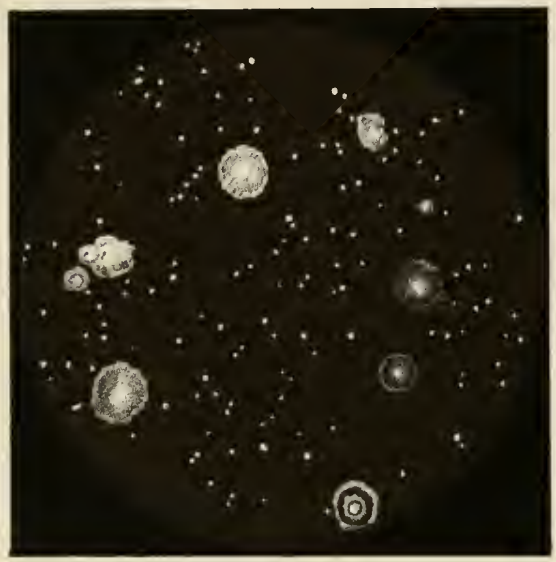

Fig. 45

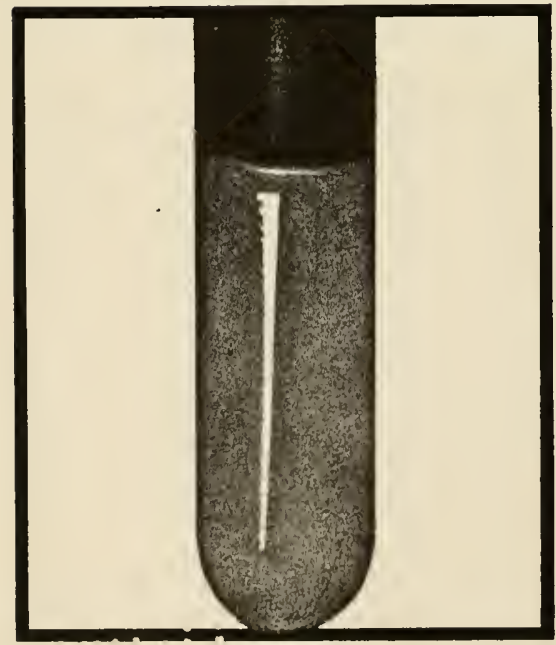

FIg. 47

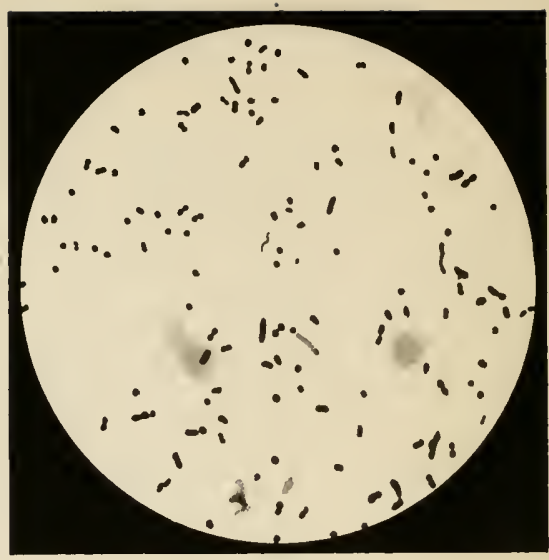

Fig. 44

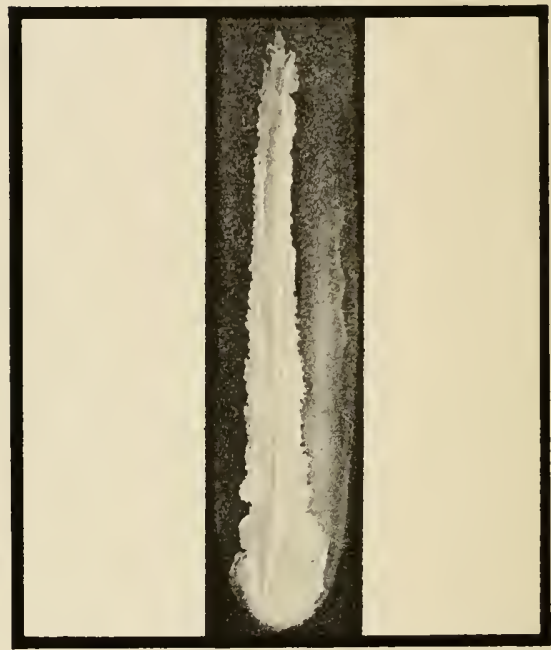

Fig. 46

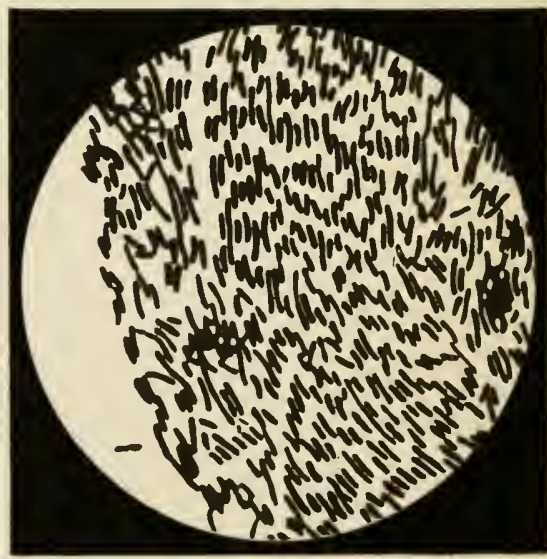

Fig. 48 
FIG. 43 .

Cover-glass specimen of the Intestinal Mucus of a Fowl dead of Fowl Enteritis. Magnifying power 1000.

The bacilli are present in great numbers and in pure culture.

FIG. 44.

Cover-glass specimen of the Intestinal Mucus of a similar Fowl as in Fig. 43. Magnifying power 1000.

FIG. 45 .

Plate cultivation in gelatine of the Bacillus of Fowl Enteritis. Natural size.

The small dots are colonies in the depth; the large patches are colonies on the surface of the gelatine. The difference between the plate cultivation of the bacillus of Enteritis and that of the bacillus of Fowl Cholera (Fig. 35) is obvious. Both plates were made at the same time, and were kept under precisely the same conditions.

FIG. 46.

Streak culture on the surface of gelatine of the Bacillus of Fowl Enteritis. Natural size.

FIG. 47 .

Stab-culture of same. Natural size.

F1G. 48.

Cover-glass specimen,-impression of a Superficial Colony from a plate culture, the margin of a Colony being shown here. Magnifying power 1000. 
Fig. 49 .

Cover-glass specimen from a culture on gelatine of the Bacillus of Fowl Enteritis. Magnifying power 1000.

$$
\text { FIG. } 5 \text { o. }
$$

Cover-glass specimen from an old broth culture of the Bacillus of Fowl Enteritis, showing involution forms and degenerating filaments of the Bacilli. - Magnifying power rooo.

\section{F1G. 5 I.}

Section through the Liver of a Fowl dead of the Fowl Enteritis. Magnifying power 1000 , showing numerous bacilli in the capillary blood-vessels, these latter being seen cut across.

\section{FIG. 52.}

Cover-glass specimen of an Agar culture of the Bacillus of "the Cramps" of Young Pheasants. Magnifying power 2000.

\section{FiG. 53 .}

Cover-glass specimen of a gelatine culture of the same Bacillus. Magnifying power 2000. The difference in the shape of the bacilli between those from an agar- and those from a gelatine culture is striking. 


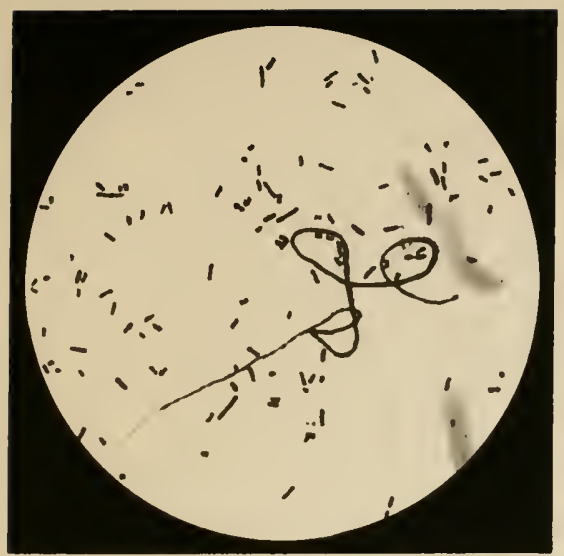

Fig. 49

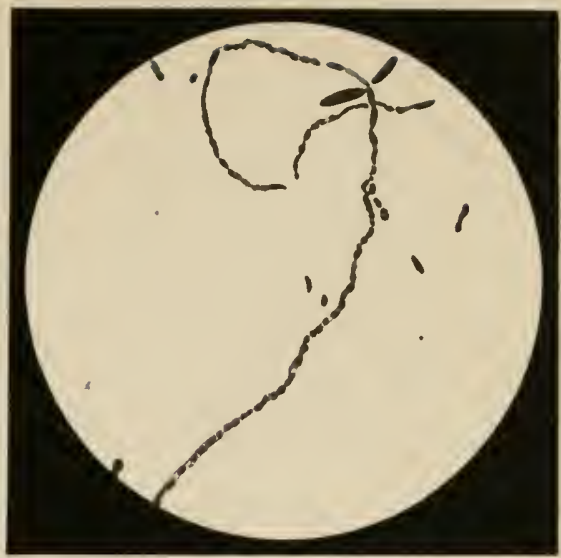

Fig. 50

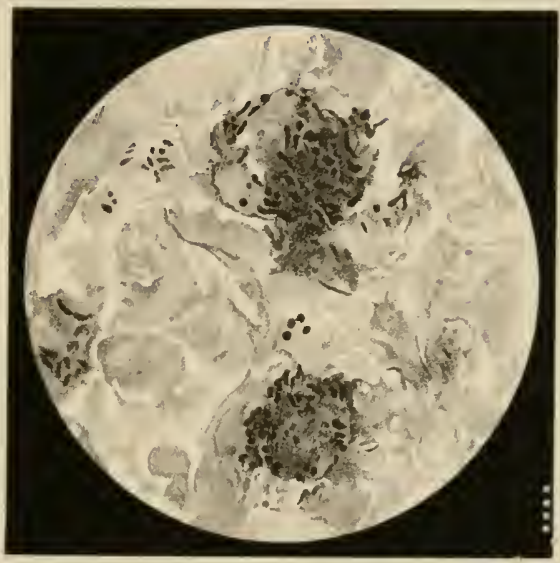

Fig. 51

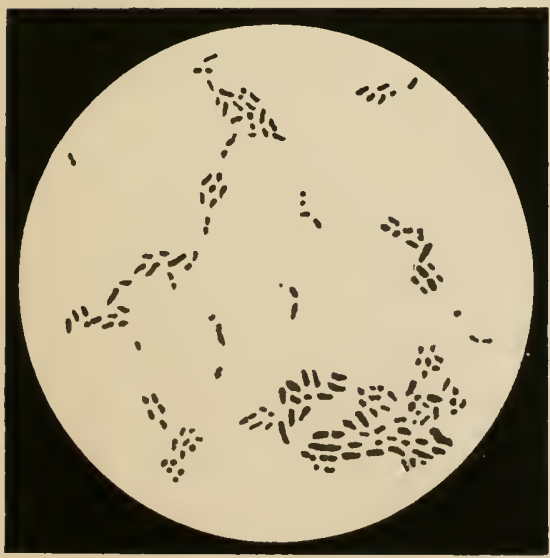

Fig. 52

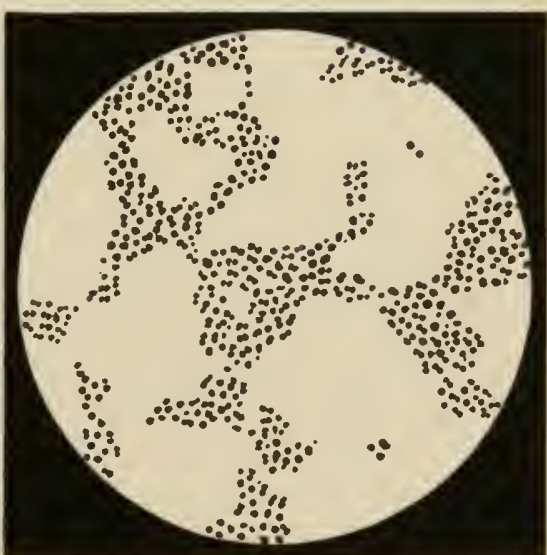

Fig. 58 



\title{
MESSRS. MACMILLAN \& CO.'S PUBLICATIONS.
}

\section{WORKS BY THE SAME AUTHOR.}

\author{
MICRO-ORGANISMS AND DISEASE. An Introduction into
} the Study of Specific Micro-Organisms. Illustrated. Third Edition, Revised. Crown Svo. 6s.

$A C A D E M Y$ :- "No present line of medical research is so likely to prove fraught with benefit of diminishing disease and spreading health as that to which Dr. Klein's little book offers an introduction, lucid and concise. . . . Dr. Klein's exposition is specially opportune as offering to the general public, in the few clear pages of an authority and expert, a short and safe way out of the tangle of diverse opinion. When, as herc, the authority of experience is modest, and skill is tentative and cautious, they have most weight. This book in a marked degree shows these to be the graces of its author as an observer and teacher, and the discovery increases one's previous sense of his trustworthiness."

SATURDAY REVIEW:- "By far the most important book among those relating to matters of health and disease which are now before us is that of Dr. Klein on Micro-Organisms. . . . Dr. Klein is well known as a first-rate observer and careful reasoner, and has, moreover, for many years given untiring study to the questions which he now handles. Ile is therefore fully qualified to give, as he here does, a succinct account of what is known regarding this difficult matter. . . . Dr. Klein's book, while nainly intended for the expert, is written with sufficient clearness to be of considerable interest to all who may wish to know what is being done regarding the important question of the germ-origin of disease."

\section{THE BACTERIA IN ASIATIC CHOLERA. Crown Swo. 5s.}

LANCET:- "No single observer has contributed more to the knowledge on this subject than Dr. Klein, and the work before us is a thoroughly scientific exposition of it in all its bearings . . a perusal of his book cannot fail to leave the impression that there is still much to be learnt concerning cholera and its relationship to bacterial life."

MIANCHESTER GUARDIAN:- "Dr. Klein is one of our best English bacteriologists, and in this book any one interested in the subject will find the clearest description of the delicate experiments made to prove the nature of the comma-bacilli. There is throughout the volume the tone that should pervade all scientific works, of candid acknowledgment of the work and opinions of opponents, and at the same time of determination to subject their conclusions to the most rigorous and searching tests."

VIENNA WEEKLY NEWS:- "Professor Klein's criticism, which is absolutely free from personalities, is based on the results of careful microscopical and lacteriological examinations carried on, for many years, in England and India. The book contains a full synopsis of the present state of the etiology of cholera, and being written in a clear style, will initiate the student into the study of bacteriology. It will, moreover, be a valuable guide to beginners by showing them how cautious one must be in drawing conclusions especially in bacteriological matters. By a number of excellent illustrations the publishers have added to the special features of the book, which is sure to obtain a large circulation in the medical world."

MACMILLAN AND CO., LONDON. 


\section{MIESSRS. MACMILLAN \& CO.'S BOOKS}

\section{ON BIOLOGY, ANATOMY, AND PHYSIOLOGY.}

\section{LESSONS IN ELEMENTARY PHYSIOLOGY. By THOMAS H.}

lluxtey, F.R.S. Illustrated. New Edition. Revised. 18mo. 4s.6d.

ELEMENTARY BIOLOGY. By T. H. HuxLEY, F.R.S., and H. N. MARTin, F.R.S. New Edition. Revised and extended by G. B. Howes and D. H. SCOTT. Crown 8ro. IOs. 6d.

AN ATLAS OF PRACTICAL ELEMENTARY BIOLOGY. By G. I3. Howes. With a I'reface by T. II. Huxleey, F.R.S. 4to. I4s.

COMPARATIVE EMIBRYOLOGY (A Treatise on). By F. M. BALFOUR, M.A., F.R.S. Illustrated. In 2 Vols. Vol. I., 18s.; Vul. II., 2 Is.

THE ELEMENTS OF EMBRYOLOGY. By Professor M. Foster, M.D., F.R.S., and the late Francis M. BALFOUR, F.R.S. Third Edition, Revised. Edited by AdAM SEIGWick, M.A., and WAlTer HeApe. IOs. 6d.

A TEXT-BOOK OF COMPARATIVE ANATOMY. By Dr. ARnOLD Lang, Professor of Zoology in the University of Zurich, formerly Ritter Professor of Phylogeny in the University of Jena. With Preface to the Englisis Translation by Prof. Dr. ERnst IIAEcker, Director of the Zoological Institute in Jena. Translated into English by Henry M. Bernard, M.A. Cantab., and Matilda Bernard. Part I. Svo. I $7 \mathrm{~s}$. net.

TEXT-BOOK OF PHYSiOlogy. By Professor Michael Foster, MI.D., F.R.S. With lllustrations. Sixth Edition, largely revised. In Three Parts. Svo. Parts I. and II., IOs. 6d. each. Part III., 7s. 6d. Part IV., Ios. 6d.

PRACTICAL PHYSIOLOGY AND HISTOLOGY: An Elementary Course. By Professor M. Foster, F.R.S., and J. N. Langley, F.R.S. Crown 8vo. 7s. 6d.

ZOOTOMY (Vertebrata): A Course of Instruction in. By Professor T. J. PARKER. Illustrated, Crown Svo. Ss. 6d.

ELEMENTARY LESSONS IN ANATOMY. By St. George MIVART, F.R.S. With 400 Illustrations. I8mo. 6s. 6d.

ELEMENTS OF COMPARATIVE ANATOMY OF VERTEBRATES. Adapted from the German of ROBERT Wiedersieim. By Professor IV. N. PARKer. With 270 Illustrations. Svo. 125. 6d.

THE OSTEOLOGY OF THE MAMMALIA (An Introduction to). by Willaam Henry Flower, LL.D., F.R.S. Third Edition. Revised with the assistance of Hans Gavow, Ph.D., M.A. IOs. 6d.

THE MYOLOGY OF THE RAVEN (Corvus corax sinuatus). A Guide to the Study of the Muscular System in Birds. By R. W. Shufeldt. Illustrated. 8vo. I3s, net.

ELEMENTARY PRACTICAL HISTOLOGY. By WILLIAM FEARNLEY. With Illustrations. Crown 8vo. 7s. 6d.

LESSONS IN ELEMENTARY BIOLOGY. By Professor $\mathrm{T}$. JefFrey PARKer, B.Sc., F.K.S. Illustrated. Crown 8vo. ros. 6d.

UISEASES OF FIELD AND GARDEN CROPS, chiefly such as are caused by Fungi. By Worthington G. Smith, F.L.S. Fcap. 8vo. 4s. 6d.

TIMBER AND SOME OF ITS DISEASES. By H. MARSHALL WAR1, F.K.S., Professor of Thotany Royal Indian Engineering Cullege, Cooper's IIill. Cr. Svo. 6s. 


\section{MESSRS. MACMILLAN \& CO.'S BOOKS.}

\section{BIOLOGY.}

HANDBOOK OF FIELD AND GENERAL ORNITHOLOGY. By Prof. Elliott Covis, M.A. Illustrated. Sro. Ios, net.

ORGANIC EVOLUTION as the Result of the Inheritance of Acquired Characters, according to the Laws of Organic Growth. By Dr. G. II. T. Einer. Translated loy J. T. Cunnisgiiam, F.R.S.E. Sro. I2s. 6d.

Works by ALFRED RUSSEL WALLACE, LL.D.

DARIVINISMI: An Exposition of the Theory of Natural Selection. 3d Edition. Crown Sro. 9s.

NATURAL SELECTION: AND TROPICAL NATURE. New Edition. Crown Svo. 6 .

ISLAND LIFE. New Ed. Crown Svo. 6 s.

Works by the Right Hon. Sir John LubBock, F.R.S., D.C.L.

THE ORIGIN AND METAMORPHOSES OF INSECTS. Illus. Crown Svo. 3s. 6d.

ON BRITISH WILD FLOWERS CONSIDERED IN RELATION TO INSECTS. Illustrated. Crown Svo. 4s. 6d.

FLOWERS, FRUITS, AND LEAVES, Illustrated, 2d Edition. Crown Svo. 4s. 6d.

\section{BOTANY.}

PHYSIOLOGICAL BOTANY. I. Outlines of the Histology of Phænogamous Plants. II. Vegetable Physiology. By George Lincoly Goodale, M.A., M.D., Professor of Botany in Harvard University. Svo. IOs. 6d.

STRUCTURAL BOTANY, OR ORGANOGRAPHY ON THE BASIS OF MORPHOLOGY. To which are added the Principles of Taxonomy and Phytography, and a Glossary of Botanical Terms. By Prof. Asa Gray, LL.D. Svo. Ios. 6d.

FIRST LESSONS IN PRACTICAL BOTANY. By G. T. BETTANY. I $8 \mathrm{mo}$. Is.

A COURSE OF PRACTICAL INSTRUCTION IN BOTANY. By F. O. Bower, D.Sc., F.R.S., Regius Professor of lotany in the University of Glasgow. Crown Sro. IOs. 6d. Abridged Edition.

[In prefaration.

THE FERTILISATION OF FLOWERS. By HERMANN MÜLLER. Translated by D'ArCy W. Thompson, B.A., Professor of Biology in University College, Dundee. Preface by C. DARw1s, F.R.S. Illustrated. Svo. 21 s.

Works by DANIEL Oliver, F.R.S.

Late Professor of Botany in University College, London.

LESSONS IN ELEMENTARY BOTANY. Illustrated. Fcap. 8vo. 4s. 6d.

FIRST BOOK OF INDIAN BOTANY. Illustrated. Extra fcap. 8vo. 6s. 6d.

Works by Sir JoserH HOOKER, F.R.S., \&c.

PRIMER OF BOTANY. Illustrated. I 8 mo. IS.

THE STUDENT'S FLORA OF THE BRITISH ISLANDS. 3d Edition, revised. Globe Sro. Ios. 6d. 


\section{MESSRS. MACMILLAN \& CO.'S PUBLICATIONS.}

\section{BOOKS ON MEDICINE.}

A MANUAL OF PUBliC IIEALTh. By A. Wynter Blyti, M.R.C.S. 8vo. i7s. net.

LESSONS ON PRESCRIPTIONS AND THE ART OF PRESCRIBING. By W. H. Griffiths. Adapted to the Pharmacopoia, 1885 . 18mo. 3s. 6 d.

A TEXT-BOOK OF PATHOLOGY, SYSTENATIC AND PRACTICAL, By D. J. Hamilton, F.R.S.E., Professor of Pathological Anatomy, University of Aberdeen. Illustrated. Vol. 1. 8vo. 25s.

A TEXT-BOOK OF GENERAL THERAPEUTICS. By W. IIAle White, NI.D., Senior Assistant Physician to and Lecturer in Materia Medica at Guy's Hospital. Illustrated. Cr. 8 vo. $8 \mathrm{~s}, 6 \mathrm{~d}$.

TEXT-BOOK OF PATIOLOGICAL ANATONY AND PATIOGENESIS. By Prof. E. Ziegler. Translated and Edited by Donald Macalister, M.A., M.D. Fellow and Medical Lecturer of St. John's College, Cambridge. Illustrated. $8 \mathrm{vo}$.

Part I.-GENERAL PATHOLOGICAL ANATOMY. 2d Ed. I2s. 6d. Part II.-SPECIAL PATHOLOGICAL ANATOMY. Sections I.-VIII. 2d Edition. 12s. 6d. Sections IX.-XII. 12s. 6d.

Works by T. LAUDER BRUNTON, M.D., F.R.S., Examiner in Materia Medica in the University of London, in the Victoria University, and in the Royal College of Physicians, London.

A TEXT-BOOK OF PHARMACOLOGY, THERAPEUTICS, AND MATERIA MEDICA. Adapted to the United States Pharmacopœia, by F. H. Williass, M.D., Boston, Mass. 3d Edition. Adapted to the New British Pharmacopeia, 1885, and additions, 18gr. 8vo. 2 rs. Or in 2 Vols. 22s. 6d.

TABLES OF MATERIA MEDICA; A Companion to the Materia Medica Museum. Illustrated. Cheaper Issue. 8vo. 5s.

\section{THE CROONIAN LECTURES FOR I8S9.}

THE INTRODUCTION TO MODERN TIIERAPEUTICS. Being the Croonian Lectures in the relationship between Chemical Structure and Physiological Action delivered before the Royal College of Physicians in London, June 1889. By T. LAUDER Brunton, M.D., D.Sc. Edin., LL.D. A berd., F.R.C.P., F.R.S. 8vo, cloth, 3s. 6d. net.

DISORDERS OF DIGESTION: THEIR CONSEQUENCES AND TREATMENT. By T. LAUdER BRUNTON, M.D. This work contains, in addition to the Lettsomian Lectures, a number of other Papers by the Author on similar subjects. 8vo, 1os. $6 \mathrm{~d}$. EDINBURGH MEDICAL JOUR 'AL.--"Distinguished by accurate observation and original thinking, these papers have been largely instrumental in moulding current medical opinion in regard to many of the subjects with which they are concerned."

LONDON MEDICAL RECORD.- "We can cordially recommend the volume, as one which combines much practical and technical information and sound common sense."

$$
\text { MACMILLAN AND CO, LONDON. }
$$


.1 
THIS BOOK IS DUE ON THE LAST DATE STAMPED BELOW

AN INITIAL FINE OF 25 CENTS WILL BE ASSESSED FOR FAILURE TO RETURN THIS BOOK ON THE DATE DUE. THE PENALTY WILL INCREASE TO 50 CENTS ON THE FOURTH DAY AND TO $\$ 1.00$ ON THE SEVENTH DAY OVERDUE.

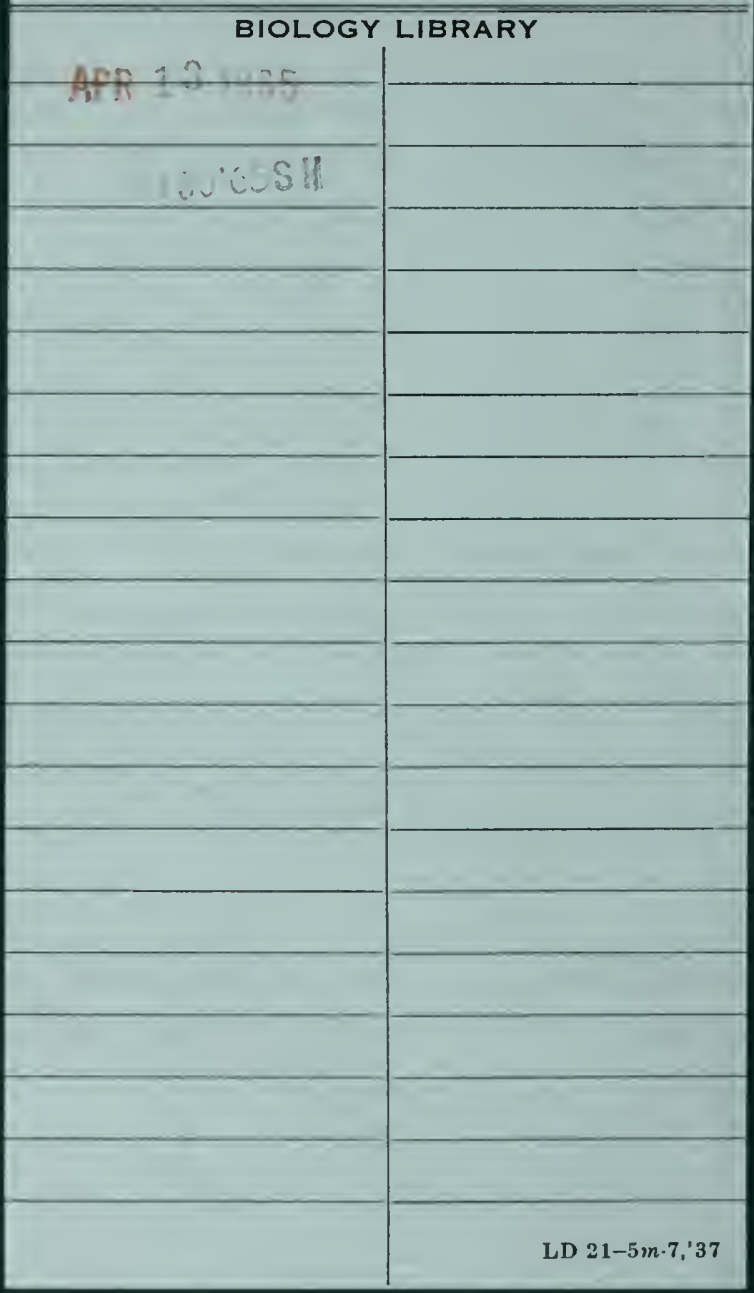




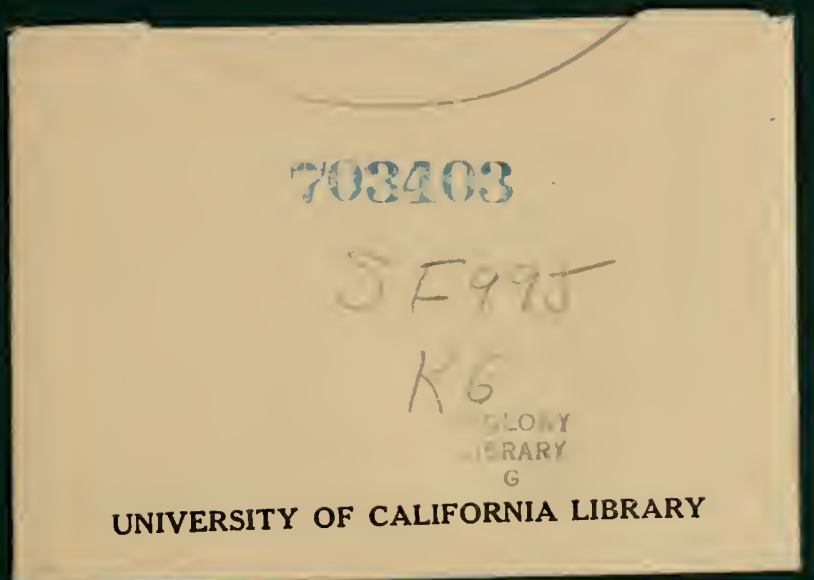




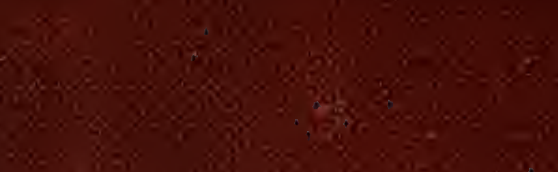

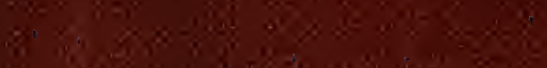

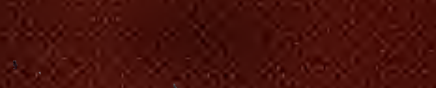

7.6.

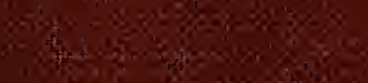

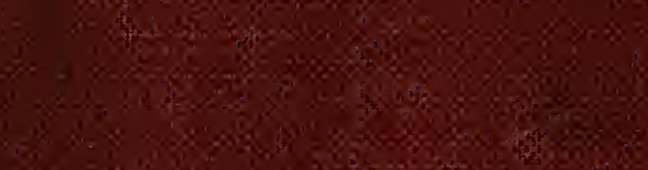

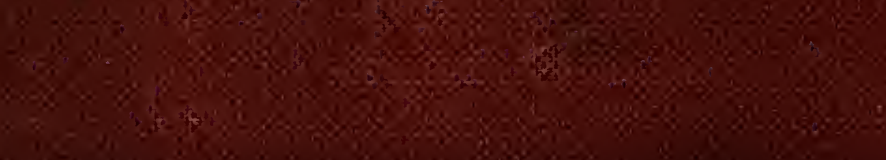

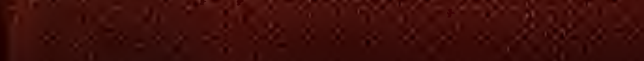

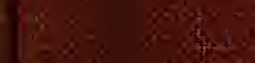

